\title{
Sensitivity Analysis of Thermoacoustic Instability with Adjoint Helmholtz Solvers
}

\author{
Matthew P. Juniper* \\ Engineering Department, University of Cambridge, CB2 1PZ, U.K.
}

(Dated: November 6, 2018) 


\begin{abstract}
Gas turbines and rocket engines sometimes suffer from violent oscillations caused by feedback between acoustic waves and flames in the combustion chamber. These are known as thermoacoustic oscillations and they often occur late in the design process. Their elimination usually requires expensive tests and re-design. Full scale tests and laboratory scale experiments show that these oscillations can usually be stabilized by making small changes to the system. The complication is that, while there is often just one unstable natural oscillation (eigenmode), there are very many possible changes to the system. The challenge is to identify the optimal change systematically, cheaply, and accurately. This paper shows how to evaluate the sensitivities of a thermoacoustic eigenmode to all possible system changes with a single calculation by applying adjoint methods to a thermoacoustic Helmholtz solver. These sensitivities are calculated here with finite difference and finite element methods, in the weak form and the strong form, with the discrete adjoint and the continuous adjoint, and with a Newton method applied to a nonlinear eigenvalue problem and an iterative method applied to a linear eigenvalue problem. This is the first detailed comparison of adjoint methods applied to thermoacoustic Helmholtz solvers. Matlab codes are provided for all methods and all figures so that the techniques can be easily applied and tested. This paper explains why the finite difference of the strong form equations with replacement boundary conditions should be avoided and why all of the other methods work well. Of the other methods, the discrete adjoint of the weak form equations is the easiest method to implement; it can use any discretization and the boundary conditions are straightforward. The continuous adjoint is relatively easy to implement but requires careful attention to boundary conditions. The Summation by Parts finite difference of the strong form equations with a Simultaneous Approximation Term for the boundary conditions (SBP-SAT) is more challenging to implement, particularly at high order or on non-uniform grids. Physical interpretation of these results shows that the well-known Rayleigh criterion should be revised for a linear analysis. This criterion states that thermoacoustic oscillations will grow if heat release rate oscillations are sufficiently in phase with pressure oscillations. In fact, the criterion should contain the adjoint pressure rather than the pressure. In self-adjoint systems the two are equivalent. In non-self-adjoint systems, such as all but a special case of thermoacoustic systems, the two are different. Finally, the sensitivities of the growth rate of oscillations to placement of a hot or cold mesh are calculated, simply by multiplying the feedback sensitivities by a number. These sensitivities are compared successfully with experimental results. With the same technique,
\end{abstract}


the influence of the viscous and thermal acoustic boundary layers is found to be negligible, while the influence of a Helmholtz resonator is found, as expected, to be considerable.

\footnotetext{
*mpj1001@cam.ac.uk; http://www2.eng.cam.ac.uk/〜mpj1001/MJ_biography.html
} 


\section{INTRODUCTION}

Rockets and jet engines have unrivalled power to weight ratios and are therefore ideal for aerospace applications. They are designed to operate in steady flow, in the sense that the time average flow is the same as the ensemble average flow. Sometimes, however, heat release rate oscillations lock into acoustic oscillations so violently that they cause excessive heat transfer, structural vibration, and even catastrophic failure [2]. These are known as thermoacoustic oscillations.

The thermoacoustic mechanism is similar to that of a piston engine but with an acoustic wave taking the place of the piston: when more heat release rate occurs during moments of high pressure, and less heat release rate occurs during moments of low pressure, more work is done during the expansion phase of the acoustic cycle than is absorbed during the compression phase, causing oscillations to grow [3]. A complete analysis can be found in [4] who writes (page 226) that "if $m^{\prime}$ [mass injection] is in phase with $p^{\prime}$ [acoustic pressure], or $F^{\prime}$ [momentum injection] with $u^{\prime}$ [acoustic velocity] or $Q^{\prime}$ [fluctuating heat release rate] with $T^{\prime}$ [acoustic temperature] then energy will be continuously fed into the disturbance". This quote will be re-examined in $\S$ VIII in light of the results in this paper.

A key component in this mechanism is the heat release rate's response to acoustic perturbations. As the ratio of air to fuel in gas turbines is increased in order to reduce nitrous oxide emissions, flames tend to become more responsive to acoustics [2]. As a consequence, thermoacoustic oscillations are increasingly problematic for gas turbines. Worse still, their behavior is exceedingly sensitive to small parameter or shape changes [5]. Oscillations therefore tend to re-appear during the later stages of testing and are rarely predicted reliably by component tests and analysis [6-8].

The goal of rocket and gas turbine manufacturers is to design an engine that is linearly stable to thermoacoustic oscillations over the entire operating regime. Currently, this is achieved through (i) extensive testing, which is expensive, (ii) the avoidance of certain engine operating regimes, which reduces flexibility, and (iii) the retro-fitting of passive dampers such as Helmholtz resonators, which add weight and modes of failure [7, 9]. A systematic method to identify optimal stabilization strategies will speed up development, eliminate dangerous operating points, and either determine the optimal placement of passive dampers or remove the need for them entirely. Such a method can exploit three convenient facts: firstly, the 
requirement is for linear (rather than nonlinear) stability, meaning that the tools of linear analysis can be used; secondly, no more than a handful of thermoacoustic modes are ever unstable; thirdly, usually many parameters of the system can be altered. The challenge is therefore to identify the most influential parameters for each mode and how they should be changed optimally to stabilize the system.

An ideal solution is to develop a faithful model of the thermoacoustic system's linear behavior and then to combine linear stability analysis [10] with adjoint methods [11]. The stability analysis identifies the handful of unstable eigenvalues. The adjoint methods then show, in a single calculation for each eigenvalue, how each eigenvalue is affected by every parameter in the system. While this sensitivity could be calculated with finite difference calculations, this would require a single calculation for each parameter, which is too expensive. This gradient information enables efficient optimization through, for example, change of boundary conditions, change of flame shape, change of geometry, or optimal addition of feedback devices such as Helmholtz resonators.

Different families of linear thermoacoustic models exist in the literature: (i) Galerkin methods, in which the acoustic perturbation is projected onto a basis set, which is usually the pure acoustic modes of the system; (ii) travelling wave methods (known as network models), in which the acoustics are expressed as travelling waves; (iii) Helmholtz methods, in which the Helmholtz equation with heat release is solved in the frequency domain using finite difference, finite volume, or finite element for spatial discretization. The Galerkin method is easy to write in adjoint form [12, 13] but has convergence problems [14] and is not straightforward to apply in complex geometries. The travelling wave method is relatively easy to write in adjoint form [5, 15] but is restricted to simple acoustics. Nevertheless, this method is extensively used in industry and is a promising route to early application of adjoint methods in thermoacoustics. For example, adjoint-based optimization is applied in [16] to stabilize all the modes of a travelling wave model of a laboratory burner and in [17] to stabilize all the modes of a travelling wave model of an annular combustor, using the adjoint version of an industrial code, LOTAN $[18,19]$.

This paper shows how to derive the adjoints for Helmholtz solvers with finite difference and finite element discretizations, in the weak form and the strong form, with different boundary implementations, with the discrete adjoint and the continuous adjoint, and with a Newton method applied to a nonlinear eigenvalue problem and an iterative method applied 
to a linear eigenvalue problem. The methods are described in one spatial dimension but the principles scale readily to $2 \mathrm{D}$ and $3 \mathrm{D}$.

The work reported in this paper started at the Stanford Center for Turbulence Research Summer Program in 2014. The Program's proceedings [20] contain an outline of the mathematical principles required for adjoint Helmholtz solvers with active iteration, but no numerical implementation or results. Since then there have been some applications of adjoint Helmholtz solvers in the literature: (i) to optimize acoustic damper placement [21]; (ii) to speed up uncertainty quantification [22, 23], and (iii) to investigate the effects of asymmetry in annular combustors [24]. Adjoints have been applied (in collaboration with the author) to the three dimensional finite volume-based thermoacoustic solver AVSP [25, Ch. 7]. The supplementary materials of [5] contain rudimentary direct and adjoint codes for a Galerkin method, a travelling wave method, a finite difference Helmholtz solver, and a finite element Helmholtz solver in order to demonstrate extreme sensitivity in thermoacoustics. The codes in [5] contain only Dirichlet boundary conditions, the discrete adjoint, and the Newton method. The current paper is the first paper on thermoacoustic Helmholtz solvers containing (i) the Summation by Parts Finite Difference formulation with a Simultaneous Approximation Term (SBP-SAT); (ii) Neumann and frequency-dependent Robin boundary conditions for all types of discretizations; (iii) details of the numerical methods; (iv) comparisons of all methods and explanations of the sources of numerical problems; (v) physical interpretations of the base state sensitivities, structural sensitivities, and receptivities; (vi) the conclusion that Rayleigh's criterion needs to be revised for non-self-adjoint systems; (vii) optimization using base state sensitivities; (viii) estimation of the influence of neglected phenomena using feedback sensitivities, and (ix) estimation of the influence of retro-fitted components using feedback sensitivities. The presentation is pedagogical and contains numerical details that the experienced reader can skip. Matlab codes are provided for the four discretizations, all boundary conditions, both types of adjoint, and both types of solution methods so that the reader can apply the techniques easily and experience first hand some of the benefits and pitfalls of the different approaches. 


\section{MODEL}

\section{A. The governing equations for mass, momentum, and energy}

In this section all equations are dimensional and are written in terms of pressure, $p$, density, $\rho$, temperature, $T$, entropy, $\varsigma$, and velocity $\underline{\mathbf{u}}$. Each total quantity ô is comprised of a mean $\bar{o}$ and an acoustic fluctuation õ. Following [1], the behaviour of a single component gas is modelled by the equation of state, the definition of entropy for a perfect gas, and transport equations for mass, momentum, and entropy of the total quantities:

$$
\begin{aligned}
\frac{\hat{p}}{\hat{\rho}} & =r_{g} \hat{T} \\
\hat{\varsigma}-\varsigma_{r e f} & =c_{p} \ln \frac{\hat{T}}{T_{r e f}}-r_{g} \ln \frac{\hat{p}}{p_{\text {ref }}} \\
\frac{\mathrm{D} \hat{\rho}}{\mathrm{D} t} & =-\hat{\rho} \nabla \cdot \underline{\hat{\mathbf{u}}} \\
\hat{\rho} \frac{\mathrm{D} \underline{\hat{\mathbf{u}}}}{\mathrm{D} t} & =-\nabla \hat{p} \\
\hat{\mathrm{D}} \hat{\mathrm{\varsigma}} & =\frac{r_{g}}{\mathrm{D} t} \hat{\hat{p}}
\end{aligned}
$$

where $c_{p}$ is the specific heat capacity at constant pressure, $r_{g}$ is the specific gas constant, and $\hat{\dot{q}_{h}}$ is the heat release rate per unit volume, which is non-zero over some region $h(\underline{\mathbf{x}})$. Small amplitude acoustic fluctuations $(\underline{\tilde{\mathbf{u}}}, \tilde{p}, \tilde{\rho}, \tilde{\varsigma}, \tilde{T})$ are considered, superimposed onto a zero Mach number steady base flow $(\bar{p}, \bar{\rho}, \bar{\varsigma}, \bar{T})$. Following [4, section III], the acoustic equations are further perturbed by adding fluctuating mass injection per unit volume per unit time, $\epsilon \delta \tilde{\dot{m}}$, fluctuating body force per unit volume, $\epsilon \delta \tilde{\mathbf{f}}$, and fluctuating heat release rate per unit volume per unit time, $\epsilon \delta \tilde{\dot{q}}$. These perturbations, when suitably combined, can describe any physical perturbation to the system $(\S \mathrm{VI})$. The linearized equations for the acoustic fluctuations are:

$$
\begin{aligned}
& \frac{\tilde{p}}{\bar{p}}-\frac{\tilde{\rho}}{\bar{\rho}}-\frac{\tilde{T}}{\bar{T}}=0 \\
& \tilde{\varsigma}=c_{p} \frac{\tilde{\bar{T}}}{\bar{T}}-r_{g} \frac{\tilde{\bar{p}}}{\bar{p}} \\
& \frac{\partial \tilde{\rho}}{\partial t}+\underline{\tilde{\mathbf{u}}} \cdot \nabla \bar{\rho}+\bar{\rho} \nabla \cdot \underline{\tilde{\mathbf{u}}}=0+\epsilon \delta \tilde{\dot{m}} \\
& \bar{\rho} \frac{\partial \underline{\tilde{\mathbf{u}}}}{\partial t}+\nabla \tilde{p}=0+\epsilon \delta \tilde{\mathbf{f}} \\
& \frac{\partial \tilde{\varsigma}}{\partial t}+\underline{\tilde{\mathbf{u}}} \cdot \nabla \bar{\varsigma}=\frac{r_{g}}{\bar{p}}\left(\tilde{\dot{q}_{h}}+\epsilon \delta \tilde{\dot{q}}\right) .
\end{aligned}
$$


If the gas is perfect then (1b) implies that $\nabla\left(\ln \bar{\rho}+\bar{\varsigma} / c_{p}\right)=(\nabla \ln \bar{p}) / \gamma$, which is zero because $\bar{p}$ is uniform because the flow has zero Mach number. Combining this with (2a) and (2b) and substituting into (2c) divided by $\bar{\rho}$ plus (2e) divided by $c_{p}$ gives the acoustic equations for mass/energy (3b) and momentum (2d, 3a):

$$
\begin{gathered}
\frac{\partial \underline{\underline{\mathbf{u}}}}{\partial t}+\frac{1}{\bar{\rho}} \nabla \tilde{p}=\frac{\epsilon \delta \tilde{\mathbf{f}}}{\bar{\rho}} \\
\frac{1}{\gamma} \frac{\partial}{\partial t}\left(\frac{\tilde{p}}{\bar{p}}\right)+\nabla \cdot \underline{\tilde{\mathbf{u}}}=\frac{\epsilon \delta \tilde{\dot{m}}}{\bar{\rho}}+\frac{(\gamma-1)}{\gamma}\left(\frac{\tilde{\tilde{q}}}{\bar{p}}+\frac{\epsilon \delta \tilde{\dot{q}}}{\bar{p}}\right) .
\end{gathered}
$$

\section{B. The heat release model}

The heat release model is a distributed $n-\tau$ model adapted from [1]. The local heat release rate perturbation per unit volume, $\tilde{q}_{h}(\underline{\mathbf{x}}, t)$, is proportional to the velocity, $\underline{\tilde{\mathbf{u}}}$, in a reference direction, $\underline{\hat{\mathbf{w}}}$, integrated over a measurement region, $w(\underline{\mathbf{x}})$, some time $\tau(\underline{\mathbf{x}})$ earlier:

$$
\tilde{q}_{h}(\underline{\mathbf{x}}, t)=\eta h(\underline{\mathbf{x}}) \int_{\Omega} w(\underline{\underline{\mathbf{x}}}) \underline{\hat{\mathbf{w}}} \cdot \underline{\tilde{\mathbf{u}}}(\underline{\breve{\mathbf{x}}}, t-\tau(\underline{\mathbf{x}})) \mathrm{d} \underline{\underline{\mathbf{x}}},
$$

where $h(\underline{\mathbf{x}})$ is a distribution of heat release in space such that $\int_{\Omega} h(\underline{\mathbf{x}}) \mathrm{d} \underline{\mathbf{x}}=1$, and $w(\underline{\mathbf{x}})$ is a distribution of measurement in space such that $\int_{\Omega} w(\underline{\mathbf{x}}) \mathrm{d} \underline{\mathbf{x}}=1$. The integral $\int_{\Omega} \tilde{\tilde{q}_{h}}(\underline{\mathbf{x}}) \mathrm{d} \underline{\mathbf{x}}$ has units $J s^{-1}$ and the velocity $\underline{\tilde{u}}$ has units $m s^{-1}$, so the constant $\eta$ has units $\mathrm{Jm}^{-1}$.

It is useful to express $\eta$ in terms of the magnitude of the Flame Transfer Function (FTF). If $w(\underline{\mathbf{x}})$ is set to a Dirac delta function at $\underline{\mathbf{x}}_{w}$ and $\tau(\underline{\mathbf{x}})$ is uniform in space and $\tilde{u}_{w} \equiv \underline{\hat{\mathbf{w}}} \cdot \underline{\tilde{\mathbf{u}}}$ then (4) becomes $\int_{\Omega} \tilde{q}_{h}(\underline{\mathbf{x}}, t) \mathrm{d} \underline{\mathbf{x}}=\eta \tilde{u}_{w}\left(\underline{\mathbf{x}}_{w}, t-\tau\right)$. The FTF is non-dimensional and has magnitude $|\mathrm{FTF}|=\left|\left(\int_{\Omega} \tilde{\dot{q}}_{h} \mathrm{~d} \underline{\mathbf{x}}\right) / \overline{\dot{Q}_{h}}\right| /\left|\tilde{u}_{w}\left(x_{w}\right) / \bar{u}\right|$ where $\overline{\dot{Q}_{h}} \equiv \int_{\Omega} \overline{\dot{q}_{h}} \mathrm{~d} \underline{\mathbf{x}}$ is the mean heat release rate. Therefore

$$
|\eta|=\frac{\left|\int_{\Omega} \tilde{\dot{q}_{h}} \mathrm{~d} \underline{\mathbf{x}}\right|}{\left|\tilde{u}_{w}\left(\underline{\mathbf{x}}_{w}\right)\right|}=|\mathrm{FTF}| \frac{\overline{\dot{Q}_{h}}}{\bar{u}} \quad \text { with units } \mathrm{Jm}^{-1} \text {, as expected. }
$$

\section{Non-dimensionalization}

For dimensional consistency between the base state sensitivity $(\S \mathrm{V})$ and the feedback sensitivity (§VI), three new variables are defined:

$$
\tilde{\dot{m}}_{\bar{\rho}} \equiv \frac{\tilde{\dot{m}}}{\bar{\rho}}, \quad \tilde{\mathbf{f}}_{\bar{\rho}} \equiv \frac{\tilde{\mathbf{f}}}{\bar{\rho}}, \quad \tilde{\dot{q}}_{\bar{p}} \equiv \frac{\tilde{\dot{q}}}{\bar{p}} .
$$


The velocity $\underline{\tilde{\mathbf{u}}}$ is eliminated by subtracting the divergence of (3a) from the time derivative of $(3 \mathrm{~b})$. The specific volume is defined as $\bar{v} \equiv \bar{\rho}^{-1}$ and the thermal expansion factor as $\zeta \equiv(\gamma-1) / \gamma$. The equations are non-dimensionalized with the length of the combustion chamber, $L_{c}$, the ambient pressure, $p_{a}$, and the speed of sound in the ambient fluid, $\left(\gamma p_{a} / \rho_{a}\right)^{1 / 2}$. The pressure is uniform, so $\bar{p}=p_{a}$ throughout. The reference density is $p_{a} / c_{a}^{2}$, which means that the non-dimensional ambient density is $\gamma$. The non-dimensional governing equation is:

$$
\begin{aligned}
\frac{1}{\gamma} \frac{\partial^{2} \tilde{p}^{\star}}{\partial t^{2^{\star}}}-\nabla^{\star} \cdot\left(\bar{v}^{\star} \nabla^{\star} \tilde{p}^{\star}\right) & =\frac{\partial}{\partial t^{\star}} \epsilon \delta \tilde{\dot{m}}_{\bar{\rho}}^{\star}-\nabla^{\star} \cdot \epsilon \delta \tilde{\mathbf{f}}_{\bar{\rho}}^{\star}+\zeta\left(\frac{\partial}{\partial t^{\star}} \frac{\tilde{q}_{h}^{\star}}{\bar{p}^{\star}}+\frac{\partial}{\partial t^{\star}} \epsilon \delta \tilde{\dot{q}}_{\bar{p}}^{\star}\right) \\
\text { where } \frac{\tilde{q}_{h}^{\star}}{\bar{p}^{\star}} & =\frac{\eta^{\star}}{\bar{p}^{\star}} h^{\star}\left(\underline{\mathbf{x}}^{\star}\right) \int_{\Omega} w^{\star}\left(\underline{\breve{x}}^{\star}\right) \underline{\tilde{\mathbf{u}}}^{\star}\left(\underline{\breve{\mathbf{x}}}^{\star}, t^{\star}-\tau^{\star}\left(\underline{\mathbf{x}}^{\star}\right)\right) \mathrm{d} \underline{\underline{\mathbf{x}}}^{\star}
\end{aligned}
$$

\section{Reduction to one dimension}

The analysis in the rest of this paper is performed in one spatial dimension on $x \in[0,1]$. The results are compared with experiments on a Rijke tube with cross-sectional area $S_{c}$. All properties are assumed to be uniform over the cross-section. In (4), the heat release rate distribution in $3 \mathrm{D}$ space, $h(\underline{\mathbf{x}})$, is replaced with $h(x) / S_{c}$, where $h(x)$ is the heat release

rate distribution in $1 \mathrm{D}$ space, defined such that $\int_{0}^{1} h(x) \mathrm{d} x=1$. The $1 \mathrm{D}$ non-dimensional governing equation is:

$$
\begin{aligned}
\frac{1}{\gamma} \frac{\partial^{2} \tilde{p}^{\star}}{\partial t^{\star}}-\frac{\mathrm{d}}{\mathrm{d} x^{\star}}\left(\bar{v}^{\star} \frac{\mathrm{d} \tilde{p}^{\star}}{\mathrm{d} x^{\star}}\right) & =\frac{\partial}{\partial t^{\star}} \epsilon \delta \dot{m}_{\bar{\rho}}^{\star}-\frac{\mathrm{d}}{\mathrm{d} x^{\star}} \epsilon \delta \tilde{\mathbf{f}}_{\bar{\rho}}^{\star}+\zeta\left(\frac{\partial}{\partial t^{\star}} \frac{\tilde{\dot{q}}_{h}^{\star}}{\bar{p}^{\star}}+\frac{\partial}{\partial t^{\star}} \epsilon \delta \tilde{\dot{q}}_{\bar{p}}^{\star}\right) \\
\text { where } \frac{\tilde{q}_{h}^{\star}}{\bar{p}^{\star}} & =\frac{\eta^{\star}}{\bar{p}^{\star} S_{c}^{\star}} h^{\star}\left(x^{\star}\right) \int_{0}^{1} w^{\star}\left(\breve{x}^{\star}\right) \tilde{u}^{\star}\left(\breve{x}^{\star}, t^{\star}-\tau^{\star}\left(x^{\star}\right)\right) \mathrm{d} \breve{x}^{\star} .
\end{aligned}
$$

A non-dimensional heat release factor is then defined as $n^{\star} \equiv \eta^{\star} /\left(\bar{p}^{\star} S_{c}^{\star}\right)$.

\section{E. Modal decomposition to the frequency domain}

The ${ }^{\star}$ that designated nondimensional variables is now dropped and a modal decomposition is performed by substituting $\tilde{p}(x, t)=p(x, s) \mathrm{e}^{s t}$ and $\tilde{u}(x, t)=u(x, s) \mathrm{e}^{s t}$. The momentum equation (3a), in the absence of forcing and in one dimension, becomes $s \bar{\rho} u=-\mathrm{d} p / \mathrm{d} x$ so the heat release term $(8 \mathrm{~b})$ becomes:

$$
\frac{\dot{q_{h}}}{\bar{p}}=n\left(\mathrm{e}^{-s \tau(x)}\right) h(x) \int_{0}^{1}-\frac{1}{s} \frac{w}{\bar{\rho}}(\breve{x}) \frac{\mathrm{d} p}{\mathrm{~d} x}(\breve{x}) \mathrm{d} \breve{x} .
$$


This is substituted into (8a), the remaining substitutions with $\mathrm{e}^{s t}$ are performed, and $w_{\bar{\rho}} \equiv$ $w / \bar{\rho}$ is defined, leading to:

$$
\frac{s^{2}}{\gamma} p-\frac{\mathrm{d}}{\mathrm{d} x}\left(\bar{v} \frac{\mathrm{d} p}{\mathrm{~d} x}\right)=-\zeta n\left(\mathrm{e}^{-s \tau(x)}\right) h(x) \int_{0}^{1} w_{\bar{\rho}}(\breve{x}) \frac{\mathrm{d} p}{\mathrm{~d} x}(\breve{x}) \mathrm{d} \breve{x}+s \epsilon \delta \dot{m}_{\bar{\rho}}-\frac{\mathrm{d}}{\mathrm{d} x} \epsilon \delta \mathbf{f}_{\bar{\rho}}+\zeta s \epsilon \delta \dot{q}_{\bar{p}}
$$

\section{F. Definition of an inner product}

An inner product is defined between two functions, $f(x)$ and $g(x)$ spanning $x \in[0,1]$ :

$$
\langle f, g\rangle \equiv \int_{0}^{1} f^{*}(x) g(x) \mathrm{d} x
$$

\section{G. Expression as an eigenvalue problem}

The terms in $\epsilon$ are now removed and will be re-introduced in $§ V I$. Equation (10) can then be written as:

$$
\begin{aligned}
\mathrm{G}(s) p & \equiv\left(\mathrm{A}(s)-s^{2} \mathrm{C}\right) p=0 \\
\text { where } \quad \mathrm{A} & \equiv \frac{\mathrm{d}}{\mathrm{d} x}\left(\bar{v} \frac{\mathrm{d}}{\mathrm{d} x}\right)-\zeta n\left(\mathrm{e}^{-s \tau}\right) h\left\langle w_{\rho}^{*}, \frac{\mathrm{d}}{\mathrm{d} x}\right\rangle \\
\text { and } \quad \mathrm{C} & \equiv 1 / \gamma .
\end{aligned}
$$

Equation (12) is satisfied for eigenvalues, $s$, with corresponding right eigenvectors, $p$.

\section{SOLVING THE DIRECT PROBLEM}

In this paper, and in the corresponding Matlab programs, the governing equations (12) are solved numerically with four different discretizations: (i) a finite difference method applied to the strong form of (12) with replacement boundary conditions, labelled FDS, (ii) a finite element method applied to the weak form of (12), labelled FEW (iii) a finite difference method applied to the weak form of (12), labelled FDW, and (iv) a summation by parts finite difference method applied to the strong form of (12) with a simultaneous approximation term for the boundary conditions, labelled SBP. A finite volume discretization has been applied to this problem by [23]. This section contains numerical details, even though they are straightforward, because they have implications for the adjoint problem. 


\section{A. Finite difference method applied to the strong form (FDS)}

A finite difference spectral method adapted from [26] is applied to the strong form of the governing equations (12). The function $p(x)$ is represented by a polynomial of order $N$. $N+1$ Gauss-Lobatto-spaced collocation points are defined at $x=x_{i}$, in the domain $x \in[0,1]$. Following the convention in [26], these are ordered backwards from $x_{N+1}$ at $x=0$ to $x_{1}$ at $x=1$. The function $p(x)$ is expressed as the sum of $N+1$ Chebyshev polynomials. The coefficients of this sum are defined uniquely by the $N+1$ values of $p\left(x_{i}\right)$. These values are held in the $(N+1)$ column vector $\mathbf{p}$. When the polynomial representing $p(x)$ is differentiated, its order reduces by 1 and therefore the polynomial representing $p^{\prime}(x)$ can be defined uniquely by the values of $p^{\prime}(x)$ at $N$ collocation points. A unique difference matrix, $\mathbf{D}$, is defined such that $\mathbf{p}^{\prime}=\mathbf{D} \mathbf{p}$. $\mathbf{D}$ has size $(N+1) \times(N+1)$ and gives $\mathbf{p}^{\prime}$ at the $N+1$ collocation points, but has rank $N$ because only $N$ values are required to define $p^{\prime}(x)$ uniquely.

The integral $\int_{0}^{1} p \mathrm{~d} x$ is given by $\mathbf{m}^{T} \mathbf{p}$, where $\mathbf{m}$ is a column vector containing the weighting associated with each collocation point. These are calculated with Clenshaw-Curtis quadrature [26, chapter 12]. A diagonal matrix $\mathbf{M}$ is defined, which contains the elements of $\mathbf{m}$ along the diagonal. For reasons described in $\S I V B$, it is convenient to pre-multiply the discretized forms of (12) by $\mathbf{M}$. This preconditioning does not affect the eigenvalues, $s$, or the right eigenvectors, $\mathbf{p}$. With this preconditioning, the discretized form of (12) is:

$$
\begin{aligned}
\mathbf{G}(s) \mathbf{p} \equiv\left(\mathbf{A}(s)-s^{2} \mathbf{C}\right) \mathbf{p} & =0 \\
\text { where } \quad \mathbf{A} & \equiv \mathbf{M D V D}-\mathbf{M} \zeta n\left(\mathbf{e}^{-s \tau}\right) \mathbf{h} \mathbf{w}_{\bar{\rho}}^{T} \mathbf{M D} \\
\text { and } \quad \mathbf{C} & \equiv \mathbf{M} / \gamma
\end{aligned}
$$

where $\mathbf{e}^{-s \tau}$ is a $(N+1) \times(N+1)$ matrix containing $\mathrm{e}^{-s \tau\left(x_{i}\right)}$ along the diagonal, $\mathbf{h}$ is a $(N+1)$ column vector containing $h\left(x_{i}\right), \mathbf{w}_{\bar{\rho}}$ is a $(N+1)$ column vector containing $w_{\bar{\rho}}\left(x_{i}\right)$, and $\mathbf{V}$ is a $(N+1) \times(N+1)$ matrix containing $\bar{v}\left(x_{i}\right)$ along the diagonal. In the Matlab programs, the collocation points, $\mathbf{x}$, differentiation matrix, $\mathbf{D}$, mass matrix, $\mathbf{M}$, density profile, $\mathbf{V}$, and heat release properties, $\mathbf{h}(x), \mathbf{w}_{\bar{\rho}}(x)$, and $\tau(x)$, are created with mat_FD.m. Matrices $\mathbf{A}$ and C in (13) are assembled within mat_AC_FDS_DA.m.

Equation (12) is a second order ODE for $p$ and therefore requires two boundary conditions. It is solved on the domain $x \in[0,1]$ with boundary conditions on $p$ at $x=0$ (upstream) and 
$x=1$ (downstream). These boundary conditions are either homogenous Dirichlet, $p=0$, homogenous Neumann, $p^{\prime}=0$, or Robin: $p^{\prime}=-k_{u} p$ at $x=0$ and $p^{\prime}=+k_{d} p$ at $x=1$. (The difference in sign arises because the surface normal at $x=0$ has the opposite sign from that at $x=1$.) The constant $k=s(\bar{\rho} / \gamma)^{1 / 2}(R-1) /(R+1)$, where $R$ is the acoustic reflection coefficient. Homogenous Dirichlet boundary conditions could be applied at $x=1$ by removing the top row and left column of $\mathbf{A}$ and $\mathbf{C}$. This has the advantage that $\mathbf{A}$ and $\mathbf{C}$ regain full rank but the practical disadvantage when coding that the matrices change size. Instead, in this paper, the replacement method is used: homogenous Dirichlet boundary conditions are applied at $x=1$ by setting the top row and left column of $\mathbf{A}$ and $\mathbf{C}$ to zero and then setting the top-left element of $\mathbf{A}$ to 1 . The same procedure is applied at $x=0$ by altering the bottom rows and right columns of $\mathbf{A}$ and $\mathbf{C}$. Homogenous Neumann boundary conditions are applied by (i) replacing the top or bottom rows of $\mathbf{A}$ by the top or bottom rows of $\mathbf{D}$ and (ii) entering zero into the top-left or bottom-right elements of $\mathbf{C}$. Robin boundary conditions $\left(p^{\prime}=k p\right)$ are applied by following steps (i) and (ii) above and then subtracting $k_{d}$ from the top-left element of $\mathbf{A}$ or $-k_{u}$ from the bottom-right element of $\mathbf{A}$. These boundary conditions are implemented in fun_bcs_strong.m. For all of these boundary conditions, the top and bottom rows of $\mathbf{A}$ can be multiplied by arbitrary (nonzero) constants, $c_{d}$ and $c_{u}$, without affecting the eigenvalue, $s$, or right eigenvector, $\mathbf{p}$. This has implications for the left eigenvector and adjoint eigenfunctions (§IV B and figure 2). The generalized matrix eigenvalue problem $\mathbf{A}(s) \mathbf{p}=s^{2} \mathbf{C p}$ is solved numerically (§III E). $\mathbf{C}$ is non-invertible due to the zeros added when the boundary conditions are applied. This creates two infinite eigenvalues, with corresponding eigenfunctions, which are discarded.

\section{B. Finite element method applied to the weak form (FEW)}

Using the inner product (11), the governing equation (12) is multiplied by a test function, $z$, which can be complex, and re-arranged via integration by parts into the weak form:

$$
\left\langle z,\left(\mathrm{~A}(s)-s^{2} \mathrm{C}\right) p\right\rangle=\left[z^{*} \bar{v} \frac{\mathrm{d} p}{\mathrm{~d} x}\right]_{0}^{1}-\left\langle\frac{\mathrm{d} z}{\mathrm{~d} x}, \bar{v} \frac{\mathrm{d} p}{\mathrm{~d} x}\right\rangle-\left\langle z, \zeta n\left(\mathrm{e}^{-s \tau}\right) h\left\langle w_{\bar{\rho}}^{*}, \frac{\mathrm{d} p}{\mathrm{~d} x}\right\rangle\right\rangle-\left\langle z, \frac{s^{2}}{\gamma} p\right\rangle=0
$$

In order to discretize (14), restrictions are now placed on the differentiability of $z$ and $p$. $N+1$ gridpoints are defined at $x=x_{1, i}$, equispaced from $x=0$ to $x=1$. The functions 
$z(x)$ and $p(x)$ are defined to be piece-wise linear (P1), with values $z_{i}$ and $p_{i}$ at the $N+1$ gridpoints at $x_{1, i}$. These are held as column vectors $\mathbf{z}_{1}, \mathbf{p}_{1}$, and $\mathbf{x}_{1}$. The derivatives $z^{\prime}(x)$ and $p^{\prime}(x)$ are therefore piece-wise constant (P0) functions, which are discontinuous at $x_{1, i}$. It is convenient to define $N$ new gridpoints, $x_{0, i}$, at the mean points between $x_{1, i}$, and to hold the values $z_{i}^{\prime}$ and $p_{i}^{\prime}$ of these $\mathrm{P} 0$ functions at the $N$ gridpoints at $x_{0, i}$. These are held as column vectors $\mathbf{z}_{0}^{\prime}, \mathbf{p}_{0}^{\prime}$, and $\mathbf{x}_{0}$. A difference matrix, $\mathbf{D}_{01}$, is defined such that $\mathbf{p}_{0}^{\prime}=\mathbf{D}_{01} \mathbf{p}_{1}$. A mean matrix, $\overline{\mathbf{M}}_{01}$, is defined such that $\mathbf{x}_{0}=\overline{\mathbf{M}}_{01} \mathbf{x}_{1}$. $\mathbf{D}_{01}$ and $\overline{\mathbf{M}}_{01}$ map P1 functions to P0 functions, so have $N$ rows and $N+1$ columns. They therefore both have rank $N$, as did the difference matrix $\mathbf{D}$ in $\S I I I A$.

The inner product $\langle f, g\rangle$ between two $\mathrm{P} 1$ functions is evaluated exactly by $\mathbf{f}^{H} \mathbf{M}_{11} \mathbf{g}$, where $\mathbf{M}_{11}$ is a $(N+1) \times(N+1)$ tri-diagonal matrix. The inner product $\langle f, g\rangle$ between two $\mathrm{P} 0$ functions is evaluated exactly by $\mathbf{f}^{H} \mathbf{M}_{00}$ g, where $\mathbf{M}_{00}$ is a $N \times N$ diagonal matrix. The inner product $\langle f, g\rangle$ between a $\mathrm{P} 0$ and a $\mathrm{P} 1$ function is evaluated exactly by $\mathbf{f}^{H} \mathbf{M}_{01}$ g where $\mathbf{M}_{01}$ is a $N \times(N+1)$ matrix. In this formulation, $\mathbf{M}_{01}=\mathbf{M}_{00} \overline{\mathbf{M}}_{01}$. Equation (14) can then be written in matrix form as

$$
\begin{aligned}
\mathbf{z}_{1}^{H} \mathbf{G}_{11}(s) \mathbf{p}_{1} & \equiv \mathbf{z}_{1}^{H}\left(\mathbf{A}_{11}-s^{2} \mathbf{C}_{11}\right) \mathbf{p}_{1}=0 \\
\text { where } \quad \mathbf{A}_{11} & \equiv-\mathbf{D}_{01}^{H} \mathbf{M}_{00} \mathbf{V}_{00} \mathbf{D}_{01}-\mathbf{M}_{11} \zeta n\left(\mathbf{e}^{-s \tau_{1}}\right) \mathbf{h}_{1} \mathbf{w}_{\bar{\rho} 0}^{T} \mathbf{M}_{00} \mathbf{D}_{01} \\
\text { and } \quad \mathbf{C}_{11} & \equiv \mathbf{M}_{11} / \gamma
\end{aligned}
$$

where $\mathbf{V}_{00}$ is a $N \times N$ matrix containing $\bar{v}\left(x_{0, i}\right)$ along the diagonal, $\mathbf{e}^{-s \tau_{1}}$ is a $(N+1) \times(N+1)$ matrix containing $\mathrm{e}^{-s \tau\left(x_{1, i}\right)}$ along the diagonal, $\mathbf{h}_{1}$ is a $N+1$ column vector containing $h\left(x_{1, i}\right)$, and $\mathbf{w}_{\bar{\rho} 0}$ is a $N$ column vector containing $w_{\bar{\rho}}\left(x_{0, i}\right)$. In the Matlab programs, the points, differentiation matrices, and mass matrices are created with mat_FE.m. Matrices $\mathbf{A}_{11}$ and $\mathrm{C}_{11}$ are assembled with mat_AC_FEW_DA.m.

The boundary conditions are applied via the boundary term in (14). For homogeneous Neumann boundary conditions, $p^{\prime}=0$, this term is zero and no further action is required. For Robin boundary conditions, this term is $z^{*} \bar{v}_{d} k_{d} p$ at $x=1$ and $-z^{*} \bar{v}_{u}\left(-k_{u}\right) p$ at $x=0$. These are applied by adding $\bar{v}_{d} k_{d}$ to the top-left element of $\mathbf{A}_{11}$ and $\bar{v}_{u} k_{u}$ to the bottom-right element of $\mathbf{A}_{11}$. Neumann and Robin boundary conditions are therefore defined without involving any arbitrary constants. Dirichlet boundary conditions are applied using the method described in $§ I I I A$, which does involve an arbitrary constant. These boundary conditions are implemented in fun_bcs_weak.m. Equation (15) must be satisfied for arbitrary $\mathbf{z}_{1}$, so 
reduces to solving the generalized matrix eigenvalue problem $\mathbf{A}_{11} \mathbf{p}_{1}=s^{2} \mathbf{C}_{11} \mathbf{p}_{1}$. The vector $\mathbf{z}_{1}$ contains $N+1$ elements, so there are $N+1$ equations with which to calculate the $N+1$ unknowns in the vector $\mathbf{p}_{1}$, and the problem is well-posed if $\mathbf{A}_{11}$ has rank $N+1$. This is satisfied unless there are Neumann boundary conditions at both ends, in which case there is also a solution with a zero eigenvalue and a uniform eigenvector, which is discarded. For each Dirichlet boundary condition, there is an infinite eigenvalue and corresponding eigenvector, which is discarded.

\section{Finite difference method applied to the weak form (FDW)}

The finite difference scheme described in $§ I I I$ A can also be applied to the weak form (14). Equation (14) can be written in matrix form as:

$$
\begin{aligned}
\mathbf{z}^{H} \mathbf{G} \mathbf{p} & \equiv \mathbf{z}^{H}\left(\mathbf{A}-s^{2} \mathbf{C}\right) \mathbf{p}=0 \\
\text { where } \quad \mathbf{A} & \equiv-\mathbf{D}^{H} \mathbf{M V D}-\mathbf{M} \zeta n\left(\mathbf{e}^{-s \tau}\right) \mathbf{h} \mathbf{w}_{\bar{\rho}}^{T} \mathbf{M D} \\
\text { and } \quad \mathbf{C} & \equiv \mathbf{M} / \gamma
\end{aligned}
$$

where the matrices are those from the Finite Difference method $\S I I I A$ and the boundary conditions are those from the Finite Element method $\S$ III B. Matrices A and $\mathbf{C}$ are assembled with mat_AC_FDW_DA.m.

\section{Summation by Parts Finite Difference method applied to the strong form (SBP)}

In section IV B it will be shown that the Finite Difference method applied to the strong form equations with replacement boundary conditions (FDS), described in $\S$ III A, produces oscillations in the adjoint eigenvectors near the boundaries. This is because the FDS method is not dual consistent. One way to achieve a dual consistent method is to approximate

the spatial derivatives with central finite difference operators that satisfy a summation by parts (SBP) formula, and then implement the boundary conditions with the Simultaneous Approximation Term (SAT) method [27-30].

Equation (12) requires a first derivative matrix $\mathbf{D}_{1}$ representing $\mathrm{d} / \mathrm{d} x$ and a second derivative matrix with a non-homogenous coefficient, $\mathbf{D}_{2}^{(\bar{v})}$ representing $\mathrm{d} / \mathrm{d} x(\bar{v}(x) \mathrm{d} / \mathrm{d} x)$. This sec- 
ond derivative matrix could be formed by applying the first derivative twice [31, pp506-507] but this leads to a wide stencil and, unless implemented carefully, to spurious oscillations. Alternatively, the second derivative matrix can be formed with a narrow stencil in which $\bar{v}\left(x_{i}\right)$ is embedded within the stencil [32, Appendix B]. This narrow stencil avoids these spurious oscillations. Both $\mathbf{D}_{1}$ and $\mathbf{D}_{2}^{(\bar{v})}$ must satisfy the SBP formula: $\mathbf{D}_{1}$ must be expressable as $\mathbf{D}_{1}=\mathbf{M}^{-1} \mathbf{Q}$, where $\mathbf{Q}+\mathbf{Q}^{T}=\operatorname{diag}(+1,0, \ldots, 0,-1)$ when $\mathbf{x}$ is ordered from +1 to 0; similarly, $\mathbf{D}_{2}^{(\bar{v})}$ must be expressable as $\mathbf{D}_{2}^{(\bar{v})}=\mathbf{M}^{-1}\left(-\mathbf{Y}^{(b)}+\tilde{\mathbf{V}} \mathbf{S}\right)$, where $\mathbf{M}$ is the mass matrix $\operatorname{diag}(0.5,1, \ldots, 1,0.5) \times \mathrm{d} x, \mathbf{Y}^{(\bar{v})}$ is akin to a central difference second order derivative matrix, weighted by $\bar{v}\left(x_{i}\right)$, as described next, $\tilde{\mathbf{V}}=\operatorname{diag}\left(-v_{d}, 0, \ldots, 0, v_{u}\right)$, and $\mathbf{S}$ approximates the first derivative operator at the boundaries.

In this paper, the second order accurate central difference scheme of Ref. [32, Appendix B] is applied, which satisfies a SBP formula. The matrix $\mathbf{Y}^{(\bar{v})}$, which is denoted $M^{(b)}$ in [32], can be written as $\mathbf{D}_{01}^{H} \mathbf{M}_{00} \overline{\mathbf{V}}_{00} \mathbf{D}_{01}$, where $\mathbf{D}_{01}$ and $\mathbf{M}_{00}$ are identical to the matrices used in the Finite Element method (FEW) in section III B. The matrix $\overline{\mathbf{V}}_{00}$ is a $(N \times N)$ diagonal matrix containing $\left(\bar{v}\left(x_{i}\right)+\bar{v}\left(x_{i+1}\right)\right) / 2$ along the diagonal - i.e. the mean of the base density evaluated at adjacent gridpoints. To an excellent approximation, $\overline{\mathbf{V}}_{00}$ can be replaced by $\mathbf{V}_{00}$, which is a $(N \times N)$ diagonal matrix containing $\bar{v}\left(\left(x_{i}+x_{i+1}\right) / 2\right)$ along the diagonal - i.e. the base density evaluated at the mean of adjacent gridpoints. With this approximation, the SBP form of the strong form finite difference equations (12) is:

$$
\begin{aligned}
\mathbf{G}(s) \mathbf{p} & \equiv\left(\mathbf{A}-s^{2} \mathbf{C}\right) \mathbf{p}=0 \\
\text { where } \quad \mathbf{A} & \equiv-\mathbf{D}_{01}^{H} \mathbf{M}_{00} \mathbf{V}_{00} \mathbf{D}_{01}+\tilde{\mathbf{V}} \mathbf{S}-\mathbf{M} \zeta n\left(\mathbf{e}^{-s \tau}\right) \mathbf{h} \mathbf{w}_{\bar{\rho}}^{T} \mathbf{M D}_{1} \\
\text { and } \quad \mathbf{C} & \equiv \mathbf{M} / \gamma
\end{aligned}
$$

where $\mathbf{e}^{-s \tau_{1}}$ is a $(N+1) \times(N+1)$ matrix containing $\mathrm{e}^{-s \tau\left(x_{1, i}\right)}$ along the diagonal, $\mathbf{h}$ is a $N+1$ column vector containing $h\left(x_{1, i}\right)$, and $\mathbf{w}_{\bar{\rho}}$ is a $N+1$ column vector containing $w_{\bar{\rho}}\left(x_{1, i}\right)$. In the Matlab programs, the points, differentiation matrices, and mass matrices are created with mat_SBP.m. Matrices $\mathbf{A}$ and $\mathbf{C}$ are assembled with mat_AC_SBP_DA.m.

Robin and Neumann boundary conditions are applied by adding a Simultaneous Approximation Term (SAT) to the top and bottom rows of $\mathbf{A}$ :

$$
\mathbf{A} \leftarrow \mathbf{A}-\operatorname{diag}(0, \ldots, 0,1)\left(-k_{u} \bar{v}_{u} \mathbf{I}+\tilde{\mathbf{V}} \mathbf{S}\right)-\operatorname{diag}(1,0, \ldots, 0)\left(-k_{d} \bar{v}_{d} \mathbf{I}+\tilde{\mathbf{V}} \mathbf{S}\right)
$$

This is adapted from [31, Eq. (A.3)] and [32, Eq. (3.7)]. Weak Dirichlet boundary condi- 
tions can be applied with equation (3.8) of [32] or equivalently by giving $k_{u}$ and $k_{d}$ large magnitudes.

\section{E. Iteration procedures}

The nonlinear matrix eigenvalue problems $(13-17)$ are solved iteratively using two procedures, both of which are applied within fun_Helm.m. The first is a Newton method applied to the nonlinear eigenvalue problem $|\mathbf{G}(s)|=0$, using Jacobi's formula for the derivative of a determinant of a matrix:

$$
s_{j+1}=s_{j}-|\mathbf{G}| /(\mathrm{d}|\mathbf{G}| / \mathrm{d} s)_{j}=s_{j}-1 /\left(\operatorname{trace}\left(\mathbf{G}^{-1} \mathrm{~d} \mathbf{G} / \mathrm{d} s\right)\right)_{j} .
$$

This is rendered more stable by performing a QR decomposition on G. No relaxation is used. Once the eigenvalue $s$ has been found to sufficient tolerance, the eigenvector is the null space of $\mathbf{G}(s)$.

The second is the 'active iterative' method described in section IV.B of [1]. From a starting point, labelled $s_{0}$, subsequent solutions, labelled $s_{j}$, are obtained by solving the generalized linear eigenvalue problem $\mathbf{A}\left(s_{j-1}\right) \mathbf{p}=s_{j}^{2} \mathbf{C p}$ in which the frequency-dependent terms in $\mathbf{A}$ have been evaluated at $s_{j-1}$. This process is repeated $J$ times. The eigenvector is calculated alongside the eigenvalue using fun_eig_nearest.m. In both cases there are several possible solutions, and the converged solution depends on the choice of $s_{0}$. In this paper chamber modes [10] are being modelled, so it is appropriate to start from one of the natural acoustic modes of the chamber. This mode is specified by the user in fun_param_dim.m.

\section{F. Results}

The methods in this paper are demonstrated on (i) a model of the electrically-heated Rijke tube in [33], and (ii) a simplified model of a rocket engine. For the Rijke tube, $n$ is small and the peaks of $w_{\bar{\rho}}(x)$ and $h(x)$ are close, which causes the system to be nearly self-adjoint. For the rocket engine, $n$ is large and the peaks of $w_{\bar{\rho}}(x)$ and $h(x)$ are widely separated, which causes the system to be strongly non-self-adjoint. It is instructive to compare the two. The dimensional quantities, reference quantities, non-dimensional quantities and distributions

of $\bar{\rho}(x), h(x)$, and $w_{\bar{\rho}}(x)$ are listed in Table VI. The dimensional quantities are held in 

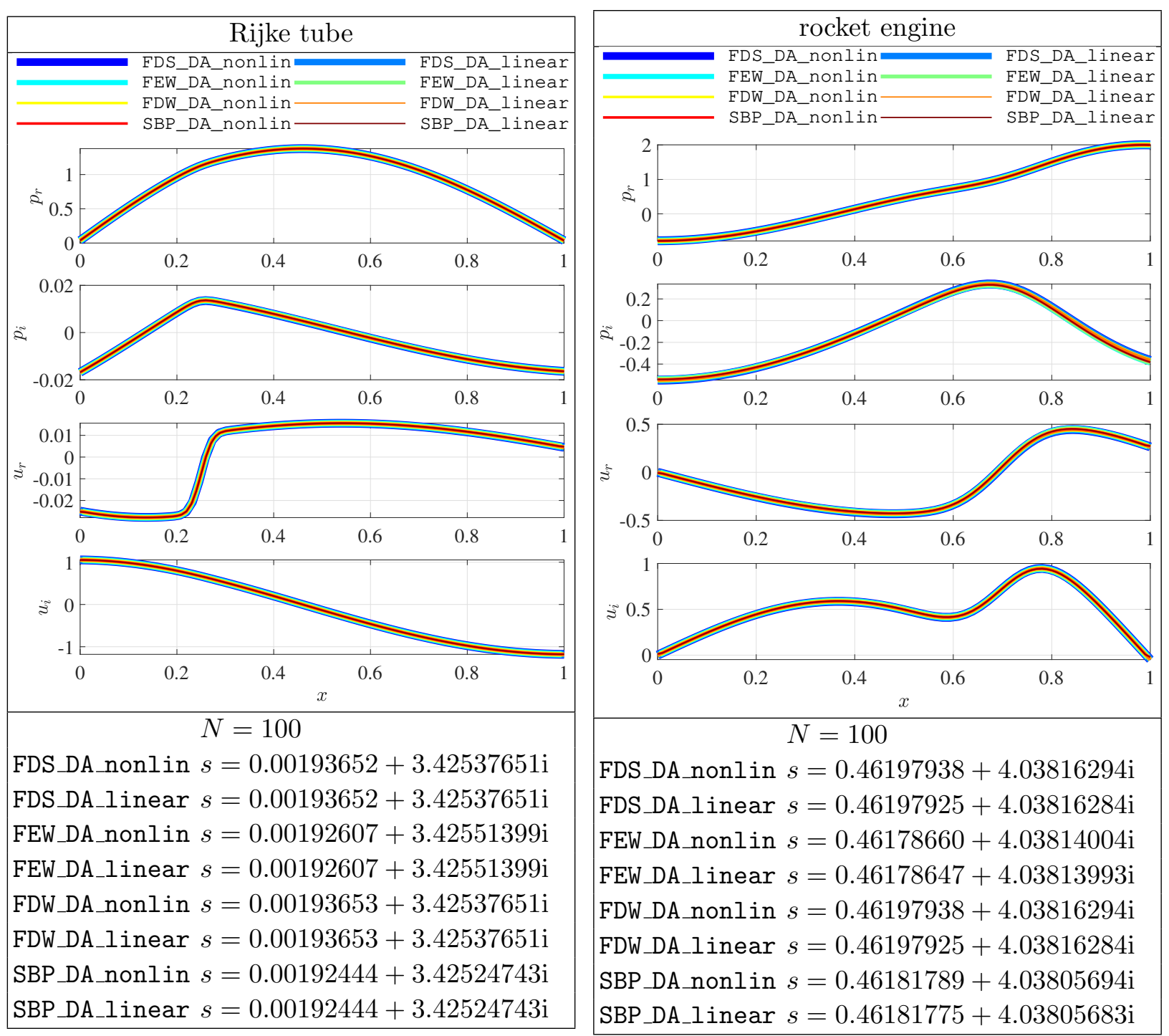

FIG. 1. (Color online) Direct pressure, $p$, and velocity, $u$, eigenfunctions calculated with four different spatial discretizations (FDS, FEW, FDW, and SBP ) and two iteration procedures (nonlin and linear). All eight lines lie on top of each other and all eigenvalues are the same to the requested tolerances. This figure is created with Fig_001.m. The agreement between the methods can be improved by increasing $N$.

fun_param_dim.m and converted to reference quantities and non-dimensional quantities in fun_param_nondim.m. The distributions are calculated in fun_rh.m, fun_h.m, and fun_wr.m.

The first eigenmode of each test case is shown in figure 1, calculated with finite difference of the strong form (FDS), finite element (FEW), finite difference of the weak form (FDW), and summation by parts finite difference of the strong form (SBP) using the nonlinear (nonlin) and linear (linear) iteration procedures. For presentation, the eigenfunctions have been 
normalized such that $\int p^{2} \mathrm{~d} x=1$. In each plot there are eight lines, which all lie on top of each other, showing that the eigenfunctions are identical to the eye. Studying the eigenvalues reveals that the two iteration procedures (linear and nonlin) converge to the same eigenvalues for each spatial discretization. The eigenvalues depend slightly on whether the Robin boundary conditions are enforced through replacement (FDS) or not (FEW, FDW, SBP). Enforcing them through replacement is more accurate, with a boundary error of order $10^{-12}$ for FDS, as compared to a boundary error of order $10^{-3}$ for FDS, FEW, and FDW. This boundary error is displayed by toggling show_boundary_error in fun_Helm.m.

\section{SOLVING THE ADJOINT PROBLEM}

\section{A. Definition of the adjoint eigenfunction and the left eigenvector}

Equation (12) is the direct continuous eigenvalue problem in the strong form. Using the inner product $(11)$, this can be pre-multiplied by a function $p^{\dagger}(x)$ :

$$
\left\langle p^{\dagger}, \mathrm{G}(s) p\right\rangle \equiv\left\langle p^{\dagger},\left(\mathrm{A}(s)-s^{2} \mathrm{C}\right) p\right\rangle=0
$$

For a given eigenvalue, $s$, the corresponding direct eigenfunction, $p(x)$, satisfies (20) for arbitrary $p^{\dagger}(x)$, and the corresponding adjoint eigenfunction, $p^{\dagger}(x)$, satisfies (20) for arbitrary $p(x)$. This is the definition of the adjoint eigenfunction $p^{\dagger}(x)$.

There is an equivalent definition for the discretized problem. The corresponding generalized matrix eigenvalue problem (13) is premultiplied by a column vector $\mathbf{z}^{H}$ :

$$
\mathbf{z}^{H} \mathbf{G}(s) \mathbf{p} \equiv \mathbf{z}^{H}\left(\mathbf{A}(s)-s^{2} \mathbf{C}\right) \mathbf{p}=0
$$

For a given eigenvalue, $s$, the corresponding right eigenvector $\mathbf{p}$, satisfies (21) for arbitrary $\mathbf{z}$, and the corresponding left eigenvector, $\mathbf{z}$, satisfies (21) for arbitrary $\mathbf{p}$. This is the definition of the left eigenvector $\mathbf{z}$. By taking the Hermitian of (21), it is easily shown that the left eigenvectors of the direct problem $\left(\mathbf{A}-s^{2} \mathbf{C}\right)$ are the right eigenvectors of the adjoint

problem $\left(\mathbf{A}^{H}-s^{* 2} \mathbf{C}^{H}\right)$. This identity can be used if a matrix eigenvalue solver does not compute the left eigenvectors automatically. 


\section{B. Discrete adjoint of the strong form (FDS_DA, SBP_DA)}

The discretized form of $(20)$ is:

$$
\mathbf{p}^{\dagger^{H}} \mathbf{M G p} \equiv \mathbf{p}^{\dagger^{H}} \mathbf{M}\left(\mathbf{A}-s^{2} \mathbf{C}\right) \mathbf{p}=0
$$

where $\mathbf{p}^{\dagger}$ contains the values of the adjoint eigenfunction, $p^{\dagger}\left(x_{i}\right)$, at the collocation points and $\mathbf{M}$ is the mass matrix. In $\S$ III $\mathrm{A}$ and $\S I I I D$, the discretized forms of $\mathrm{A}$ and $\mathrm{C}$ in $(12)$ were pre-multiplied by $\mathbf{M}$ to create $\mathbf{A}$ and $\mathbf{C}$ in (13) and (17). This ensures that the left eigenvectors of the discretized problem, $\mathbf{z}$ in (21), are the values of the adjoint eigenfunction, $p^{\dagger}\left(x_{i}\right)$ in $(20)$, at the collocation points.

The advantage of finding the adjoint eigenfunction through this approach, which is known as the discrete adjoint approach, is that the direct and adjoint problems use the same matrices $\mathbf{A}$ and $\mathbf{C}$ and therefore contain the same discretization and truncation errors, and have the same eigenvalues to machine precision. Another advantage is that the boundary conditions are already embedded within $\mathbf{A}$ and $\mathbf{C}$ and do not need to be evaluated separately. The disadvantage is that, when Neumann or Robin boundary conditions are applied by replacing rows in $\mathbf{A}$ and $\mathbf{C}$, as done for FDS in $§ I I I A$, (i) the left eigenvector takes an arbitrary value at the wall and (ii) the left eigenvector oscillates around the boundary, for as many gridpoints as there are in the stencil in the difference matrix $\mathbf{D}$. This disadvantage can be avoided by ensuring that the $\mathbf{A}$ matrix satisfies a summation by parts formula and that the boundary conditions are applied with the Simultaneous Approximation Term, as described in $\S I I I D$ for SBP.

The first disadvantage of the FDS_DA method arises because Dirichlet, Neumann, and Robin boundary conditions are imposed by replacing the bottom row of $\mathbf{A}$ with the boundary condition on $p$ and setting the bottom-right element of $\mathbf{C}$ to zero. This causes $\mathbf{C}$ to become non-invertible, which results in a right eigenvector with an infinite eigenvalue. This eigenmode is discarded. For all types of boundary condition, the bottom row of $\mathbf{A}$ can be multiplied by an arbitrary constant, $c_{u}$, without affecting the eigenvalues, $s$, or the right eigenvectors, $\mathbf{p}$ (i.e. the thick blue lines in figure 1 do not depend on $c_{u}$.) The arbitrary constant does, however, affect the corresponding left eigenvectors, z. For Dirichlet boundary conditions, the arbitrary constant only affects one left eigenvector: that which is discarded because it has an infinite eigenvalue. All the physical left eigenvectors are unaffected. For

Neumann and Robin boundary conditions, however, every left eigenvector depends on the 
arbitrary constant. This can be seen in figure 2, which shows the adjoint eigenfunctions calculated with this method (the dark blue line) and the methods described in $\S I V \mathrm{C}$ and $\S I V D$ (the remaining seven colored lines). The supplementary file Sup_001.m plots the direct and adjoint eigenfunctions at different values of $c_{u}$. This shows that the direct eigenfunction is unaffected by $c_{u}$ while the adjoint eigenfunction at the boundary is strongly affected by $c_{u}$. This problem can be anticipated by examining (20) and recalling that A contains $\mathrm{d}^{2} / \mathrm{d} x^{2}$. Therefore, if one attempts to find solutions for $p^{\dagger}$ and $p$ in the same function space, the inner product is formed between two functions in different function spaces - i.e. with different numbers of degrees of freedom. In this case, this means that $p$ can be found unambiguously, but that $p^{\dagger}$ can be found only up to two arbitrary constants, which in this case are $c_{u}$ and $c_{d}$. This disadvantage is not serious because it does not affect the base state or feedback sensitivities $(\S \mathrm{VC})$.

The second disadvantage of the FDS_DA method arises because, in $\mathbf{p}^{\dagger^{H}} \mathbf{M G}$ in $(22)$, the left eigenvector acts on the columns (rather than the rows) of $\mathbf{G}$. The matrix $\mathbf{G}$ contains $\mathbf{D}$, which is a difference matrix in which row $i$ performs a difference scheme to evaluate $(\mathrm{d} / \mathrm{d} x)$ at point $i$, which has a clear physical meaning. When $\mathbf{p}^{\dagger^{H}}$ acts on column $j$ of $\mathbf{D}$, however, the result is the sum of the contribution of point $j$ to every difference scheme. This has no physical meaning unless a central difference scheme is used consistently at every point, in which case $\mathbf{D}^{H}=\mathbf{D}$ and $\mathbf{p}^{\dagger H}$ acting on column $j$ of $\mathbf{D}$ gives $\mathrm{d} p^{\dagger} / \mathrm{d} x$ at point $j$. In general, however, there is no need for the difference schemes in each row to be consistent with each other - i.e. there is no need for $\mathbf{D}$ to be Hermitian. (For example, a central difference scheme must shift to a downwind scheme as its stencil impinges on the upstream boundary.) If the difference schemes in each row are not consistent with each other then the value of $\mathbf{p}^{\dagger^{H}}$ acting on column $j$ of $\mathbf{D}$ contains contributions from different difference schemes. In practice this leads to oscillations appearing in $\mathbf{p}^{\dagger}$ if $\mathbf{p}^{\dagger}$ is not zero at the boundary (i.e. when non-Dirichlet boundary conditions are applied). These oscillations occur around the boundaries for as many gridpoints as are in the stencil of the difference scheme. For the Chebyshev difference scheme used in this paper, $\mathbf{D}$ is a full matrix with a different difference scheme in each row. These oscillations therefore extend into the whole domain, as can be seen for FDS_DA in Fig. 2. Their amplitude, but not their shape, depends on the value at the boundary and therefore on $c_{u}$ and $c_{d}$. 


\section{Discrete adjoint of the weak form (FEW_DA, FDW_DA)}

The continuous equations (12) can be integrated by parts once to give the weak form (14). When discretized, this becomes $(15,16)$. The left eigenvectors of $(15 \mathrm{a}, 16 \mathrm{a})$, $\mathbf{z}$, are the adjoint eigenfunctions, $p^{\dagger}\left(x_{i}\right)$ evaluated at the collocation points, $x_{i}$. These are arranged in the column vector $\mathbf{p}^{\dagger}$. This approach has all the advantages of the discrete adjoint in the strong form but does not have its disadvantages. Firstly this is because $\mathbf{p}^{\dagger}$ acts on $\mathbf{D}^{H}$, rather than D. Secondly this is because $s, \mathbf{p}$, and $\mathbf{p}^{\dagger}$ are defined uniquely, without involving any arbitrary constants. (An arbitrary constant is involved in imposing Dirichlet boundary conditions but this only affects the discarded eigenmode). Alternatively, this outcome can be anticipated by examining (14) and noting that each inner product is between functions that exist in the same function space. This means that $p^{\dagger}$ and $p$ can be found unambiguously, without involving arbitrary constants.

\section{Continuous adjoint in the strong form}

Having defined the adjoint eigenfunction in (20), the test function $z$ in (14) is now replaced with the adjoint eigenfunction $p^{\dagger}$ and equation (14) is integrated by parts a second time to give the strong form of the continuous adjoint equations:

$$
\begin{aligned}
\left\langle p^{\dagger},\left(\mathrm{A}-s^{2} \mathrm{C}\right) p\right\rangle & =\left[p^{\dagger^{*}} \bar{v} \frac{\mathrm{d} p}{\mathrm{~d} x}-\bar{v} \frac{\mathrm{d} p^{\dagger^{*}}}{\mathrm{~d} x} p-w_{\bar{\rho}}\left\langle p^{\dagger}, \zeta n\left(\mathrm{e}^{-s \tau}\right) h\right\rangle p\right]_{0}^{1}+\left\langle\left(\mathrm{A}^{\dagger}-s^{* 2} \mathrm{C}^{\dagger}\right) p^{\dagger}, p\right\rangle=0 \\
\text { where } \mathrm{A}^{\dagger} p^{\dagger} & \equiv \frac{\mathrm{d}}{\mathrm{d} x}\left(\bar{v}^{*} \frac{\mathrm{d} p^{\dagger}}{\mathrm{d} x}\right)+\left(\frac{\mathrm{d} w_{\bar{\rho}}^{*}}{\mathrm{~d} x}\right)\left\langle\zeta n\left(\mathrm{e}^{-s \tau}\right) h, p^{\dagger}\right\rangle \\
\text { and } \mathrm{C}^{\dagger} & \equiv 1 / \gamma^{*} .
\end{aligned}
$$

Equation (23) must be satisfied for arbitrary $p$. The inner product term in (23a) provides the continuous adjoint equations $\left(\mathrm{A}^{\dagger}-s^{* 2} \mathrm{C}^{\dagger}\right) p^{\dagger}=0$. The eigenvalue of the adjoint problem is the complex conjugate of that of the direct problem. The boundary term in (23a) provides the boundary conditions for the adjoint eigenfunction, $p^{\dagger}$, in terms of those for the direct eigenfunction $p$. For homogenous Dirichlet boundary conditions on $p$, the boundary term requires homogenous Dirichlet boundary conditions on $p^{\dagger}$, by inspection. For Robin 
boundary conditions $\mathrm{d} p / \mathrm{d} x=k p$, each boundary must satisfy:

$$
p^{\dagger^{*}} \bar{v} k-\bar{v} \frac{\mathrm{d} p^{\dagger^{*}}}{\mathrm{~d} x}-w_{\bar{\rho}}\left\langle p^{\dagger}, \zeta n\left(\mathrm{e}^{-s \tau}\right) h\right\rangle=0
$$

If the measurement function, $w_{\bar{\rho}}$, smoothly tends to zero at the boundary then this simply requires $\mathrm{d} p^{\dagger} / \mathrm{d} x=k^{*} p^{\dagger}$, which are Robin boundary conditions on $p^{\dagger}$. However, if $w_{\bar{\rho}}$ does not smoothly tend to zero at the boundary then the boundary condition on $p^{\dagger}$ requires information about $p^{\dagger}$ in the entire domain, through the inner product $\left\langle p^{\dagger}, \zeta n\left(\mathrm{e}^{-s \tau}\right) h\right\rangle$. Solving this is difficult and it is therefore wise to move the boundary to ensure that $w_{\bar{\rho}}$ smoothly tends to zero at the boundary. (This effect can be seen particularly clearly by comparing the CA and DA eigenvalues for the model rocket when the measurement location $X_{w}$ is moved close to the inlet.)

The continuous approach has the advantage that the adjoint eigenfunction, $p^{\dagger}$, can unambiguously be calculated in the same function space as the direct eigenfunction, $p$, without the use of arbitrary constants. It has two slight disadvantages. First, the boundary conditions have to be evaluated and implemented separately, which can be challenging and error-prone. Second, the direct and adjoint problems can have different discretizations, different truncation errors, and different solution algorithms. This means that the direct and adjoint eigenvalues are not identical to machine precision. This precludes the use of stringent convergence tests to debug the continuous adjoint code $(\S \mathrm{VF})$. It may be convenient to write a discrete adjoint code in order to debug a continuous adjoint code, which was the approach taken in [34].

The continuous approach also provides a physical interpretation of the adjoint variable. The heat release term of the direct equation $(12 \mathrm{~b})$ can be integrated by parts once to give

$$
\mathrm{A} p=\frac{\mathrm{d}}{\mathrm{d} x}\left(\bar{v} \frac{\mathrm{d} p}{\mathrm{~d} x}\right)+\zeta n\left(\mathrm{e}^{-s \tau}\right) h\left\langle\frac{\mathrm{d} w_{\bar{\rho}}^{*}}{\mathrm{~d} x}, p\right\rangle
$$

if, as is likely, $h=0$ at the boundaries. The acoustic terms (the first terms) in (23b) and (25) are the same. This is expected because acoustic equations are self-adjoint in the absence of damping and forcing. The heat release terms (the second terms) in (23b) and (25) are mirror images of each other. In the direct equation (25), the amplitude of the forcing is determined by the integral of the pressure and the gradient of the measurement function, $w_{\bar{\rho}}^{\prime}$, while its position is determined by the heat release function, $\left(\mathrm{e}^{-s \tau}\right) h(x)$. In brief, this term is influenced by the measurement region and influences the heat release 
region. In the adjoint equation (23b), the amplitude of the forcing is determined by the integral of the adjoint pressure and heat release function, while its position is determined by the gradient of the measurement function. In brief, this term is influenced by the heat release region and influences the measurement region. This shows that the system becomes more non-self-adjoint the more that $\left(\mathrm{e}^{-s \tau}\right) h$ differs from $w_{\bar{\rho}}^{\prime}$. This difference is caused by the spatial separation of the peaks of $h(x)$ and $w(x)$, and the time delay, $\tau(x)$. This has physical implications (§VIII). Note also that if the measurement region is a Dirac delta: $w(x)=\delta\left(x-x_{r e f}\right)$, as is often the case in practical implementations, then the heat release term in the above adjoint equation contains the derivative of a Dirac delta. It is then advisable to integrate this term by parts so that this becomes the evaluation of $\mathrm{d} p / \mathrm{d} x$ at $x_{w}$. In this paper $x_{w}$ is expanded to a measurement region in order to avoid this.

\section{E. Implementation of the continuous adjoint equations $*_{-} \mathrm{CA}$}

A column vector $\mathbf{w}_{\bar{\rho}}^{\prime}$ is defined to contain $\mathrm{d} w_{\bar{\rho}} / \mathrm{d} x$ at the collocation points. The discretized forms of $(23)$ are:

$$
\mathbf{G}^{\dagger}(s) \mathbf{p}^{\dagger} \equiv\left(\mathbf{A}^{\dagger}(s)-s^{* 2} \mathbf{C}^{\dagger}\right) \mathbf{p}^{\dagger}=0
$$

where, for Finite Difference of the strong form (mat_AC_FDS_CA.m),

$$
\begin{aligned}
& \mathbf{A}^{\dagger} \equiv \mathbf{M D V D}+\mathbf{M} \zeta n \mathbf{w}_{\bar{\rho}}^{\prime} \mathbf{h}^{H}\left(\mathbf{e}^{-s^{*} \tau^{*}}\right) \mathbf{M} \\
& \mathbf{C}^{\dagger} \equiv \mathbf{M} / \gamma
\end{aligned}
$$

for Finite Element of the weak form (mat_AC_FEW_CA.m),

$$
\begin{aligned}
& \mathbf{A}_{11}^{\dagger} \equiv-\mathbf{D}_{01}^{H} \mathbf{M}_{00} \mathbf{V}_{00} \mathbf{D}_{01}+\mathbf{M}_{11} \zeta n \mathbf{w}_{\bar{\rho} \mathbf{1}}^{\prime} \mathbf{h}_{1}^{H}\left(\mathbf{e}^{-s^{*} \tau_{1}^{*}}\right) \mathbf{M}_{11} \\
& \mathbf{C}_{11}^{\dagger} \equiv \mathbf{M}_{11} / \gamma
\end{aligned}
$$

for Finite Difference of the weak form (mat_AC_FDW_CA.m),

$$
\begin{aligned}
& \mathbf{A}^{\dagger} \equiv-\mathbf{D}^{H} \mathbf{M V D}+\mathbf{M} \zeta n \mathbf{w}_{\bar{\rho}}^{\prime} \mathbf{h}^{H}\left(\mathbf{e}^{-s^{*} \tau^{*}}\right) \mathbf{M} \\
& \mathbf{C}^{\dagger} \equiv \mathbf{M} / \gamma
\end{aligned}
$$


and for Finite Difference of the strong form obeying a Summation by Parts formula and with the Simultaneous Approximation Term (mat_AC_SBP_CA.m),

$$
\begin{aligned}
& \mathbf{A}^{\dagger} \equiv-\mathbf{D}_{01}^{H} \mathbf{M}_{00} \mathbf{V}_{00} \mathbf{D}_{01}+\tilde{\mathbf{V}} \mathbf{S}+\mathbf{M} \zeta n \mathbf{w}_{\bar{\rho}}^{\prime} \mathbf{h}^{H}\left(\mathbf{e}^{-s^{*} \tau^{*}}\right) \mathbf{M} \\
& \mathbf{C}^{\dagger} \equiv \mathbf{M} / \gamma .
\end{aligned}
$$

The boundary conditions are applied in the same way as for the direct equations, but with the complex conjugates of $k_{u}$ and $k_{d}$ for Robin boundary conditions.

\section{F. Iteration Procedures}

For the discretized direct systems (13-17), the left eigenvector $\mathbf{z}$ in (21) contains the values of the adjoint eigenfunction at the gridpoints, $p^{\dagger}\left(x_{i}\right)$ in $(20)$, as explained in $\S$ IV B and $§ I V C$. For the Newton method, this left eigenvector is found by calculating the null space of $\mathbf{G}^{H}$. For the active iteration method, this left eigenvector is calculated alongside the right eigenvector using fun_eig_nearest.

For the continuous adjoint equations (27-30), solutions are found in the same ways as for the direct problem: (i) the Newton method applied to the nonlinear eigenvalue problem $\left|\mathbf{G}^{\dagger}(s)\right|=0$; (ii) the active iteration method applied to the sequence of linear eigenvalue

problems $\mathbf{A}^{\dagger}\left(s_{j-1}\right) \mathbf{p}^{\dagger}=s_{j}^{* 2} \mathbf{C}^{\dagger} \mathbf{p}^{\dagger}$. In each case, the values of the adjoint eigenfunction at the gridpoints, $p^{\dagger}\left(x_{i}\right)$ in $(20)$, are the components of the right eigenvector $\mathbf{p}^{\dagger}$.

\section{G. Results}

Figure 2 shows the adjoint eigenfunctions calculated with the discrete (DA) and continuous (CA) adjoint approaches for finite difference in the strong form (FDS), finite element (FEW), finite difference in the weak form (FDW), and summation by parts finite difference (SBP). For presentation, they have been normalized such that $\int_{0}^{1} p^{\dagger^{2}} \mathrm{~d} x=1$. From a computational point of view, the main point is that all the methods give the same results but that the discrete adjoint of the finite difference of the strong form (FDS_DA) gives arbitrary values at the boundary and oscillations around the boundary, as described in §IV B. From a physical point of view, figure 2 shows that the adjoint pressure eigenfunction, $p^{\dagger}$, changes abruptly at the measurement region, while the direct pressure and velocity eigenfunctions, 
$p$ and $u$ in figure 1, change at the heat release region. These features can be anticipated by comparing (23b) with (25) and show clearly that the problem is not self-adjoint. The adjoint eigenfunctions are used to calculate the base state sensitivities $(\S \mathrm{V})$, the feedback sensitivities (§VI), and the receptivities (§VII).

\section{BASE STATE SENSITIVITY ANALYSIS}

In this section, the theory and numerics of the base state sensitivity analysis is described for the continuous framework ( $(\mathrm{VA})$, and the discrete framework ( $\S \mathrm{VB})$, in the FDS, FEW, FDW, and SBP formulations. The physical interpretation of the base state sensitivities is in $\S$ VIII C.

\section{A. Base state sensitivities in the continuous framework}

The direct equation (12) is an eigenvalue problem $\mathrm{G}(s) p=0$, possibly with boundary conditions that depend on $s$. If the operator, $\mathrm{G}(s)$, is perturbed to $\mathrm{G}+\epsilon \delta \mathrm{G}$ then each eigenvalue shifts to $s+\epsilon \delta s$ and its corresponding direct eigenfunction to $p+\epsilon \delta p$. The governing equations must still be satisfied, so:

$$
\left(\mathrm{G}(s)+\epsilon \delta s \frac{\mathrm{dG}}{\mathrm{d} s}+\epsilon \delta \mathrm{G}\right)(p+\epsilon \delta p)+\mathcal{O}\left(\epsilon^{2}\right)=0 \quad .
$$

This is correct for non-degenerate eigenvalues. The sensitivity of degenerate eigenvalues requires a different approach [35]. Considering terms at order $\epsilon$ gives:

$$
\mathrm{G} \delta p+\delta s \frac{\mathrm{dG}}{\mathrm{d} s} p+(\delta \mathrm{G}) p=0
$$

Using the inner product (11), this expression is pre-multiplied by the corresponding adjoint eigenfunction, which is defined such that $\left\langle p^{\dagger}, \mathrm{G} f\right\rangle=0$ for any $f$, and re-arranged to:

$$
\begin{aligned}
\delta s & =\frac{\left\langle p^{\dagger},(\delta \mathrm{G}) p\right\rangle}{\alpha} \\
\text { where } \quad \alpha & \equiv\left\langle p^{\dagger},-\frac{\mathrm{dG}}{\mathrm{d} s} p\right\rangle=\left\langle p^{\dagger},-\left(\left.\frac{\partial \mathrm{G}}{\partial s}\right|_{k}+\left.\frac{\mathrm{d} k}{\mathrm{~d} s} \frac{\partial \mathrm{G}}{\partial k}\right|_{s}\right) p\right\rangle .
\end{aligned}
$$

The final term in (33b) accounts explicitly for frequency-dependent Robin boundary conditions. From (12), $\partial \mathrm{G} /\left.\partial s\right|_{k}=\zeta n\left(\tau \mathrm{e}^{-s \tau}\right) h\left\langle w_{\rho}^{*}, \mathrm{~d} / \mathrm{d} x\right\rangle-2 s \mathrm{C}$. In order to make $k$ appear explicitly in $(33 \mathrm{~b})$, the equation is integrated by parts to obtain $(\mathrm{d} k / \mathrm{d} s) \times(\partial / \partial k)$ of $(14)$. 
Then $\mathrm{d} p / \mathrm{d} x$ in the boundary term is replaced with $k p$. Then this is differentiated with respect to $k$. If $w_{\bar{\rho}}^{*}=0$ at the boundaries, which is good practice because of $(24)$, the final expression for $\alpha$ is:

$$
\alpha=-\left\langle p^{\dagger}, \zeta n\left(\tau \mathrm{e}^{-s \tau}\right) h\left\langle w_{\rho}^{*}, \frac{\mathrm{d} p}{\mathrm{~d} x}\right\rangle-2 s \mathrm{C}\right\rangle-p^{\dagger}{ }_{u} \bar{v}_{u} \frac{\mathrm{d} k_{u}}{\mathrm{~d} s} p_{u}-p^{\dagger}{ }_{d} \bar{v}_{d} \frac{\mathrm{d} k_{d}}{\mathrm{~d} s} p_{d}
$$

where the final two terms are required only for frequency-dependent Robin boundary conditions.

The numerator of (33a) is considered next. The operator $\mathrm{G}$ depends on the base state variables $\bar{v}(x), n, \tau(x), h(x), w_{\bar{\rho}}(x)$, described generically as $b(x)$, and the Robin boundary conditions $k_{u}, k_{d}$, described generically as $k$. If these base state variables are each perturbed by $\epsilon$ then the numerator of (33) is found by setting $z=p^{\dagger}$ in (14) and perturbing each base state variable to give:

$$
\begin{aligned}
\left\langle p^{\dagger},(\delta \mathrm{G}) p\right\rangle= & +p_{d}^{\dagger^{*}}\left(\delta \bar{v}_{d}\right) k_{d} p_{d}+p^{\dagger^{*}} \bar{v}_{d}\left(\delta k_{d}\right) p_{d}+p^{\dagger^{*}}\left(\delta \bar{v}_{u}\right) k_{u} p_{u}+p_{u}^{\dagger^{*}} \bar{v}_{u}\left(\delta k_{u}\right) p_{u} \ldots \\
& -\left\langle\frac{\mathrm{d} p^{\dagger}}{\mathrm{d} x},(\delta \bar{v}) \frac{\mathrm{d} p}{\mathrm{~d} x}\right\rangle \ldots \\
& -\left\langle p^{\dagger}, \zeta(\delta n)\left(\mathrm{e}^{-s \tau}\right) h\left\langle w_{\bar{\rho}}^{*}, \frac{\mathrm{d} p}{\mathrm{~d} x}\right\rangle\right\rangle \ldots \\
& -\left\langle p^{\dagger}, \zeta n\left(-s(\delta \tau) \mathrm{e}^{-s \tau}\right) h\left\langle w_{\bar{\rho}}^{*}, \frac{\mathrm{d} p}{\mathrm{~d} x}\right\rangle\right\rangle \ldots \\
& -\left\langle p^{\dagger}, \zeta n\left(\mathrm{e}^{-s \tau}\right)(\delta h)\left\langle w_{\bar{\rho}}^{*}, \frac{\mathrm{d} p}{\mathrm{~d} x}\right\rangle\right\rangle \ldots \\
& -\left\langle p^{\dagger}, \zeta n\left(\mathrm{e}^{-s \tau}\right) h\left\langle\left(\delta w_{\bar{\rho}}^{*}\right), \frac{\mathrm{d} p}{\mathrm{~d} x}\right\rangle\right\rangle
\end{aligned}
$$

(Note that the upstream boundary terms are positive because the upstream surface normal points in the negative $x$-direction.) If the base state perturbation, $\delta b$ or $\delta k$, is known a priori then the eigenvalue shift, $\delta s$, is found by substituting $\delta b$ or $\delta k$ into (35). If the base state perturbation is not known a priori then it is useful instead to define the sensitivities $\partial s / \partial k$ and $\partial s / \partial b(x)$ such that:

$$
\begin{aligned}
\frac{\partial s}{\partial k} \delta k & \equiv \lim _{0 \rightarrow 0} \frac{s(k+\epsilon \delta k)-s(k)}{\epsilon} \equiv \delta s \\
\int_{0}^{1} \frac{\partial s}{\partial b}(x) \delta b(x) \mathrm{d} x & \equiv \lim _{0} \frac{s(b(x)+\epsilon \delta b(x))-s(b(x))}{\epsilon} \equiv \delta s
\end{aligned}
$$

These sensitivities give the influence of $\delta k$ at the boundary and $\delta b(x)$ at every point in space. They are defined without complex conjugation, unlike the inner product (11), so that the 
corresponding Matlab code is easier to read. Equating (36) and (37) with the relevant terms in (35) leads to the algebraic expressions for the base state sensitivities in the top-left quadrant of table VII. This process also gives the sensitivity to local density changes at the boundary but this has little relevance and is not recorded here. These sensitivities are calculated with the Matlab code fun_ds_CA.m and are the same for all four discretizations. They are shown in figure 3 for the four discretizations (FDS_CA, FEW_CA, FDW_CA, SBP_CA).

\section{B. Base state sensitivities in the discrete framework}

In the discrete framework, all boundary conditions are embedded within the matrix $\mathbf{G}$, so do not need to be considered separately. A similar analysis to (31-32), but for matrices with left and right eigenvectors, $\mathbf{p}^{\dagger}$ and $\mathbf{p}$, gives:

$$
\delta s=\frac{\mathbf{p}^{\dagger^{H}} \delta \mathbf{G} \mathbf{p}}{\alpha} \quad \text { where } \quad \alpha \equiv \mathbf{p}^{\dagger^{H}}\left(\frac{\mathrm{d} \mathbf{G}}{\mathrm{d} s}\right) \mathbf{p} .
$$

In the discrete framework the base state perturbation is a column vector $\delta \mathbf{b}$ and the sensitivity of $s$ is defined as the row vector $\partial s / \partial \mathbf{b}$ such that

$$
\delta s=(\partial s / \partial \mathbf{b}) \delta \mathbf{b}
$$

For the discretized equations in the strong form (13), the sensitivities with respect to the base state variables are given in the top-right quadrant of table VII and are coded into mat_AC_FDS_DA.m. For the discretized equations in the weak form (15), the sensitivities with respect to the base state variables are given in the bottom-left quadrant of table VII and are coded into mat_AC_FEW_DA.m and mat_AC_FDW_DA.m. For the discretized equations in the strong form using a summation by parts formula with a simultaneous approximation term, the sensitivities re given in the bottom-right quadrant of table VII and are coded into mat_AC_SBP_DA.m. They are plotted in figure 3 .

In the Finite Element framework, the base state sensitivities are the same types of function as their respective base state variable. Therefore $\partial s / \partial \mathbf{h}_{1}$ and $\partial s / \partial \tau_{1}$ are $\mathrm{P} 1$ while $\partial s / \partial \mathbf{w}_{\bar{\rho} 0}$ and $\partial s / \partial \mathbf{v}_{0}$ are P0. For the weak form equations and the strong form equations in SBP-SAT form, $\bar{v}_{u} k_{u}$ is added to the bottom-right element and $\bar{v}_{d} k_{d}$ is added to the top-left element of A. The corresponding sensitivities to $k_{u}$ and $k_{d}$ are given in Table VII. For the strong form equations with replacement boundary conditions, a Robin upstream boundary condition is 
imposed by replacing the bottom row of $\mathbf{A}$ with the bottom row of $\mathbf{D}$, then adding $k_{u}$ to the bottom-right element, and multiplying the whole row by an arbitrary constant, $c_{u}$. For the downstream boundary condition, a similar procedure is used but $k_{d}$ is subtracted from the top-left element and the arbitrary constant is $c_{d}$. The inclusion of $c_{u}$ and $c_{d}$ in $\partial s / \partial k_{u}$ and $\partial s / \partial k_{d}$ means that $\partial s / \partial k_{u}$ and $\partial s / \partial k_{d}$ are calculated exactly (as shown in figure 3 ), even though the boundary values of the left eigenvector, $\mathbf{p}_{u}^{\dagger}$ and $\mathbf{p}_{d}^{\dagger}$, depend on the arbitrary constants $c_{u}$ and $c_{d}$.

\section{Problems with the base state sensitivities in the discrete strong form}

Examination of the expressions in Table VII reveals subtle features of the sensitivities derived from the discretized strong form equations with replacement boundary conditions FDS_DA. The first feature is that, although $\delta \overline{\mathbf{v}}$ and $\delta \mathbf{w}_{\bar{\rho}}$ contain $N+1$ independent elements, their corresponding sensitivities, $\partial s / \partial \overline{\mathbf{v}}$ and $\partial s / \partial \mathbf{w}_{\bar{\rho}}$, contain only $N$ independent elements. This can be seen in Table VII: the matrix $\mathbf{D}$ represents $\mathrm{d} / \mathrm{d} x$ and therefore has $\operatorname{rank} N$, meaning that $\partial s / \partial \overline{\mathbf{v}}$ is element by element multiplication of two row vectors containing $N$ independent elements, and $\partial s / \partial \mathbf{w}_{\bar{\rho}}$ is the product of a scalar with a vector $(\mathbf{M D p})^{T}$ containing $N$ independent elements. This does not cause any problems and is analogous to the fact that, in the finite element formulation, $\partial s / \partial \overline{\mathbf{v}}_{0}$ and $\partial s / \partial \mathbf{w}_{\bar{\rho} 0}$ are $\mathrm{P} 0$ functions.

The second feature is due to the fact that, for non-Dirichlet boundary conditions in the FDS_DA framework, the left eigenvector, $\mathbf{p}^{\dagger}$, oscillates, as explained in $§ I V B$ and shown in the bottom frames of figure 2. For $\partial s / \partial \tau, \partial s / \partial \mathbf{h}$, and $\partial s / \partial \overline{\mathbf{v}}$, which contain $\mathbf{p}^{\dagger}$ outside an inner product, these oscillations propagate into the sensitivities. The dependence on $c_{u}$ and $c_{d}$ disappears, however, because they appear in both the numerator and denominator of (38). The supplementary file Sup_002.m plots the FDS_DA base state sensitivities at different values of $c_{u}$, confirming that the oscillations persist but do not depend on $c_{u}$. These oscillations do not appear in $\partial s / \partial \mathbf{w}_{\bar{\rho}}$, because, for this sensitivity, $\mathbf{p}^{\dagger}$ is multiplied by $\mathbf{h}$ within an inner product and is then applied equally to every point in space.

The third feature is an extension of the second and is due to $\mathbf{p}^{\dagger}{ }^{H} \mathbf{M D}$ in the $\partial s / \partial \overline{\mathbf{v}}$

term. For reasons described in $\S \mathrm{IV} B$, the action of $\mathbf{p}^{\dagger^{H}}$ on $\mathbf{D}$ for non-Dirichlet boundary conditions creates oscillations in $\partial s / \partial \overline{\mathbf{v}}$. The fourth feature is due to the way that the boundary conditions are implemented in FDS. The top and bottom rows of $\mathbf{A}$ are replaced 
with the boundary conditions, which removes the influence of the boundary values of $\mathbf{h}$ and $\tau$ and forces the corresponding sensitivity to be zero at the boundaries.

These problems can all be avoided by using the continuous adjoint method, the discretized strong SBP-SAT form or one of the discretized weak form methods.

\section{The relationship between the continuous and the discrete frameworks}

In the continuous framework (37) the sensitivity $\partial s / \partial b$ is calculated with respect to variations in a continuous function $\delta b$. In the discrete framework (39) the sensitivity $\partial s / \partial \mathbf{b}$ is calculated with respect to variations at a gridpoint, $\delta \mathbf{b}$. The two are related by equating (37) with (39): $\delta s=\int(\partial s / \partial b) \delta b \mathrm{~d} x=(\partial s / \partial \mathbf{b}) \delta \mathbf{b}$. If $(\partial s / \partial \mathbf{b})_{\text {cont }}$ is defined to be a row vector containing the value of $(\partial s / \partial b)$ at the gridpoints $x_{i}$ then, for arbitrary $\delta \mathbf{b}$,

$$
(\partial s / \partial \mathbf{b})_{c o n t} \mathbf{M}=(\partial s / \partial \mathbf{b})
$$

Equivalently, the value of the continuous sensitivity at a gridpoint, $(\partial s / \partial \mathbf{b})_{\text {cont }}$, is given by $(\partial s / \partial \mathbf{b}) \mathbf{M}^{-1}$. When dimensional, the dimensions of $(\partial s / \partial \mathbf{b})$ are $[s][b]^{-1}$, while the dimensions of $(\partial s / \partial \mathbf{b})_{\text {cont }}$ are $[s][b]^{-1} \mathrm{~L}^{-\Delta}$, where $\Delta$ is the spatial dimension of the problem $(1,2$, or 3$)$. The Matlab function fun_Helm.m returns $(\partial s / \partial \mathbf{b})$ when the discrete adjoint is requested and $(\partial s / \partial \mathbf{b})_{\text {cont }}$ when the continuous adjoint is requested.

\section{E. Propagating sensitivities through the active iteration method}

In the active iteration method, the nonlinear eigenvalue problem (12) is solved as a sequence of linear eigenvalue problems, as described in $§ I I I E$. At the $j^{\text {th }}$ iteration, the operator $\mathrm{A}$ in (12) is labelled $\mathrm{A}_{j}$ and depends on $s_{j-1}$ through the time delay term $\mathrm{e}^{s_{j-1} \tau}$ and the Robin boundary conditions $k_{u}\left(s_{j-1}\right)$ and $k_{d}\left(s_{j-1}\right)$. The change in the operator $\mathrm{A}_{j}$ is therefore caused not only by a perturbation to the base state variables at that iteration, but also by the shift in the eigenvalue at the previous iteration, acting through $\left(\mathrm{e}^{s_{j-1} \tau}\right), k_{u}$, and $k_{d}$. To obtain the base state sensitivity exactly, this eigenvalue shift must be propagated 
through the iterations. The eigenvalue shift at the $j^{\text {th }}$ iteration is given by:

$$
\begin{aligned}
\delta s_{j} & =\left.\int_{0}^{1} \frac{\partial s_{j}}{\partial b(x)}\right|_{s_{j-1}} \delta b(x) \mathrm{d} x+\left.\frac{\partial s_{j}}{\partial s_{j-1}}\right|_{b(x)} \delta s_{j-1} \\
\text { where }\left.\quad \frac{\partial s_{j}}{\partial s_{j-1}}\right|_{b(x)} & =\frac{1}{2 s_{j}}\left(\frac{\left\langle p_{j}^{\dagger},(\partial \mathrm{A} / \partial s)_{j-1} p_{j}\right\rangle}{\left\langle p_{j}^{\dagger}, \mathrm{C} p_{j}\right\rangle}\right) \equiv \xi_{j} .
\end{aligned}
$$

The eigenvalue shift at the final iteration, $\delta s_{J}$, is found by applying (41) recursively back to $\delta s_{0}$, which is zero:

$$
\delta s_{J}=\left.\sum_{j=1}^{J} \chi_{j} \int_{0}^{1} \frac{\partial s_{j}}{\partial b(x)}\right|_{s_{j-1}} \delta b(x) \mathrm{d} x \quad \text { with } \quad \chi_{j} \equiv \begin{cases}1 & \text { for } j=J \\ \prod_{j}^{J-1} \xi_{j} & \text { for } j=1 \text { to } J-1\end{cases}
$$

Moving the summation into the integral gives the base state sensitivity of $s_{J}$ in terms of the base state sensitivities of each iteration $s_{j}$, which are listed in table VII:

$$
\frac{\partial s_{J}}{\partial b(x)}=\left.\sum_{j=1}^{J} \chi_{j} \frac{\partial s_{j}}{\partial b(x)}\right|_{s_{j-1}}
$$

The quantity $\chi_{j}$, which can be calculated during the forward iteration, gives the influence of the $j^{\text {th }}$ iteration on the final eigenvalue $s_{J}$. For a well-converged solution, $\chi_{J}$ is much greater than all the other $\chi_{j}$ and, to a good approximation, $\delta s_{J}$ is given by $\delta s_{j}$ at the final iteration. This is of practical interest because it reduces the number of direct and adjoint eigenfunctions that need to be stored in order to obtain an accurate base state sensitivity. Another convenient point is that the discrete adjoint codes can be debugged ( $\S \mathrm{VF}$ ) by considering just two iterations (i.e. before convergence), which speeds up code development.

\section{F. Debugging the discrete base state sensitivities with a Taylor test}

The eigenvalue shift, $\delta s$, for a given base state, $\delta b$, can be calculated via finite difference: $\delta s=s(b+\delta b)-s(b)$. If the eigenvalue is analytic with respect to the perturbation, this eigenvalue shift can also be expressed as a Taylor expansion:

$$
\delta s=\frac{\partial s}{\partial b} \delta b+\frac{1}{2} \frac{\partial^{2} s}{\partial b^{2}}(\delta b)^{2}+\mathcal{O}(\delta b)^{3}
$$

In the discrete framework, the left and right eigenvectors, $\mathbf{p}^{\dagger}$ and $\mathbf{p}$, are calculated to machine precision from the same matrix. The first order sensitivity, $\partial s / \partial \mathbf{b}$, in (39) is therefore calculated to machine precision. The difference between $\delta s$ calculated with a finite difference 
method and $\delta s$ calculated with the adjoint method must therefore increase in proportion to $(\delta b)^{2}$ and higher orders. If it increases in proportion to $\delta b$ then there is a bug in the code. This is a stringent and helpful test for debugging. This is implemented in the Matlab function fun_TT.m.

\section{FEEDBACK SENSITIVITY}

In this section, the feedback sensitivity is defined (§VI A) and then the theory and numerics are described for the continuous framework (§VIB), and the discrete framework (§VIC), in the FDS, FEW, FDW, and SBP formulations. The physical interpretation of the feedback sensitivities is in $\S$ VIII B.

\section{A. Linear modelling of passive feedback devices}

All feedback devices can be modelled in terms of their feedback from $u$ and/or $p$ into the mass, momentum and/or energy equations. The feedback can be local in space and time, which would be typical for control via a passive device, or non-local, which would be typical for feedback control with a non-colocated sensor and actuator. In this paper, only local

feedback will be considered, described by the functions $\delta \dot{m}_{\bar{\rho}, u}, \delta \dot{m}_{\bar{\rho}, p}, \delta \mathbf{f}_{\bar{\rho}, u}, \delta \mathbf{f}_{\bar{\rho}, p}, \delta \dot{q}_{\bar{p}, u}, \delta \dot{q}_{\bar{p}, p}$, such that the feedback terms in (10) are local linear functions of $u$ and $p$ :

$$
\begin{gathered}
\delta \dot{m}_{\bar{\rho}}=\delta \dot{m}_{\bar{\rho}, u} u+\delta \dot{m}_{\bar{\rho}, p} p \\
\delta \mathbf{f}_{\bar{\rho}}=\delta \mathbf{f}_{\bar{\rho}, u} u+\delta \mathbf{f}_{\bar{\rho}, p} p \\
\delta \dot{q}_{\bar{p}}=\delta \dot{q}_{\bar{p}, u} u+\delta \dot{q}_{\bar{p}, p} p .
\end{gathered}
$$

The change, $\delta \mathrm{A}$, to the linear operator $\mathrm{A}$ in $(12 \mathrm{~b})$ is found by substituting (46) into (10). 


\section{B. Feedback sensitivity in the continuous framework}

The feedback causes a change, $\delta \mathrm{A}$, in the operator (12b). The sensitivity of the eigenvalue, $s$, to this change is found from the numerator of (33), noting that $\delta \mathrm{G}=\delta \mathrm{A}$ :

$$
\begin{aligned}
\left\langle p^{\dagger},(\delta \mathrm{A}) p\right\rangle & =\left\langle p^{\dagger}, s \delta \dot{m}_{\bar{\rho}, u} u\right\rangle+\left\langle p^{\dagger}, s \delta \dot{m}_{\bar{\rho}, p} p\right\rangle \\
\ldots+ & \left.\ldots \frac{\mathrm{d}_{p^{\dagger}}}{\mathrm{d} x}, \delta \mathbf{f}_{\bar{\rho}, u} u\right\rangle+\left\langle\frac{\mathrm{d} p^{\dagger}}{\mathrm{d} x}, \delta \mathbf{f}_{\bar{\rho}, p} p\right\rangle-\left[p^{\dagger} \delta \mathbf{f}_{\bar{\rho}, u} u+p^{\dagger} \delta \mathbf{f}_{\bar{\rho}, p} p\right]_{0}^{1} \ldots \\
\ldots+\left\langle p^{\dagger}, s \zeta \delta \dot{q}_{\bar{p}, u} u\right\rangle+\left\langle p^{\dagger}, s \zeta \delta \dot{q}_{\bar{p}, p} p\right\rangle & .
\end{aligned}
$$

In (47), the $\delta \mathbf{f}_{\bar{\rho}, u}$ and $\delta \mathbf{f}_{\bar{\rho}, p}$ terms have been integrated by parts. Their boundary terms can be calculated but are of little value and are dropped from the subsequent analysis. The eigenvalue shift can be found if the feedback mechanism is known a priori. It is more useful, however, to obtain the influence of local feedback at every point in space, $\partial s / \partial l(x)$, defined

such that $\delta s \equiv \int(\partial s / \partial l) \delta l \mathrm{~d}(x)$, where $\delta l$ represents the functions $\delta \dot{m}_{\bar{\rho}, u}, \delta \dot{m}_{\bar{\rho}, p}, \delta \mathbf{f}_{\bar{\rho}, u}, \delta \mathbf{f}_{\bar{\rho}, p}$, $\delta \dot{q}_{\bar{p}, u}$, or $\delta \dot{q}_{\bar{p}, p}$. The feedback sensitivities are listed in Table VIII and are shown in figure 4 (FDS_CA, FEW_CA, FDW_CA, SBP_CA). These four lines all lie on top of each other, showing that there are no numerical problems when deriving the feedback sensitivities in the continuous framework.

\section{Feedback sensitivity in the discretized strong form equations}

For the finite difference method applied to the strong form $\S$ III A, the feedback causes a change, $\delta \mathbf{A}$, in the matrix $\mathbf{A}(13 \mathrm{~b})$ such that:

$$
\begin{array}{r}
\delta \mathbf{A}=\quad s \mathbf{M}\left(\delta \dot{\mathbf{M}}_{\bar{\rho}, u}\right) \mathbf{U}+s \mathbf{M}\left(\delta \dot{\mathbf{M}}_{\bar{\rho}, p}\right) \ldots \\
\ldots-\operatorname{MD}\left(\delta \mathbf{F}_{\bar{\rho}, u}\right) \mathbf{U}-\mathbf{M D}\left(\delta \mathbf{F}_{\bar{\rho}, p}\right) \ldots \\
\ldots+s \zeta \mathbf{M}\left(\delta \dot{\mathbf{Q}}_{\bar{p}, u}\right) \mathbf{U}+s \zeta \mathbf{M}\left(\delta \dot{\mathbf{Q}}_{\bar{p}, p}\right)
\end{array}
$$

where $\mathbf{U} \equiv-\mathbf{V D} / s$.

and where $\delta \dot{\mathbf{M}}_{\bar{\rho}, u}$ is a matrix containing the values of $\delta \dot{m}_{\bar{\rho}, u}\left(x_{i}\right)$ along the diagonal. (The other $\delta$ matrices are similarly defined.) The eigenvalue shift is then given by (38) with $\delta \mathbf{G}=\delta \mathbf{A}$. The feedback sensitivity in the discrete framework, $(\delta s / \delta \mathbf{l})$, is a row vector such that $\delta s=(\partial s / \partial \mathbf{l}) \delta \mathbf{l}$. The value of the continuous sensitivity at a gridpoint point, $(\partial s / \partial \mathbf{l})_{\text {cont }}$, is given by $(\partial s / \partial \mathbf{l}) \mathbf{M}^{-1}$. These feedback sensitivities are listed in table VIII and shown in figure 4 (FDS_DA). The problems described in $\S \mathrm{V} \mathrm{C}$ carry through to the feedback sensitivities. 


\section{Feedback sensitivity in the Finite Element framework}

In order to implement the feedback terms in the finite element framework, it is necessary to consider whether each local feedback function, $\delta l(x)$, should be a P0 or P1 function. The direct and adjoint eigenfunctions, $p$ and $p^{\dagger}$, are P1 functions, while $u$ and $\mathrm{d} p^{\dagger} / \mathrm{d} x$ are P0 functions. In (47), the inner products containing $\delta \dot{m}_{\bar{\rho}, p}$ and $\delta \dot{q}_{\bar{p}, p}$ are formed between two P1 functions, so $\delta \dot{m}_{\bar{\rho}, p}$ and $\delta \dot{q}_{\bar{p}, p}$ are P1 functions. Similarly, the inner product containing $\delta \mathbf{f}_{\bar{\rho}, u}$ is formed between two $\mathrm{P} 0$ functions, so $\delta \mathbf{f}_{\bar{\rho}, u}$ is a $\mathrm{P} 0$ function. The remaining three inner products contain both $\mathrm{P} 0$ and $\mathrm{P} 1$ functions and, because inner products can be formed between $\mathrm{P} 0$ and $\mathrm{P} 1$ functions using the $\mathbf{M}_{01}$ matrix, one could define $\delta \dot{m}_{\bar{\rho}, u}, \delta \mathbf{f}_{\bar{\rho}, p}$, and $\delta \dot{q}_{\bar{p}, u}$ to be either P0 or P1 functions. Their corresponding feedback sensitivities, $\partial s / \partial \dot{m}_{\bar{\rho}, u}, \partial s / \partial \mathbf{f}_{\bar{\rho}, p}$, and $\partial s / \partial \dot{q}_{\bar{p}, u}$, however, have to be $\mathrm{P} 0$ functions. This is because $\mathbf{M}_{01}$ has rank $N$ and its application to a P1 function removes one degree of freedom. Consequently, the feedback sensitivities are element by element multiplication of two row vectors with $N$ degrees of freedom and have to be P0 functions. It is then convenient (although not essential) to define $\delta \dot{m}_{\bar{\rho}, u}, \delta \mathbf{f}_{\bar{\rho}, p}$, and $\delta \dot{q}_{\bar{p}, u}$ to be $\mathrm{P} 0$ functions as well. With these definitions, the feedback causes a change $\delta \mathbf{A}_{11}$ in the matrix $\mathbf{A}_{11}$ in (15b) such that:

$$
\begin{aligned}
& \delta \mathbf{A}_{11}=\mathbf{M}_{10} s\left(\delta \dot{\mathbf{M}}_{\bar{\rho}, u, 00}\right) \mathbf{U}_{00}+\mathbf{M}_{11} s\left(\delta \dot{\mathbf{M}}_{\bar{\rho}, p, 11}\right) \quad \ldots \\
& \ldots+\mathbf{D}_{01}^{H} \mathbf{M}_{00}\left(\delta \mathbf{F}_{\bar{\rho}, u, 00}\right) \mathbf{U}_{00}+\mathbf{D}_{01}^{H} \mathbf{M}_{00}\left(\delta \mathbf{F}_{\bar{\rho}, p, 00}\right) \overline{\mathbf{M}}_{01} \ldots \\
& \ldots+\mathbf{M}_{10} s \zeta\left(\delta \dot{\mathbf{Q}}_{\bar{p}, u, 00}\right) \mathbf{U}_{00}+\mathbf{M}_{11} s \zeta\left(\delta \dot{\mathbf{Q}}_{\bar{p}, p, 11}\right) \quad \text {, }
\end{aligned}
$$

where $\mathbf{U}_{00} \equiv-\mathbf{V}_{00} \mathbf{D}_{01} / s$.

There is a neater way to write the $\delta \mathbf{F}_{\bar{\rho}, p, 00}$ term in (49). The matrices $\mathbf{M}_{00}$ and $\delta \mathbf{F}_{\bar{\rho}, p, 00}$ are both diagonal so their order can be swapped. Then $\mathbf{M}_{00} \overline{\mathbf{M}}_{01}$ can be replaced with $\mathbf{M}_{01}$, as described in $\S$ III B. The term then becomes $\mathbf{D}_{01}^{H} \delta \mathbf{F}_{\bar{\rho}, p, 00} \mathbf{M}_{01}$. These feedback sensitivities are listed in table VIII and shown in figure 4 (FEW_DA). The weak form of the finite difference framework is identical (FDW_DA). These lines lie on top of the other sensitivities, showing that there are no numerical problems when the equations are expressed in the weak form.

\section{E. Feedback sensitivity in the SBP-SAT framework}

The feedback sensitivities for the finite difference method applied in the strong SBP-SAT form are a combination of the FDS and FEW forms. They are shown in table VIII and plotted 
in figure 4 (SBP_DA). There are no numerical problems for this method.

\section{RECEPTIVITIES}

A general approach to receptivity via adjoint equations, which is independent of the solution method, can be found in [36, §3.1.1] [37, p. 10], [11], and [38, §2.5]. The most relevant result from [38] is for constant amplitude forcing of a stable system. The system in this paper is forced with periodic injection of $\dot{m}_{\bar{\rho}}(x) \mathrm{e}^{s_{f} t}$ into the mass equation (2c), $\mathbf{f}_{\bar{\rho}}(x) \mathrm{e}^{s_{f} t}$ into the momentum equation $(2 \mathrm{~d})$, and $\dot{q}_{\bar{p}}(x) \mathrm{e}^{s_{f} t}$ into the energy equation (2e), where $s_{f}$ is an imaginary number equal to the angular frequency of forcing. Ref. [38, eq. (17)] shows that the system's linear response has contributions from all eigenfunctions, $p_{k}$, and is at the forcing frequency, $s_{f}$. For mass injection, the amplitude of the contribution from eigenfunction $p_{k}$ is proportional to $\left\langle p^{\dagger}{ }_{k} s^{*}, \dot{m}_{\bar{\rho}}\right\rangle /\left(s_{f}-s_{k}\right)$, where $s_{k}$ is the eigenvalue of $p_{k}$. The contribution from eigenfunction $p_{k}$ is therefore greater (i) the closer $s_{f}$ is to $s_{k}$ and (ii) the more the spatial structure of the forcing, $\dot{m}_{\bar{\rho}}$, projects onto the function $p_{k}^{\dagger} s^{*}$, which is labelled the receptivity, $\dot{m}_{\bar{\rho}, k}^{\dagger}(x)$. (This receptivity is the term to the left of $u$ in the expression for $\left(\partial s / \partial \dot{m}_{\bar{\rho}, u}\right)$ in table VIII.) Similarly, the expressions for the receptivities to momentum and heat injection are $\mathbf{f}_{\bar{\rho}, k}^{\dagger}=\left(\mathrm{d} p_{k}^{\dagger} / \mathrm{d} x\right)$, and $\dot{q}_{\bar{p}, k}^{\dagger}=p_{k}^{\dagger} s^{*} \zeta$.

Ref. [38, eq. 18] also shows that, for constant amplitude forcing of a system with one unstable eigenmode, $k$, the system's linear response has frequency/growth rate, $s_{k}$, and spatial structure $p_{k}$. As before, the magnitude of the response is greater (i) the closer $s_{f}$ is to $s_{k}$ and (ii) the more the spatial structure of the forcing, $\dot{m}_{\bar{\rho}}$, projects onto the receptivity $\dot{m}_{\bar{\rho}, k}^{\dagger}(x)$.

The absolute values of the receptivities $\left|\dot{m}_{\bar{\rho}}^{\dagger}\right|,\left|\mathbf{f}_{\bar{\rho}}^{\dagger}\right|$, and $\left|\dot{q}_{\bar{p}}^{\dagger}\right|$, are shown in figure 5 for the dominant mode of the Rijke tube and rocket engine models. These show where the system is most receptive to open loop forcing. This will be interpreted physically in $\S$ VIII A. As expected, there are no numerical problems for the continuous adjoints (*_CA) and for the discrete adjoints constructed from the weak form (FEW_DA and FDW_DA) but there are severe oscillations for the discrete adjoints constructed from the strong form with replacement boundary conditions (FDS_DA). 


\section{PHYSICAL INTERPRETATION}

\section{A. Receptivities}

The most convenient starting point for physical interpretation are the receptivities $\left|\dot{m}_{\bar{\rho}}^{\dagger}\right|$, $\left|\mathbf{f}_{\bar{\rho}}^{\dagger}\right|$, and $\left|\dot{q}_{\bar{p}}^{\dagger}\right|$ in figure 5. Using the relations in (6), the sensitivity of the eigenvalue to generic periodic injections of mass into the mass equation, $\dot{m}^{\dagger}$, equals $\dot{m}_{\bar{\rho}}^{\dagger} / \bar{\rho}$. Similarly, $\mathbf{f}^{\dagger}=\mathbf{f}_{\bar{\rho}}^{\dagger} / \bar{\rho}$ and $\dot{q}^{\dagger}=\dot{q}_{\bar{p}}^{\dagger} / \bar{p}$. Table VIII shows that the receptivity of the energy equation, $\dot{q}^{\dagger}$, is proportional to the adjoint pressure eigenfunction, $p^{\dagger}$. In other words, extra heat addition has most influence on the eigenvalue when it is applied to regions in which the adjoint pressure is highest. This can be anticipated from the fact that the adjoint equation (23b) is forced with a term proportional to $\left\langle\zeta n\left(\mathrm{e}^{-s \tau}\right) h, p^{\dagger}\right\rangle$. In the self-adjoint case, this becomes equivalent to Rayleigh's criterion [3]. The receptivity of the mass equation, $\dot{m}^{\dagger}$, is proportional to $\bar{v} p^{\dagger}$. This is similar to $\dot{q}^{\dagger}$ but includes the fact that mass injection has most influence in regions of lower density.

The receptivity of the momentum equation, $\mathbf{f}^{\dagger}$, is proportional to $\bar{v} \mathrm{~d} p^{\dagger} / \mathrm{d} x$, which has a clear physical explanation. The heat release term in the adjoint equation (23b) acts in the measurement region (where $\mathrm{d} w_{\bar{\rho}}^{*} / \mathrm{d} x \neq 0$ ), which results in $\mathrm{d} p^{\dagger} / \mathrm{d} x$ being largest in this region (figure 2). This term therefore reveals that momentum injection has most influence in the measurement region, the more so when the density is smaller ( $\bar{v}$ larger) because momentum injection will then cause a larger change in velocity. This describes the unsurprising feature that the eigenvalue is strongly receptive to interference with the velocity in the measurement region.

\section{B. Feedback sensitivities}

The feedback sensitivities in figure 6 show the real and imaginary components of the absolute values of $|\partial s / \partial l|$ shown in figure 4. These are the products of the direct eigenfunctions, $p$ and $u$ (figure 1), with the receptivities, $\dot{m}_{\bar{\rho}}^{\dagger}, \mathbf{f}_{\bar{\rho}}^{\dagger}$, and $\dot{q}_{\bar{p}}^{\dagger}$ (figure 5). The real component of $\partial s / \partial l$ is the influence of local in-phase feedback on the growth rate (positive $=$ destabilizing). The imaginary component is the influence on the frequency.

For the Rijke tube, which is nearly self-adjoint, the real components (thin dark red lines) of $\partial s / \partial \dot{m}_{\bar{\rho}, p}, \partial s / \partial \mathbf{f}_{\bar{\rho}, u}$, and $\partial s / \partial \dot{q}_{\bar{p}, p}$, are large and positive. This shows that if mass 
injection is in phase with pressure, or if momentum injection is in phase with velocity or if energy injection is in phase with pressure (and hence temperature) then the growth rate of oscillations will increase. The most influential positions of such devices are the high amplitude regions in figure 6 . This is a re-statement of page 226 of Chu [4]: "if $m^{\prime}$ [mass injection] is in phase with $p^{\prime}$ [acoustic pressure], or $F^{\prime}$ [momentum injection] with $u^{\prime}$ [acoustic velocity] or $Q^{\prime}$ [fluctuating heat release rate] with $T^{\prime}$ [acoustic temperature] then energy will be continuously fed into the disturbance".

For the rocket engine, which is strongly non-self-adjoint, the real components (thin dark red lines) of $\partial s / \partial \dot{m}_{\bar{\rho}, p}, \partial s / \partial \mathbf{f}_{\bar{\rho}, u}$, and $\partial s / \partial \dot{q}_{\bar{p}, p}$, are large, but not always positive. Upstream of the measurement region $\left(X_{w}=0.06\right)$, the growth rate of oscillations will decrease if mass injection is in phase with pressure, if momentum injection is in phase with velocity, or if heat injection is in phase with temperature. This differs from Chu's statement [4] because, as shown in $\S \mathrm{VII}$, the receptivities of the mass, momentum, and energy equations need to be formed with the adjoint pressure, $p^{\dagger}$, rather than the direct pressure, $p$. For the rocket, $p^{\dagger}$ differs considerably from $p$. For comparison, the feedback sensitivities when the receptivities are formed with the direct pressure, $p$, are also shown in Fig. 6 (thick light blue and red lines). The real components (thick light red lines) of these (incorrect) versions of $\partial s / \partial \dot{m}_{\bar{\rho}, p}$, $\partial s / \partial \mathbf{f}_{\bar{\rho}, u}$, and $\partial s / \partial \dot{q}_{\bar{p}, p}$, are always positive, as expected from Chu's statement. Further, for the Rijke tube, they are almost identical to the correct feedback sensitivities because the Rijke tube is nearly self-adjoint. This conclusion carries over into the Rayleigh criterion, which states that the acoustic energy grows over a cycle if $\oint p q \mathrm{~d} t$ exceeds the damping. In a linear stability analysis, $p$ in the Rayleigh criterion should be replaced with the receptivity of the energy equation, $\dot{q}^{\dagger}$, which is proportional to the adjoint pressure, $p^{\dagger}$, rather than the direct pressure $p$. This makes a significant difference in strongly non-self adjoint systems.

\section{Base state sensitivities}

The base state sensitivities in figure 7 show the real and imaginary components of the absolute values in figure 3 . The sensitivity of the eigenvalue to changes in $h(x)$ has the shape of the adjoint pressure eigenfunction, $p^{\dagger}$, and amplitude $\zeta n \mathrm{e}^{-s \tau} \int w_{\bar{\rho}}(\mathrm{d} p / \mathrm{d} x) \mathrm{d} x$. In physical terms, the shape follows that of the receptivity of the energy equation and the amplitude is large if the measurement function, $w_{\bar{\rho}}$, is large in regions in which the velocity is large. If $\tau$ 
varies in space, then this sensitivity oscillates in space. The sensitivity to changes in $\tau(x)$ is significant only in the heat release region, $h(x)>0$. The sensitivity to changes in the measurement region, $w_{\bar{\rho}}$, has the shape of the direct velocity and amplitude $\left\langle p^{\dagger}, \zeta n \mathrm{e}^{-s \tau} h\right\rangle$. In physical terms it is proportional to the local velocity in the measurement region and to the Rayleigh integral formed with the adjoint pressure. The base state sensitivities formed by replacing the adjoint pressure $p^{\dagger}$ with the direct pressure, $p$ are shown for comparison. As expected, there is little discrepancy for the Rijke tube, which is nearly self-adjoint, but significant discrepancy for the rocket engine, which is strongly non-self adjoint.

\section{APPLICATIONS OF THE BASE STATE SENSITIVITIES}

The base state sensitivities (figure 7) show, at a glance, how the growth rate and frequency of the thermoaoustic mode are affected by changes to the base state parameters at each point in space and by changes to the boundary conditions. This is useful for physical understanding of how the thermoacoustic mechanism acts in a given system and can inform strategies to reduce thermoacoustic instability. Furthermore, these base state sensitivities can cheaply be converted into sensitivities to the design parameters. This allows a design procedure to be automated such that every unstable thermoacoustic mode can be stabilized by making small changes to the design parameters. For a network model with longitudinal waves only, this procedure is demonstrated for a laboratory burner in [16]. In this case, which was chosen for its difficulty, the seven initially unstable modes are all stabilized by making small geometry changes. For a network model with longitudinal and azimuthal waves only, this procedure is demonstrated for axisymmetric changes to an annular gas turbine combustion chamber in [17]. In this case, which was chosen for its realism, the two initially unstable modes are both stabilized by changing areas and lengths by no more than $6 \%$. Here, the procedure is demonstrated for 1D Helmholtz solvers.

\section{A. Design parameter sensitivities}

Table VII contains the base state sensitivities to the internal parameters, $\bar{v}(x), n, \tau(x), h(x), w_{\bar{\rho}}(x)$. In the corresponding Matlab codes, these internal parameters are functions of external (design) parameters, $X_{w}, L_{w}, X_{h}, L_{h}, \tau, R_{u}, R_{d}$, which are set in fun_param_dim.m. The sensi- 
tivities of the eigenvalue with respect to the external parameters is found using the chain rule. For example, the sensitivity with respect to the heat release location, $X_{h}$, keeping all other design parameters constant is:

$$
\left.\frac{\partial s}{\partial X_{h}}\right|_{X_{w} \ldots R_{d}}=\int_{0}^{1}\left(\frac{\partial s}{\partial h} \frac{\partial h}{\partial X_{h}}+\frac{\partial s}{\partial \bar{v}} \frac{\partial \bar{v}}{\partial X_{h}}+\frac{\partial s}{\partial w_{\bar{\rho}}} \frac{\partial w_{\bar{\rho}}}{\partial X_{h}}\right) \mathrm{d} x \quad \text { in the continuous form }
$$

and $\left.\frac{\partial s}{\partial X_{h}}\right|_{X_{w} \ldots R_{d}}=\frac{\partial s}{\partial \mathbf{h}} \frac{\partial \mathbf{h}}{\partial X_{h}}+\frac{\partial s}{\partial \overline{\mathbf{v}}} \frac{\partial \overline{\mathbf{v}}}{\partial X_{h}}+\frac{\partial s}{\partial \mathbf{w}_{\bar{\rho}}} \frac{\partial \mathbf{w}_{\bar{\rho}}}{\partial X_{h}} \quad$ in the discrete form .

The partial derivatives on the right hand side are returned by fun_h.m, fun_rh.m, and fun_wr.m. Some of the external parameters, such as the heat release position, are known accurately. Others, such as the heat release time delay and reflection coefficients are not. By calculating these sensitivities, the user can discover which parameters have most influence on the experimental measurements and, therefore, which need to be measured accurately. These sensitivities are also useful for rapid uncertainty quantification, as shown for a thermoacoustic network model in [39].

\section{B. Gradient-based optimization with adjoints}

In thermoacoustics, gradient-based shape optimization with adjoints has been applied via a network model in $[16,17]$. In the current paper, realistic burner geometries cannot be considered because the adjoint Helmholtz solver is one-dimensional. Nevertheless, gradientbased optimization of a Rijke tube with adjoints can be demonstrated. Figure 8 shows the non-dimensional growth rates (colour contours) and non-dimensional frequencies (black line contours) for the Rijke tube with a variable-diameter iris placed at the downstream boundary and a variable heater position. The reflection coefficient, $R_{d}$, is assumed to be a function of the iris diameter, $d_{i}$, such that $R_{d}=0.97 \cos \left(\pi d_{i}\right)+0.80 \mathrm{i} \sin \left(\pi d_{i}\right)$. (This empirical relation was taken from unpublished measurements and is used only for demonstration.) Here, the measurement point $X_{w}$ is the same as the heat release point $X_{h}$. The gradient of $s_{r}$ with

respect to $X_{h}$ and $d_{i}$, as calculated with the base state sensitivity, is shown by the white arrows. This confirms that the arrows point in the correct direction. An optimization algorithm using a BFGS quasi-Newton algorithm with an Armijo line search and a penalty function is used to converge from a starting point (light dot) to the point with the maximum growth rate at a target frequency (dark dot). In this case the target frequency is 3.2. The 
gradient information is used in the quasi-Newton method to estimate the inverse Hessian, which greatly speeds up the rate of convergence compared with other algorithms.

\section{APPLICATIONS OF THE FEEDBACK SENSITIVITIES}

The design parameter sensitivities, which are derived from the base state sensitivities, show the influence of small changes to a given design. However, they do not show the influence of a qualitative change to the design, such as adding a new component. This influence can be calculated at negligible extra cost by multiplying the feedback sensitivities by a number, as described in this section. This is useful because many industrial gas turbines are stabilized by retro-fitting passive feedback devices such as Helmholtz resonators. The techniques in this section show the best locations for such devices and, within the bounds of a linear analysis, can calculate their influence on the eigenvalues. The feedback sensitivities can also be used to estimate the influence of features such as the viscous and thermal boundary layers that exist in reality but are not included in this model.

\section{A. Calculating the influence of an adiabatic mesh}

In the experiments of [33] on the electrically heated Rijke tube being modelled in this paper, the shift in growth rate is measured when a mesh of width $\delta X$ is introduced at position $X_{m}$ (figure 9). The drag force of the mesh on the flow can be considered as local instanteneous feedback from the acoustic velocity into the acoustic momentum equation. Its influence on the growth rate and frequency can be estimated with the feedback sensitivity $\partial s / \partial \mathbf{f}_{\bar{\rho}, u}$. The pressure drop across the mesh is assumed to be $\Delta p=-K \bar{\rho}(\bar{u}+\tilde{u})^{2} / 2$, where $\bar{u}$ is the mean velocity, $\tilde{u}$ is the fluctuating velocity, and $K$ is a quasi-steady pressure drop coefficient $K$. In the experiments of [33], the mean speed $\bar{u}$ is estimated as $0.1 \mathrm{~ms}^{-1}$ and $K$ is estimated as 20, based on the ratio of free area to total area of the wire mesh [40]. The total fluctuating force is therefore $-S_{c} K \overline{\rho u} \tilde{u}$, where $S_{c}$ is the cross-sectional area of the tube. This acts over a volume $S_{c} \delta X$ so the fluctuating force per unit volume is $\delta \tilde{\mathbf{f}}=\overline{\rho u} \tilde{u} K / \delta X$. Applying the definitions (6) and (46b) and moving to the frequency domain in one spatial dimension gives $\delta \mathbf{f}_{\bar{\rho}, u}=\bar{u} K / \delta X$. Substituting into (37), assuming uniformity over $\delta X_{m}$, and 
expressing in dimensional form (table VIII with $\Delta=1$ ) gives:

$$
\delta s^{\star}=\int_{X_{m}}^{X_{m}+\delta X} \frac{1}{L_{r e f}}\left(\frac{\partial s}{\partial \mathbf{f}_{\bar{\rho}, u}}\right)^{\star} \delta \mathbf{f}_{\bar{\rho}, u} \mathrm{~d} x=\frac{\bar{u} K}{L_{r e f}}\left(\frac{\partial s}{\partial \mathbf{f}_{\bar{\rho}, u}}\left(X_{m}\right)\right)^{\star}=-2.0\left(\frac{\partial s}{\partial \mathbf{f}_{\bar{\rho}, u}}\left(X_{m}\right)\right)^{\star} .
$$

The growth rate shift predicted from (52) compares favourably with that measured experimentally in figure 10, as a function of the position of the mesh, $X_{m}$. The maximum growth rate shift of $-2 \mathrm{rad} \mathrm{s}^{-1}$ is a useful benchmark against which to compare the growth rate shifts in the next sections.

\section{B. Calculating the influence of a hot mesh}

In the experiments of [41], the shift in growth rate is measured when a hot mesh of width $\delta X$ is introduced at position $X_{m}$. The heat transfer from the mesh to the flow can be considered as local feedback from the acoustic velocity into the acoustic energy equation with a small time delay. Its influence on the growth rate and frequency can be estimated with the feedback sensitivity $\left(\partial s / \partial \dot{q}_{\bar{p}, u}\right)$. The heat transfer model is assumed to be the same as that of the main hot mesh, i.e. an $n-\tau$ model with the same time delay $\tau$, FTF, and mean

flow speed $\bar{u}$ but a different power, $\dot{Q}_{m}$. This gives $\eta_{m}=\dot{Q}_{m}|F T F| / \bar{u}$. The measurement location and heat release location are both set to $X_{m}$. The heat release per unit volume at $X_{m}$ is therefore $\delta \tilde{\dot{q}}=\eta_{m} \tilde{u} \mathrm{e}^{s \tau} /\left(S_{c} \delta X\right)$. The dimensional shift in the eigenvalue is:

$$
\delta s=\frac{U_{r e f}}{L_{r e f}} \frac{\eta_{m}}{\bar{p} S_{c}} \mathrm{e}^{s \tau}\left(\frac{\partial s}{\partial \dot{q}_{\bar{p}, u}}\left(X_{m}\right)\right)^{\star}=(-9.2-54 \mathrm{i})\left(\frac{\partial s}{\partial \dot{q}_{\bar{p}, u}}\left(X_{m}\right)\right)^{\star} .
$$

The eigenvalue shift per unit power of the secondary heater is plotted in figure 11 . This shows that the largest growth rate shift due to the heat release from the secondary hot mesh exceeds that due to the drag from the secondary hot mesh when the secondary heater power exceeds around 2.0/0.05 = 40 Watts. This is a useful rule of thumb when designing experiments. The comparison with the experimental results of [41] shows an off-set. This is due to a change in the base state caused by the secondary heater, which is not considered in the current model but which has been included in Ref. [42]. Finally, the results in this section can be checked by making the secondary hot mesh identical to the primary hot mesh, $\eta_{m}=\eta$, removing damping by setting $R_{u}=R_{d}=-1$, and checking that the growth rate shift due to the secondary hot mesh is equal to the growth rate due to the primary hot mesh when they are both in the same position. This can be checked by running Sup_003.m. 


\section{Calculating the influence of the viscous boundary layer}

The viscous acoustic boundary layer has not been included in the model. Its influence on the eigenvalue can be estimated with the feedback sensitivity as an a posteriori check that its omission from the model is justified. For a simple analysis, the fluctuating wall shear stress $\tau_{m}$ can be approximated as $-\bar{\rho} \nu \tilde{u} / \delta_{b l}$, where $\nu$ is the kinematic viscosity, $\tilde{u}$ is the free stream perturbation velocity, and $\delta_{b l}(x)$ is the local acoustic boundary layer thickness. The acoustic boundary layer thickness can be estimated from Stokes' solution for oscillatory flow at angular frequency $s_{i}$ above a stationary boundary: $\delta_{b l}=2 \pi\left(2 \nu / s_{i}\right)^{1 / 2}$. For an element of tube with length $\delta X$ and perimeter $\Gamma_{c}$, the total fluctuating force is therefore $-\Gamma_{c} \delta X \bar{\rho} \nu \tilde{u} / \delta_{b l}$. Following the same analysis as that in $\S \mathrm{X}$ A gives the local eigenvalue shift per unit length of the boundary layer:

$$
\frac{\delta s}{\delta X}=-\frac{1}{L_{r e f}} \frac{\Gamma}{S_{c}} \frac{\nu}{\delta_{b l}}\left(\frac{\partial s}{\partial \mathbf{f}_{\bar{\rho}, u}}(X)\right)^{\star}=1.26\left(\frac{\partial s}{\partial \mathbf{f}_{\bar{\rho}, u}}(X)\right)^{\star} .
$$

The results are shown in figure 12 , showing that the total decay rate due to the viscous boundary layer, integrated along the length of the chamber, is approximately $-0.61 \mathrm{rad} \mathrm{s}^{-1}$. The local decay rate is largest at the ends of the tube, where the acoustic speed is largest. For comparison, the decay rate due to acoustic radiation from the tube is around $-10 \mathrm{rad}$ $\mathrm{s}^{-1}$. (This can be calculated by setting $\dot{Q}_{h}=0$ in fun_param_dim.m.) The decay due to the viscous boundary layer is therefore around $6 \%$ of that due to acoustic radiation from the open ends of the tube, which is sufficiently small to be neglected from the current analysis.

\section{Calculating the influence of the thermal boundary layer}

Similarly, the influence of the thermal boundary layer can be calculated with the feedback sensitivity. The gas temperature is $\bar{T}+\tilde{T}$. The wall is assumed to be in thermal equilibrium with the local mean flow at $\bar{T}$. The heat transfer into the gas from an element of tube with length $\delta X$ and perimeter $\Gamma_{c}$ is $\delta \tilde{\dot{q}}=-\left(\Gamma_{c} \delta X\right)\left(\lambda / \delta_{b l}\right) \tilde{T}$, where $\delta_{b l}$ is the local thermal boundary layer thickness and $\lambda=\bar{\rho} \nu c_{p} / \operatorname{Pr}$. The acoustic thermal boundary layer thickness is taken to be the same as the acoustic momentum boundary layer thickness. The Prandtl number is set to $\operatorname{Pr}=0.7$ and, by construction, $P_{\text {ref }}=\bar{p}$. Sound waves are isentropic so

$(\tilde{T} / \bar{T})=(\tilde{p} / \bar{p})(\gamma-1) / \gamma$. Using the ideal gas law, $\bar{p}=\bar{\rho} r_{g} \bar{T}$, the above expressions can be combined to obtain $\delta \tilde{\dot{q}}=-\Gamma_{c} \delta X \nu \tilde{p} /\left(\operatorname{Pr} \delta_{b l}\right)$. The thermal boundary layer is therefore 
modelled as a feedback term from the pressure into the energy equation. Following analysis similar to that in $\S \mathrm{X} \mathrm{C}$ gives the local eigenvalue shift per unit length of the boundary layer:

$$
\frac{\delta s}{\delta X}=-\frac{P_{r e f}}{L_{r e f}} \frac{\Gamma_{c}}{S_{c}} \frac{\nu}{\delta_{b l}} \frac{1}{\operatorname{Pr}} \frac{1}{\bar{p}}\left(\frac{\partial s}{\partial \dot{q}_{\bar{p}, p}}(X)\right)^{\star}=1.79\left(\frac{\partial s}{\partial \dot{q}_{\bar{p}, p}}(X)\right)^{\star} .
$$

This produces the results in figure 13. It can be seen that the thermal boundary layer is slightly less influential than the viscous boundary layer and that it has most influence at the centre of the tube, where the pressure fluctuations are largest.

\section{E. Optimal positioning and sizing of a Helmholtz resonator}

Helmholtz resonators are often used in gas turbines to damp particular frequencies. They consist of a cavity, which is connected by a neck to the system that will be damped. Viscous dissipation and vortex shedding at both ends of the neck extract mechanical energy from the oscillation. The closer the oscillation is to the resonant frequency of the resonator, the larger is the oscillation in the neck, and the more acoustic oscillations are damped. In this paper, a Helmholtz resonator with mean flow through the neck is considered because this has a linear relationship between the velocity of air flowing into the resonator, $\tilde{u}_{n}$, and the pressure just outside the neck, $\tilde{p}_{n}[43, \S 5.2 .5$.$] :$

$$
\tilde{u}_{n}=\frac{\tilde{p}_{n}}{Z \bar{\rho} c_{0}} \quad \text { where } \quad Z=\frac{1-\left(\omega / \omega_{R}\right)^{2}+\mathrm{iM}_{n} \omega_{n} \omega / \omega_{R}^{2}}{\mathrm{M}_{n}+\mathrm{i} \omega_{n} \omega / \omega_{R}^{2}}
$$

where $\mathrm{M}_{n}$ is the Mach number of the mean flow in the neck, $\omega$ is the angular frequency of oscillations, $\omega_{R}$ is the resonant angular frequency of the resonator and $\omega_{n}=c_{0} /\left(L_{n}+\delta_{n}\right)$, where $L_{n}$ is the neck length and $\delta_{n}$ is the end correction. The dependence of $\omega_{R}$ and $\omega_{n}$ on the geometrical parameters of the resonator can be found in [43] and in Fig_014.m. The resonator is driven by the pressure oscillations and forces the mass equation. (In this paper, its influence on the momentum and entropy equations will be neglected for simplicity.)

For a resonator with neck cross-sectional area $S_{n}$ the fluctuating mass injection is $-\bar{\rho} S_{n} \tilde{u}_{n}$. This acts at position $X_{n}$ over a region $\delta X$. Here $\omega_{R}$ is taken to be $1020 \mathrm{rad} \mathrm{s}^{-1}$ in a chamber for which $\omega=s_{i}=1160 \mathrm{rad} \mathrm{s}^{-1}$. Following a similar analysis to that in $\S \mathrm{X} \mathrm{A}$, the dimensional eigenvalue shift is:

$$
\delta s=-\frac{P_{r e f}}{L_{r e f}} \frac{S_{n}}{S_{c}} \frac{1}{\bar{\rho} c_{0}} \frac{1}{Z}\left(\frac{\partial s}{\partial \dot{m}_{\bar{\rho}, p}}\left(X_{n}\right)\right)^{\star}=(-46.3+142 \mathrm{i})\left(\frac{\partial s}{\partial \dot{m}_{\bar{\rho}, p}}\left(X_{n}\right)\right)^{\star} .
$$


This is shown in figure 14, which is calculated with Fig_014.m. As expected, the growth rate shift depends strongly on $\left(\omega / \omega_{R}\right)$, as can be seen by changing the Helmholtz resonator geometric parameters in Fig_014.m. The main point to note, however, is that the Helmholtz resonator is orders of magnitude more influential than a cold mesh, a hot mesh, or the viscous and thermal boundary layers, as expected.

The optimal position of the Helmholtz resonator, which is the point at which the real component of $\delta s$ reaches a minimum, depends on the complex impedance of the Helmholtz resonator because $\partial s / \partial \dot{m}_{\bar{\rho}, p}$ is complex. In turn this depends on $\mathrm{M}_{n}, \omega_{n}$, and $\omega_{R}$, which depend in turn on the resonator's geometry. Further, the resonator's geometry may be constrained by the geometry of the engine. Although this leads to a technically difficult constrained optimization problem, optimization is greatly helped by the fact that the variation of $s$ with respect to every geometric parameter can be calculated with the chain rule. This can be used in a gradient-based optimization algorithm.

\section{CONCLUSIONS}

This paper shows how to implement adjoint thermoacoustic Helmholtz solvers and how to use the results for physical understanding, gradient-based optimization, and rapid estimation of the influence of retro-fitted devices and phenomena omitted from a model. The paper compares four discretization techniques (Finite Difference of the strong form with replacement boundary conditions; Finite Element of the weak form; Finite Difference of the weak form; and Summation by Parts Finite Difference of the strong form with a Simultaneous Approximation Term for the boundary conditions), two iteration techniques (a Newton method and an iterative linear method), and two adjoint techniques (discrete adjoint and continuous adjoint), making a total of 16 combinations. Matlab codes are provided to implement all 16 combinations and to create every figure in the paper.

The direct and adjoint eigenmodes are combined in different ways to reveal (i) the sensitivity of the eigenvalue to changes in the internal and external model parameters (the base state sensitivity); (ii) the sensitivity of the eigenvalue to qualitative changes to the model that introduce local feedback (the feedback sensitivity) and (iii) the receptivity of the system to open loop forcing of the mass, momentum, and energy equations. The paper shows how the receptivities and base state sensitivities aid physical understanding of the thermoa- 
coustic mechanism and how they can automate a design process to stabilize the system. The paper also shows how the feedback sensitivity can be used to test model assumptions $a$ posteriori and to identify the optimal location of retro-fitted damping devices.

Regarding numerical implications, all 16 combinations give the same result when Dirichlet boundary conditions on pressure are imposed. When Neumann or Robin boundary conditions are imposed by replacing rows in the matrix operators, the discrete adjoint of the finite difference discretization of the strong form equations (FDS_DA) contains oscillations that propagate into some of the base state and feedback sensitivities. The reasons for this are explained in $\S \mathrm{IVB}$ and $\S \mathrm{VC}$. The replacement method used for the FDS_DA method should be therefore avoided. All the other methods work well for all boundary conditions so the choice of method will depend on practical considerations, such as the methods used in any pre-existing code. If there is no pre-existing code then the easiest and safest approach is to implement the direct equations in the weak form and to use the discrete adjoint (FEW_DA, FDW_DA). This allows all discretizations to be used, with boundary conditions that are straightforward to implement. The adjoint eigenvector is then simply the left eigenvector of the corresponding generalized matrix eigenvalue problem. If the direct equations in the strong form must be used, and if the discrete adjoint is desired, then the discretization must obey a Summation by Parts formula, with boundary conditions imposed with a Simultaneous Approximation Term (SBP_DA). SBP-SAT schemes have been devised for high order schemes on uniform grids [31, 32] and on non-uniform grids [44, 45] so this is feasible, although technically more challenging than using the weak form. When using the discrete adjoint, one must not forget the relationship between the discrete and continuous sensitivities $(\S \mathrm{VD})$. The continuous adjoint can be used with any discretization but one must pay close attention to the adjoint boundary conditions $(\S \mathrm{V} \mathrm{A})$.

Expressions for the base state sensitivities are in Tab. VII and the feedback sensitivities are in Tab. VIII. These are valid in 1D, 2D, or 3D. Both iteration techniques work well but the iterative linear method ('active iteration') is more robust. If active iteration is used then the sensitivities need to be propagated through the steps as detailed in $\S \mathrm{VE}$. A neat advantage of the active iteration method is that the adjoint code can be debugged using Taylor Tests $(\S \mathrm{VF})$ with a single iteration - i.e. before the direct code has converged.

Regarding physical implications, the receptivities (§VII) give physical insight into the thermoacoustic mechanism in a given system. Comparison of the direct equation (12b) with 
the continuous adjoint equation (23b) shows that the adjoint pressure eigenfunction deviates increasingly from the pressure eigenfunction as the interaction index, $n$, increases, and as the distance between the measurement region and the heat release region increases. This is demonstrated by comparing the Rijke tube with a simple model of a rocket engine. For this reason, the quote from $\mathrm{Chu}[4]$ in $\S \mathrm{I}$ is not correct for strongly non-self-adjoint cases. Instead it is more accurate to examine the feedback sensitivities formed, correctly, with the adjoint pressure rather than the direct pressure. For the same reason, the widely-used Rayleigh criterion should be formed with the adjoint pressure rather than the pressure. The feedback sensitivities show that the viscous and thermal boundary layers can safely be omitted from the model under the conditions examined in this paper. They also show why a Helmholtz resonator damps oscillations much more than an adiabatic mesh or a hot mesh.

In summary, this paper lays out the theory, numerics, and applications of adjoint thermoacoustic Helmholtz solvers. The desirable next step is to apply these techniques to 2D and 3D Helmholtz solvers and then to apply shape optimization in order to stabilize complex geometries such as gas turbines. Engineering applications will impose further constraints, but these can be incorporated into the model or into the optimization procedure. This process has the potential to speed up the design process of gas turbines and rocket engines, whether for optimal retro-fitting of damping devices or for tweaking a current device to eliminate thermoacoustic oscillations. Given the extreme sensitivity often found in thermoacoustic systems, and hence the strong influence of small changes, adjoint Helmholtz solvers should be a valuable tool for gas turbine and rocket engine designers in the future.

\section{ACKNOWLEDGEMENTS}

The author is grateful to the Center for Turbulence Research Summer Program (Stanford University). The author wishes to thank Luca Magri for useful discussions during the course of the project and the two reviewers for their suggested improvements to the paper. 
TABLE I. Roman letters (a to m)

A operator representing acoustics and heat release without b.c.'s (eq. 12b)

A matrix representing the discretized form of A with b.c.'s (§III)

$b \quad$ generic base flow variable $(\S \mathrm{V})$

b column vector containing $b$ at the gridpoints $x_{i}$ (eq. 39)

$c_{\circ} \quad$ arbitrary constant used in the FDS replacement boundary conditions (§III A)

$c_{p} \quad$ specific heat capacity at constant pressure

C operator without b.c.'s (eq. 12c)

C matrix representing the discretized form of $\mathrm{C}$ with b.c.'s (§III)

D matrix that performs $\mathrm{d} / \mathrm{d} x(\S \mathrm{III})$

$\mathbf{e}^{\circ} \quad$ matrix containing $\mathrm{e}^{\circ}$ along the diagonal (eq. 13b)

f momentum injection per unit time (i.e. force) per unit volume (eq. 2d)

$\mathbf{F}_{\bar{\rho}, \circ}$ matrix containing $\mathbf{f}_{\bar{\rho}, \circ}$ along the diagonal $\S(\mathrm{VIC})$

$\mathrm{G}$ operator representing the governing equations $\mathrm{G} \equiv \mathrm{A}-s^{2} \mathrm{C}$ without b.c.'s (eq. 12a)

G matrix representing the discretized form of $\mathrm{G}$ with b.c.'s (§III)

$h \quad$ distribution of heat release in space $(\S I I B)$

$\mathbf{h} \quad$ column vector containing $h$ at the gridpoints $x_{i}$ (eq. $13 \mathrm{~b}$ )

$j \quad$ iteration number $(\S \mathrm{III} \mathrm{E})$

$k \quad$ Robin boundary condition $\mathrm{d} p / \mathrm{d} x=k p(\S I I I)$

$K \quad$ pressure drop coefficient $(\S \mathrm{XA})$

$l \quad$ generic local feedback mechanism (§VIB)

$L_{\circ} \quad$ length of $\circ$

L dimensions of length

$\dot{m} \quad$ mass injection per unit volume per unit time (eq. 2c)

$\dot{\mathbf{M}}_{\bar{\rho}, \circ}$ matrix containing $\dot{m}_{\bar{\rho}, \circ}$ along the diagonal (§VI C)

$\mathbf{M}$ a mass matrix such that $\langle f, g\rangle=\mathbf{f}^{H} \mathbf{M g}(\S$ III A, $\S$ IIIB)

$\overline{\mathbf{M}} \quad$ a mean matrix such that $\mathbf{x}_{0}=\overline{\mathbf{M}}_{01} \mathbf{x}_{1}(\S I I I B)$ 
TABLE II. Roman letters (n to z)

$n \quad$ interaction index in $1 \mathrm{D}$ (eq. 8b)

$N$ number of finite elements (FEW) or number of gridpoints -1 (FDS, FDW, SBP)

$p \quad$ pressure

p column vector containing $p$ at the gridpoints $x_{i}(\S \mathrm{III})$

$\mathrm{P}$ dimensions of pressure

$\dot{q} \quad$ heat release per unit volume per unit time (eq. 1e)

$\dot{\mathrm{Q}}_{\bar{p}, \circ}$ matrix containing $\dot{q}_{\bar{p}, \circ}$ along the diagonal (§VIC)

$R$ reflection coefficient $(\S I I I \mathrm{~A})$

$r_{g} \quad$ specific gas constant (eq. 1e)

$S_{\circ} \quad$ cross-sectional area of $\circ(\S I I D)$

$s \quad$ complex angular frequency $(\S \mathrm{II} \mathrm{E})$

S matrix containing boundary terms for the SBP-SAT formulation ( $\S$ III D)

$T$ temperature (eq. 1a)

$t \quad$ time

$\underline{\mathbf{u}} \quad$ velocity vector in $3 \mathrm{D}$ (eq. 1c)

$u \quad$ velocity in $x$-direction $(\S \mathrm{IID})$

$\mathbf{u} \quad$ column vector containing values of $u$ at the gridpoints (§III)

$\mathrm{U}$ dimensions of velocity

$v \quad$ specific volume, $v \equiv \rho^{-1}(\S I \mathrm{C}$ )

$\mathbf{v} \quad$ column vector containing $v\left(x_{i}\right)$

V matrix containing $\bar{v}\left(x_{i}\right)$ along the diagonal (§III)

$\tilde{\mathbf{V}} \quad \operatorname{matrix} \operatorname{diag}\left(-v_{d}, 0, \ldots, 0, v_{u}\right)(\S \mathrm{IIID})$

$w \quad$ distribution of the measurement region in space (eq. 4)

w column vector containing the values of $w$ at the gridpoints $x_{i}(\S \mathrm{III})$

$\underline{\hat{\mathbf{w}}}$ unit vector in the measurement direction (eq. 4)

$\underline{\mathbf{x}}$ coordinates in $3 \mathrm{D}$

$x \quad$ coordinate in $1 \mathrm{D}(\S \mathrm{IID})$

$\mathbf{x} \quad$ column vector containing the values of $x$ at the gridpoints (§III)

$X \circ$ Location of 。

$z \quad$ test function (eq. 14)

$\mathbf{z} \quad$ column vector containing $z$ at thqpridpoints $x_{i}$ (§III B $\S$ III C)

$Z \quad$ complex impedance (eq. 56) 
TABLE III. Greek letters

$\alpha$ normalization inner product (denominator of eq. 33b (CA) or eq. 38 (DA))

$\gamma$ ratio of specific heat capacities, $c_{p} / c_{v}(\S \mathrm{II} \mathrm{A})$

$\Gamma_{c}$ perimeter of the chamber (Fig 8)

$\delta$ a change at order $\epsilon(\S I I \mathrm{~A})$

$\delta_{b l}$ acoustic boundary layer thickness $(\S \mathrm{X} \mathrm{C}$ and $\S \mathrm{XD})$

$\Delta$ number of spatial dimensions being considered: 1,2 or 3 (§V D)

$\epsilon \quad$ a small number $(\S I I \mathrm{~A})$

$\zeta$ thermal expansion factor $(\gamma-1) / \gamma(\S I I \mathrm{C})$

$\eta$ interaction index in 3D (eq. 4)

$\lambda$ thermal conductivity of gas $(\S \mathrm{X} \mathrm{D})$

$\nu$ kinematic viscosity

$\xi_{j} \partial s_{j} / \partial s_{j-1}$ defined in (eq. 42$)$

$\rho$ density (eq. 1c)

$\varsigma \quad$ specific entropy (eq. 1b)

$\tau$ time delay between $\underline{\tilde{\mathbf{u}}}$ measured at $w(\underline{\mathbf{x}})$ and $\tilde{\dot{q}}$ experienced at $h(\underline{\mathbf{x}})(\S \mathrm{II} \mathrm{B})$

$\chi_{j} \prod_{J-j}^{J} \xi_{j}($ eq. 43$)$

$\omega$ angular frequency (real)

$\Omega$ the $3 \mathrm{D}$ domain (eq. 4) 


\section{TABLE IV. Subscripts}

$\circ_{0} \quad$ a $\mathrm{P} 0$ function, piecewise constant between gridpoints at $\mathbf{x}_{1}(\S I I I \mathrm{~B})$

$\circ_{1} \quad$ a P1 function, piecewise linear between gridpoints at $\mathbf{x}_{1}$ (§III B)

$\bigcirc_{00}$ a matrix that maps a $\mathrm{P} 0$ function to a $\mathrm{P} 0$ function (§III B)

$\bigcirc_{01}$ a matrix that maps a $\mathrm{P} 1$ function to a P0 function (§III B)

${ }^{\circ} 11$ a matrix that maps a $\mathrm{P} 1$ function to a $\mathrm{P} 1$ function (§III B)

$\circ_{a} \quad$ value of $\circ$ at ambient conditions ( $(I I C)$

$\circ_{c} \quad \circ$ of the chamber $(\S I I D)$

$\circ_{d} \quad$ value of $\circ$ at the downstream boundary (§III A)

$\circ_{f} \quad$ value of $\circ$ at the forcing frequency $(\S \mathrm{VII})$

$\circ_{h} \quad$ value of $\circ$ at the point of heat release (eq. 1e)

$\circ_{i} \quad$ value of $\circ$ at the gridpoint $i(\S$ III A $\S$ III B)

$\circ_{j} \quad$ value of $\circ$ at the iteration $j(\S \mathrm{IIIE}$ )

$\circ_{J} \quad$ value of $\circ$ at the final iteration, $J(\S \mathrm{III} \mathrm{E})$

$\circ_{k} \quad$ the $k^{t h}$ eigenmode of $\circ(\S \mathrm{VII})$

$\circ_{m} \quad$ value of $\circ$ at the control mesh $(\S \mathrm{XA} \S \mathrm{XB})$

$\circ_{n} \quad$ value of $\circ$ at the Helmholtz resonator neck ( $\left.\S \mathrm{X} \mathrm{E}\right)$

$\circ_{\bar{p}} \quad \circ / \bar{p}($ eq. 6$)$

$\circ_{\bar{p}, \star}$ contribution to $\circ_{\bar{p}}$ from $\star$ (eq. 46)

$\circ \bar{\rho} \quad \circ / \bar{\rho}($ eq. 6$)$

$\bigcirc_{\bar{\rho}, \star}$ contribution to $\odot_{\bar{\rho}}$ from $\star$ (eq. 46)

$\circ_{\text {ref }}$ reference value of o $(\S \mathrm{II} \mathrm{C})$

$\circ_{u} \quad$ value of $\circ$ at the upstream boundary (§III A)

$\circ_{w} \quad$ value of $\circ$ at the point of measurement (§II B) 
TABLE V. Superscripts, Hats and underlines, Brackets, and Labels

$\circ^{*} \quad$ complex conjugate of $\circ$

॰ non-dimensional ○ $(\S \mathrm{II} \mathrm{C})$

o $\quad$ adjoint of $\circ(\S \mathrm{IV})$

$\circ^{T} \quad$ transpose of $\circ$

$\circ^{H} \quad$ conjugate transpose of $\circ$

$\circ^{\prime} \quad$ first derivative of $\circ$

$\hat{o}(\underline{\mathbf{x}}, t)$ total value $($ mean + perturbation $)(\S I I A)$

$\overline{\mathrm{o}}(\underline{\mathbf{x}}) \quad$ mean value $(\S \mathrm{II} \mathrm{A})$

$\tilde{o}(\underline{\mathbf{x}}, t)$ perturbation in the time domain (§II A)

$\circ(\underline{\mathbf{x}}, s)$ perturbation in the frequency domain (§II E)

○ dummy variable

은 vector

$\langle f, g\rangle \quad$ inner product $\int_{0}^{1} f^{*} g \mathrm{~d} x$ (eq. 11)

$[\circ]_{d}^{u} \quad$ boundary terms: $\circ_{u}-\circ_{d}$ (eq. 14)

Pr Prandtl number

M Mach number

FDS finite difference of the strong form equations with replacement boundary conditions (§III A)

FEW finite element of the weak form (§III B)

FDW finite difference of the weak form equations (§III C)

SBP finite difference of the strong form equations in SAT-SBP form (§IIID)

CA continuous adjoint (eq. 20)

DA discrete adjoint (eq. 22)

nonlin Newton method applied to a nonlinear eigenvalue problem

linear 'active iteration' method; a sequence of linear eigenvalue problems [1] 
TABLE VI. Dimensional quantities, distributions of density, $\bar{\rho}(x)$, heat release region, $h(x)$, and measurement region $w_{\bar{\rho}}$, and non-dimensional quantities for (left) the Rijke tube in [33] and (right) a simplified model of a $\mathrm{H}_{2} / \mathrm{LOx}$ rocket engine.

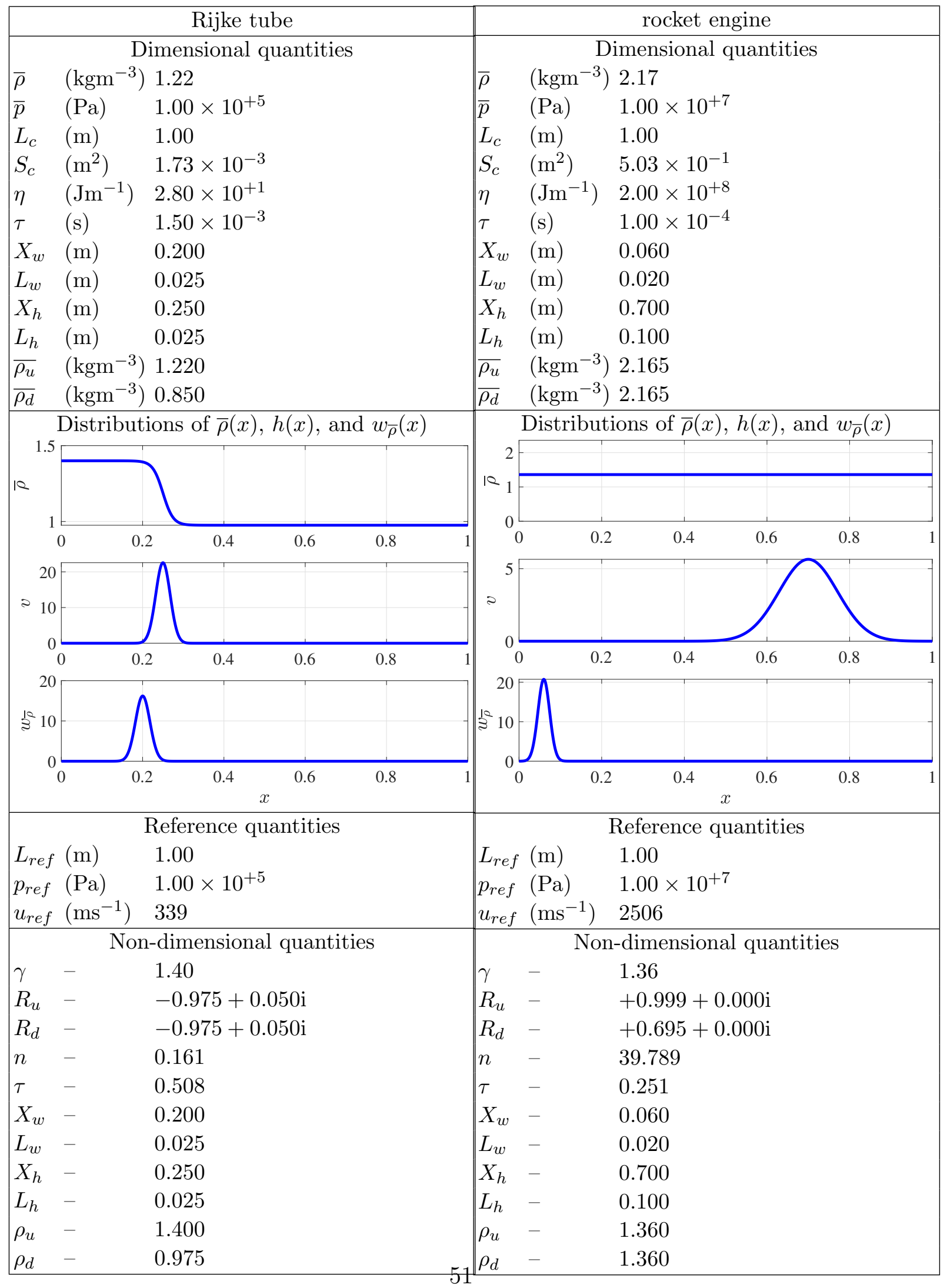



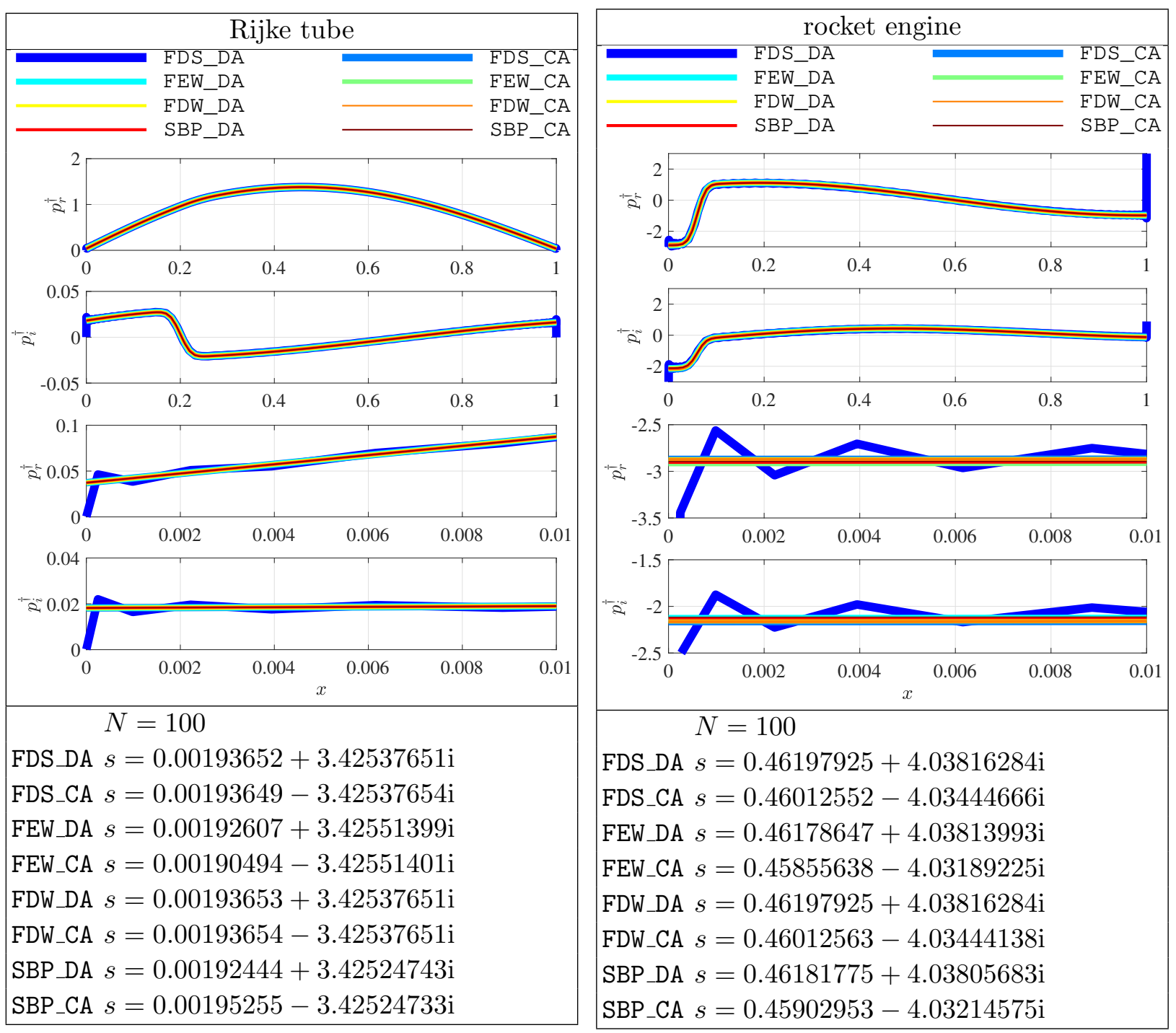

FIG. 2. (Color online) Adjoint pressure, $p^{\dagger}$, eigenfunctions calculated with four different spatial discretizations (FDS, FEW, FDW, SBP) either with the discrete adjoint method DA or the continuous adjoint method CA. The bottom two frames show a zoom around the boundaries, revealing oscillations in the discrete adjoint of the finite difference of the strong form equations with replacement boundary conditions (FDS_DA). For the rocket engine, the CA and DA eigenfunctions and eigenvalues differ slightly from each other because $w_{\bar{\rho}}$ is not quite zero at the boundary (order $10^{-3}$ ) so the neglected term in (24) has a slight influence. This figure is created with Fig_002.m. By running this with larger $N$, one can check that the eigenvalues are the same to the requested tolerance. 
TABLE VII. Base state sensitivities derived in the continuous framework (33), discretized strong form, discretized weak form, and SBP-SAT discretized strong form. The inner product $\alpha$ is defined in (33b) and(38). Expressions within curly brackets $\{\circ\}$ and the inner product $\langle\circ, \circ\rangle$ are numbers. Expressions within braces (o) are functions of $x$ in the continuous framework and row vectors in the discrete frameworks. The symbol $\odot$ denotes element by element multiplication of two row vectors.

\begin{tabular}{|c|c|c|}
\hline & Continuous (*_CA) & Discretized strong form (FDS_DA) \\
\hline$\left\{\partial s / \partial k_{u}\right\}$ & $p_{u}^{\dagger *} \bar{v}_{u} p_{u} / \alpha$ & $\mathbf{p}_{u}^{\dagger *} c_{u} \mathbf{p}_{u} / \alpha$ \\
\hline$\left\{\partial s / \partial k_{d}\right\}$ & $p_{d}^{\dagger *} \bar{v}_{d} p_{d} / \alpha$ & $-\mathbf{p}_{d}^{\dagger *} c_{d} \mathbf{p}_{d} / \alpha$ \\
\hline$(\partial s / \partial \bar{v})$ & $-\left(\frac{\mathrm{d} p^{\dagger}}{\mathrm{d} x}\right)^{*}\left(\frac{\mathrm{d} p}{\mathrm{~d} x}\right) / \alpha$ & $\left(\mathbf{p}^{\dagger^{H}} \mathbf{M D}\right) \odot(\mathbf{D} \mathbf{p})^{T} / \alpha$ \\
\hline$\{\partial s / \partial n\}$ & $-\left\langle p^{\dagger^{*}}, \zeta\left(\mathrm{e}^{-s \tau}\right) h\right\rangle\left\langle w_{\bar{\rho}}^{*}, \frac{\mathrm{d} p}{\mathrm{~d} x}\right\rangle / \alpha$ & $-\left\{\mathbf{p}^{\dagger H} \mathbf{M} \zeta\left(\mathbf{e}^{-s \tau}\right) \mathbf{h}\right\} \times\left\{\mathbf{w} \frac{T}{\rho} \mathbf{M D p}\right\} / \alpha$ \\
\hline$(\partial s / \partial \tau)$ & $\left(p^{\dagger^{*}}\right)\left(\zeta n s\left(\mathrm{e}^{-s \tau}\right) h\right)\left\langle w_{\bar{\rho}}^{*}, \frac{\mathrm{d} p}{\mathrm{~d} x}\right\rangle / \alpha$ & $\left(\mathbf{p}^{\dagger^{H}} \mathbf{M}\right) \odot\left(\zeta n s\left(\mathbf{e}^{-s \tau}\right) \mathbf{h}\right)^{T} \times\left\{\mathbf{w}_{\bar{\rho}}^{T} \mathbf{M D p}\right\} / \alpha$ \\
\hline$(\partial s / \partial h)$ & $-\left(p^{\dagger^{*}}\right)\left(\zeta n\left(\mathrm{e}^{-s \tau}\right)\right)\left\langle w_{\bar{\rho}}^{*}, \frac{\mathrm{d} p}{\mathrm{~d} x}\right\rangle / \alpha$ & $-\left(\mathbf{p}^{\dagger}{ }^{H} \mathbf{M} \zeta n\left(\mathbf{e}^{-s \tau}\right)\right) \times\left\{\mathbf{w} \frac{T}{\rho} \mathbf{M D} \mathbf{p}\right\} / \alpha$ \\
\hline \multirow[t]{2}{*}{$\left(\partial s / \partial w_{\bar{\rho}}^{*}\right)$} & $-\left\langle p^{\dagger^{*}}, \zeta n\left(\mathrm{e}^{-s \tau}\right) h\right\rangle\left(\frac{\mathrm{d} p}{\mathrm{~d} x}\right) / \alpha$ & $-\left\{\mathbf{p}^{\dagger^{H}} \mathbf{M} \zeta n\left(\mathbf{e}^{-s \tau}\right) \mathbf{h}\right\} \times(\mathbf{M D} \mathbf{p})^{T} / \alpha$ \\
\hline & Discretized weak form (FEW_DA, FDW_DA) & SBP-SAT (SBP_DA) \\
\hline$\left\{\partial s / \partial k_{u}\right\}$ & $\mathbf{p}_{u}^{\dagger_{u}^{*}} \bar{v}_{u} \mathbf{p}_{u} / \alpha$ & $\mathbf{p}^{\dagger_{u}^{*}} \bar{v}_{u} \mathbf{p}_{u} / \alpha$ \\
\hline$\left\{\partial s / \partial k_{d}\right\}$ & $\mathbf{p}_{d}^{\dagger^{*} \bar{v}_{d} \mathbf{p}_{d} / \alpha}$ & $\mathbf{p}_{d}^{\dagger^{*} \bar{v}_{d} \mathbf{p}_{d} / \alpha}$ \\
\hline$(\partial s / \partial \bar{v})$ & $-\left(\mathbf{p}_{1}^{\dagger}{ }_{1}^{H} \mathbf{D}_{01}^{H} \mathbf{M}_{00}\right) \odot\left(\mathbf{D}_{01} \mathbf{p}_{1}\right)^{T} / \alpha$ & $-\left(\mathbf{p}^{\dagger}{ }^{H} \mathbf{D}_{01}^{H} \mathbf{M}_{00}\right) \odot\left(\mathbf{D}_{01} \mathbf{p}\right)^{T} / \alpha$ \\
\hline$\{\partial s / \partial n\}$ & $-\left\{\mathbf{p}_{1}^{\dagger H} \mathbf{M}_{11} \zeta\left(\mathbf{e}^{-s \tau_{1}}\right) \mathbf{h}_{1}\right\} \times\left\{\mathbf{w}_{\bar{\rho} 0}^{T} \mathbf{M}_{00} \mathbf{D}_{01} \mathbf{p}\right\} / \alpha$ & $-\left\{\mathbf{p}^{\dagger H} \mathbf{M} \zeta\left(\mathbf{e}^{-s \tau}\right) \mathbf{h}\right\} \times\left\{\mathbf{w}_{\bar{\rho}}^{T} \mathbf{M D}_{1} \mathbf{p}\right\} / \alpha$ \\
\hline$(\partial s / \partial \tau)$ & $\left(\mathbf{p}_{1}^{\dagger H} \mathbf{M}_{11}\right) \odot\left(\zeta n s\left(\mathbf{e}^{-s \tau_{1}}\right) \mathbf{h}_{1}\right)^{T} \times\left\{\mathbf{w}_{\bar{\rho} 0}^{T} \mathbf{M}_{00} \mathbf{D}_{01} \mathbf{p}_{1}\right\} / \alpha$ & $\left(\mathbf{p}^{\dagger H} \mathbf{M}\right) \odot\left(\zeta n s\left(\mathbf{e}^{-s \tau}\right) \mathbf{h}\right)^{T} \times\left\{\mathbf{w}_{\frac{T}{\rho}} \mathbf{M} \mathbf{D}_{1} \mathbf{p}\right\} / \alpha$ \\
\hline$(\partial s / \partial h)$ & $-\left(\mathbf{p}_{1}^{\dagger}{ }_{1}^{H} \mathbf{M}_{11} \zeta n\left(\mathbf{e}^{-s \tau_{1}}\right)\right) \times\left\{\mathbf{w}_{\bar{\rho} 0}^{T} \mathbf{M}_{00} \mathbf{D}_{01} \mathbf{p}_{1}\right\} / \alpha$ & $-\left(\mathbf{p}^{\dagger^{H}} \mathbf{M} \zeta n\left(\mathbf{e}^{-s \tau}\right)\right) \times\left\{\mathbf{w}_{\bar{\rho}}^{T} \mathbf{M} \mathbf{D}_{1} \mathbf{p}\right\} / \alpha$ \\
\hline$\left(\partial s / \partial w_{\bar{\rho}}^{*}\right)$ & $-\left\{\mathbf{p}_{1}^{\dagger}{ }_{1}^{H} \mathbf{M}_{11} \zeta n\left(\mathbf{e}^{-s \tau_{1}}\right) \mathbf{h}_{1}\right\} \times\left(\mathbf{M}_{00} \mathbf{D}_{01} \mathbf{p}_{1}\right)^{T} / \alpha$ & $-\left\{\mathbf{p}^{\dagger H} \mathbf{M} \zeta n\left(\mathbf{e}^{-s \tau}\right) \mathbf{h}\right\} \times\left(\mathbf{M D}_{1} \mathbf{p}\right)^{T} / \alpha$ \\
\hline
\end{tabular}



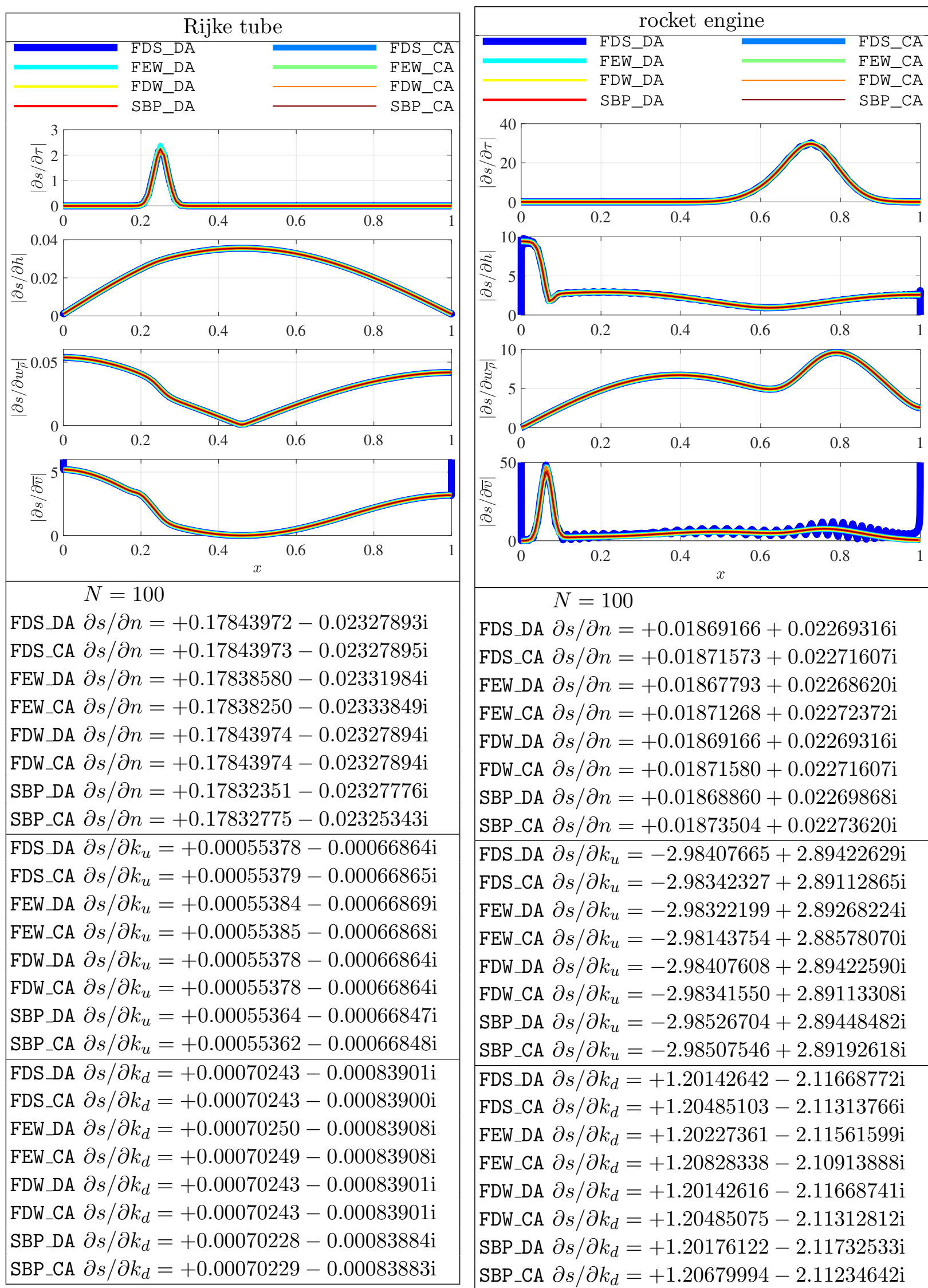

$N=100$

FDS_DA $\partial s / \partial n=+0.01869166+0.02269316 \mathrm{i}$ FDS_CA $\partial s / \partial n=+0.01871573+0.02271607 \mathrm{i}$ FEW_DA $\partial s / \partial n=+0.01867793+0.02268620 \mathrm{i}$ FEW_CA $\partial s / \partial n=+0.01871268+0.02272372 \mathrm{i}$ FDW_DA $\partial s / \partial n=+0.01869166+0.02269316 \mathrm{i}$ FDW_CA $\partial s / \partial n=+0.01871580+0.02271607 \mathrm{i}$ SBP_DA $\partial s / \partial n=+0.01868860+0.02269868 \mathrm{i}$ SBP_CA $\partial s / \partial n=+0.01873504+0.02273620 \mathrm{i}$ FDS_DA $\partial s / \partial k_{u}=-2.98407665+2.89422629 \mathrm{i}$ FDS_CA $\partial s / \partial k_{u}=-2.98342327+2.89112865 \mathrm{i}$ FEW_DA $\partial s / \partial k_{u}=-2.98322199+2.89268224 \mathrm{i}$ FEW_CA $\partial s / \partial k_{u}=-2.98143754+2.88578070 \mathrm{i}$ FDW_DA $\partial s / \partial k_{u}=-2.98407608+2.89422590 \mathrm{i}$ FDW_CA $\partial s / \partial k_{u}=-2.98341550+2.89113308 \mathrm{i}$ SBP_DA $\partial s / \partial k_{u}=-2.98526704+2.89448482 \mathrm{i}$ SBP_CA $\partial s / \partial k_{u}=-2.98507546+2.89192618 \mathrm{i}$ FDS_DA $\partial s / \partial k_{d}=+1.20142642-2.11668772 \mathrm{i}$ FDS_CA $\partial s / \partial k_{d}=+1.20485103-2.11313766 \mathrm{i}$ FEW_DA $\partial s / \partial k_{d}=+1.20227361-2.11561599 \mathrm{i}$ FEW_CA $\partial s / \partial k_{d}=+1.20828338-2.10913888 \mathrm{i}$ FDW_DA $\partial s / \partial k_{d}=+1.20142616-2.11668741 \mathrm{i}$ FDW_CA $\partial s / \partial k_{d}=+1.20485075-2.11312812 \mathrm{i}$ SBP_DA $\partial s / \partial k_{d}=+1.20176122-2.11732533 \mathrm{i}$ SBP_CA $\partial s / \partial k_{d}=+1.20679994-2.11234642 \mathrm{i}$

FIG. 3. (Color online) Absolute values of the base state sensitivities $\left(\partial s / \partial \tau, \partial s / \partial h, \partial s / \partial w_{\bar{\rho}}\right.$, $\left.\partial s / \partial \bar{v}, \partial s / \partial n, \partial s / \partial k_{u}, \partial s / \partial k_{d}\right)$ calculated with 54 the discrete adjoint method (DA) and continuous adjoint method (CA) for four spatial discretizations (FDS, FEW, FDW, SBP). All lines except FDS_DA lie 


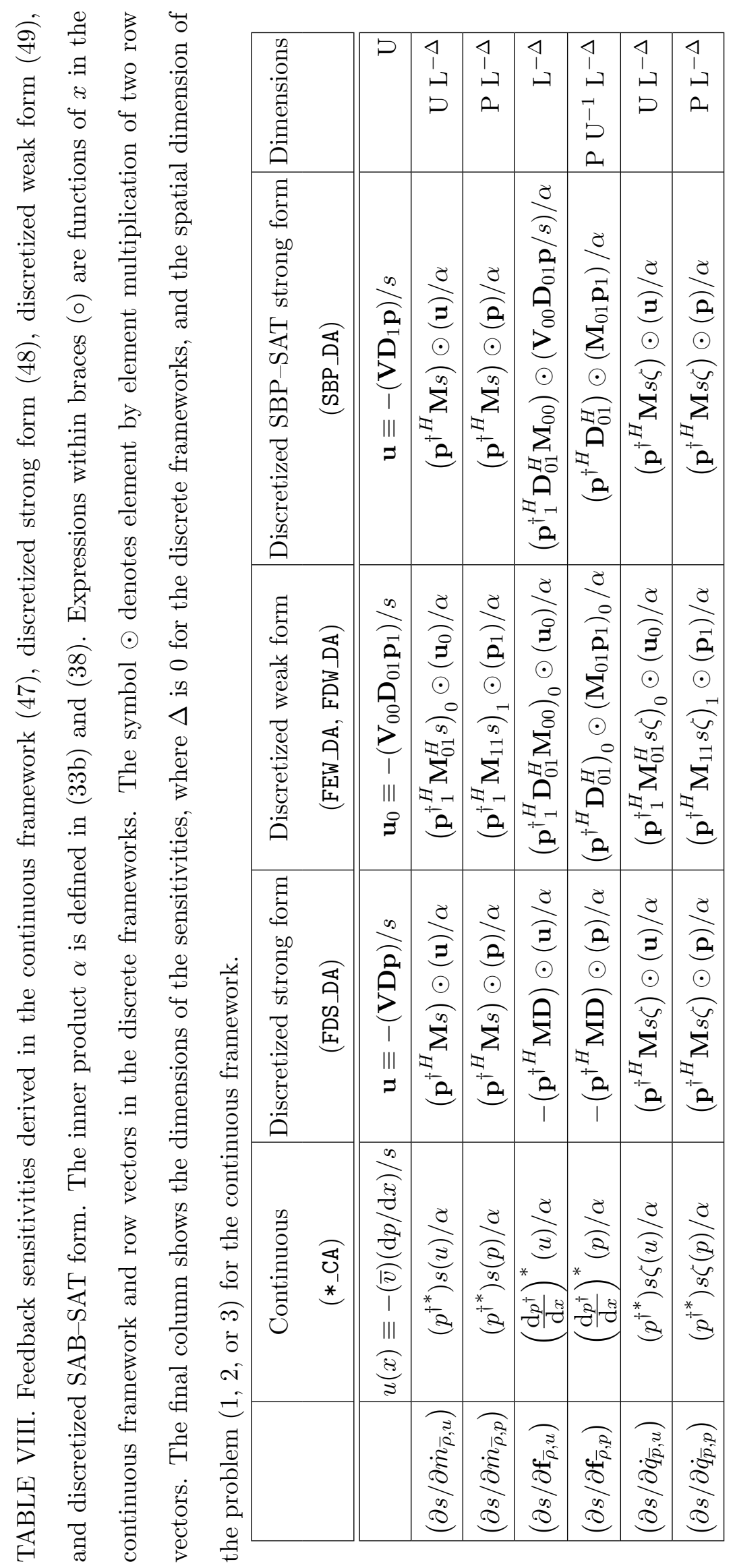



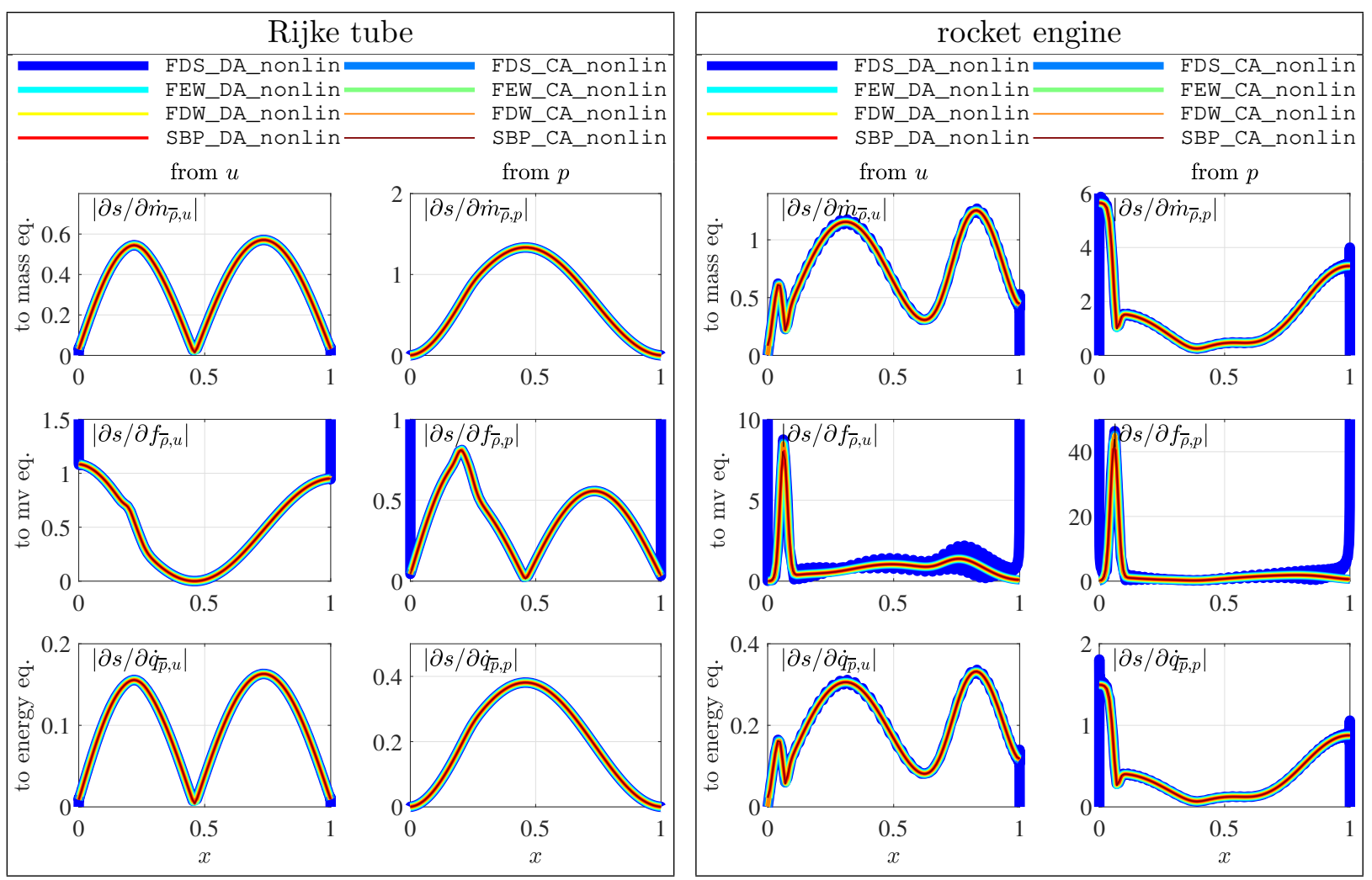

FIG. 4. (Color online) Absolute values of the feedback sensitivities $\left(\partial s / \partial \dot{m}_{\bar{\rho}, u}, \partial s / \partial \dot{m}_{\bar{\rho}, p}, \partial s / \partial \mathbf{f}_{\bar{\rho}, u}\right.$, $\left.\partial s / \partial \mathbf{f}_{\bar{\rho}, p}, \partial s / \partial \dot{q}_{\bar{p}, u}, \partial s / \partial \dot{q}_{\bar{p}, p}\right)$ calculated with the discrete adjoint method (DA) and continuous adjoint method (CA) for four spatial discretizations (FDS, FEW, FDW, SBP). All lines lie on top of each other except FDS_DA, which oscillates. These results were created with Fig_004.m. 

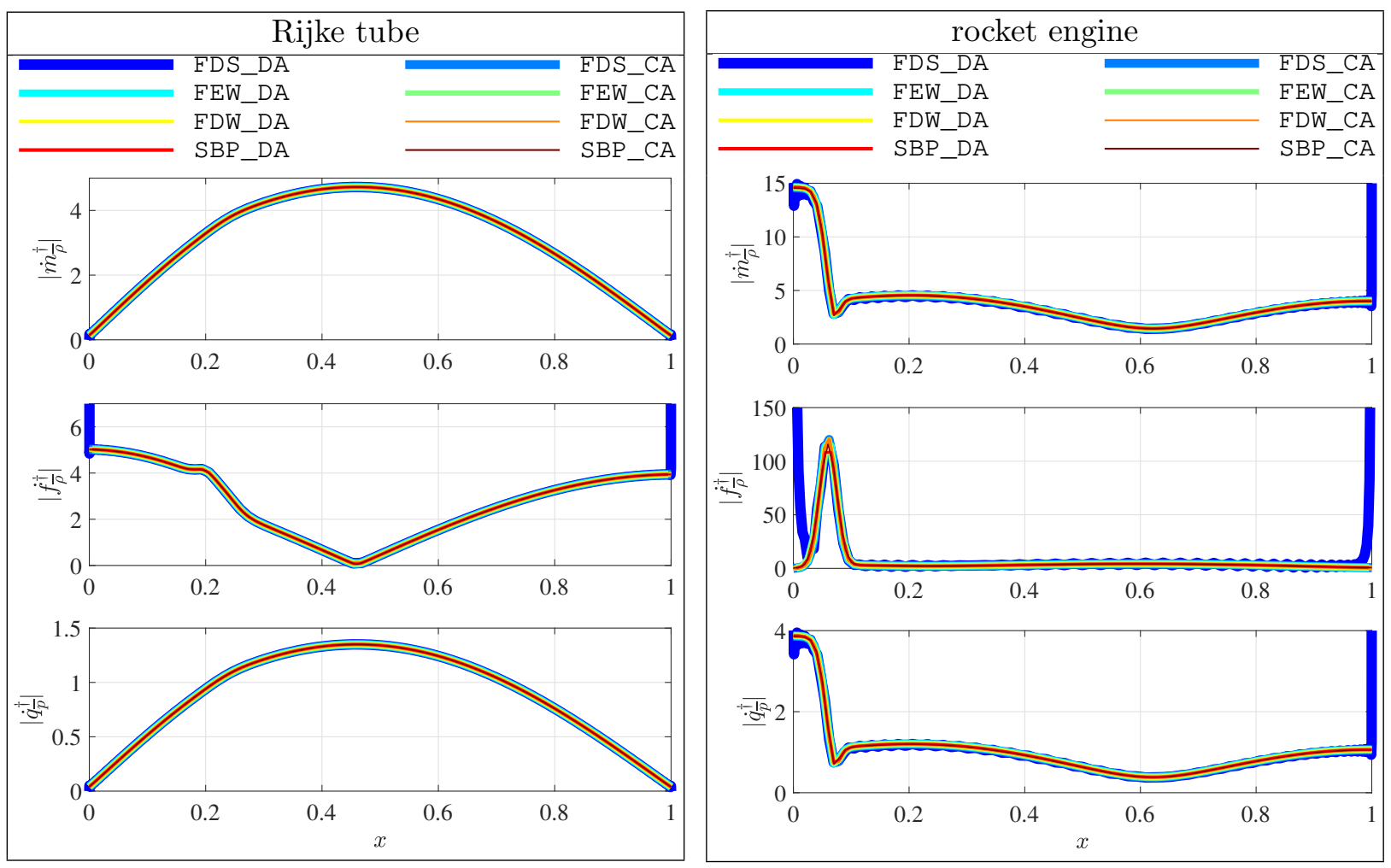

FIG. 5. (Color online) Absolute values of the receptivity of the eigenvalue to periodic injection of $\dot{m}_{\bar{\rho}}(x) \mathrm{e}^{s_{f} t}$ into the mass equation $(2 \mathrm{c}), \mathbf{f}_{\bar{\rho}}(x) \mathrm{e}^{s_{f} t}$ into the momentum equation $(2 \mathrm{~d})$, and $\dot{q}_{\bar{p}}(x) \mathrm{e}^{s_{f} t}$ into the energy equation (2e), where $s_{f}$ is an imaginary number equal to the angular frequency of forcing. From (6), the receptivity of the eigenvalue to mass injection into the mass equation is $\dot{m}_{\bar{\rho}}^{\dagger} / \bar{\rho}$, momentum injection into the momentum equation is $\mathbf{f}_{\bar{\rho}}^{\dagger} / \bar{\rho}$, and heat injection into the energy equation is $\dot{q}_{\bar{p}}^{\dagger} / \bar{p}$. These are calculated with the discrete adjoint method (DA) and continuous adjoint method (CA) for four spatial discretizations (FDS, FEW, FDW, SBP). All lines lie on top of each other except FDS_DA, which oscillates. This figure is created with Fig_005.m 

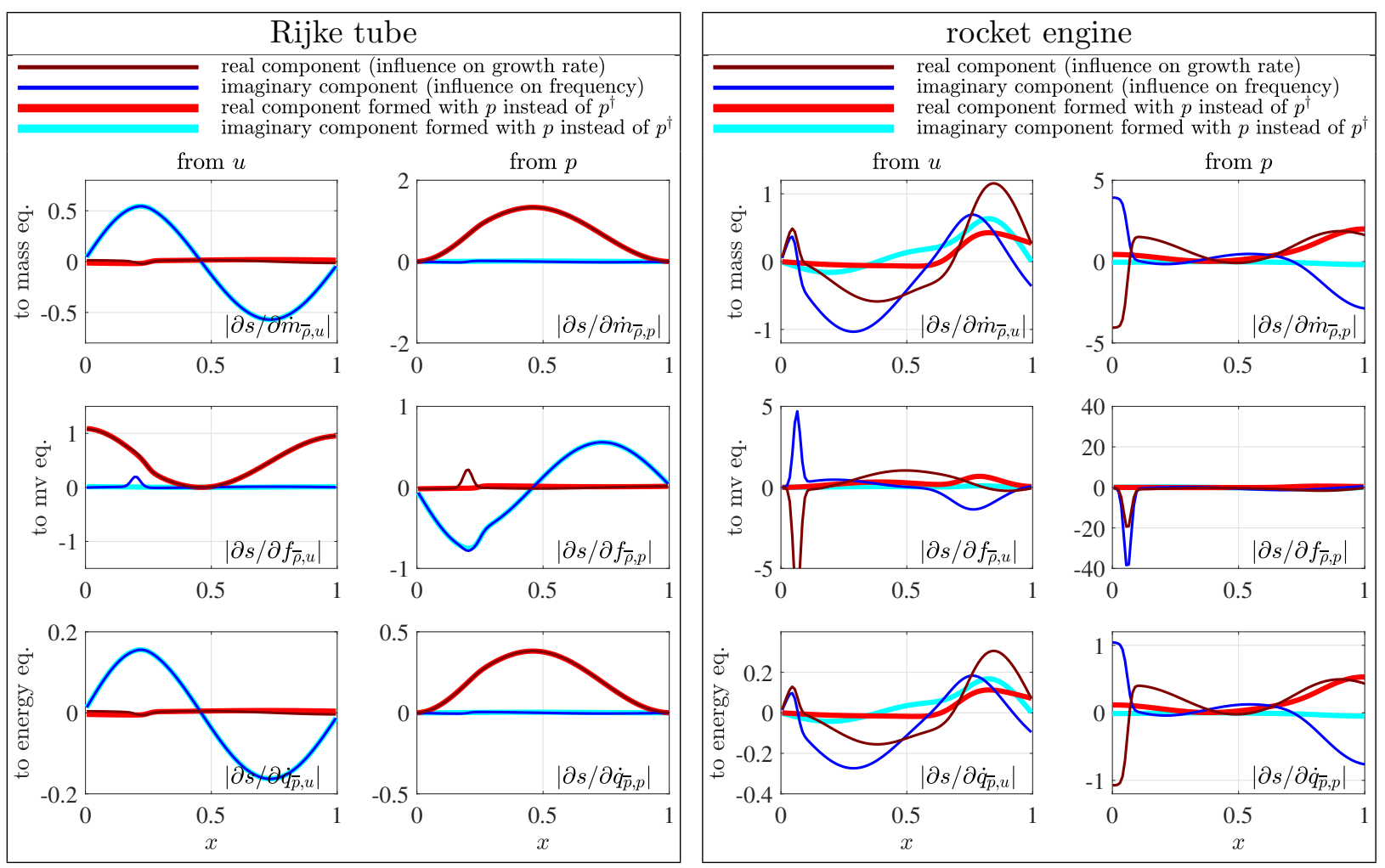

FIG. 6. (Color online) Real and imaginary components of the feedback sensitivities in figure 4, calculated with discrete adjoint (DA) of the finite element method (FEW). The wider lines show the same sensitivities calculated when the adjoint pressure, $p^{\dagger}$, is replaced by the direction pressure, $p$, i.e. as if the system were self-adjoint. There is little difference for the Rijke tube, which is weakly non-self-adjoint but a large difference for the rocket engine, which is strongly non-self-adjoint. This figure is created with Fig_006.m. 

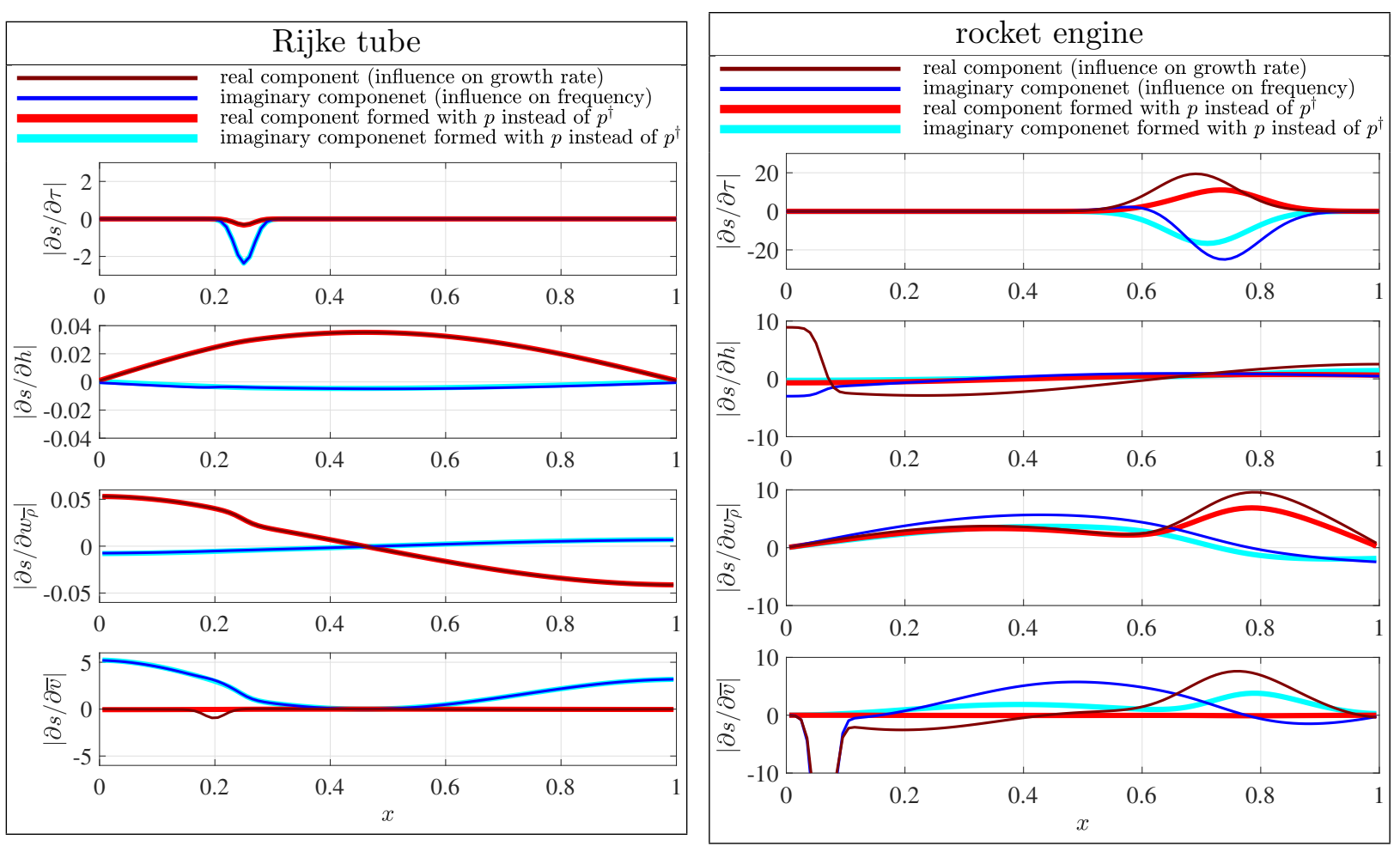

FIG. 7. (Color online) Real and imaginary components of the base state sensitivities in figure 3, calculated with discrete adjoint (DA) of the finite element method (FEW). As in Fig. 6, the wider lines show the base state sensitivities formed when the adjoint pressure, $p^{\dagger}$, is replaced by the direction pressure, $p$, i.e. if the system is assumed to be self-adjoint. This figure is created with Fig_007.m 


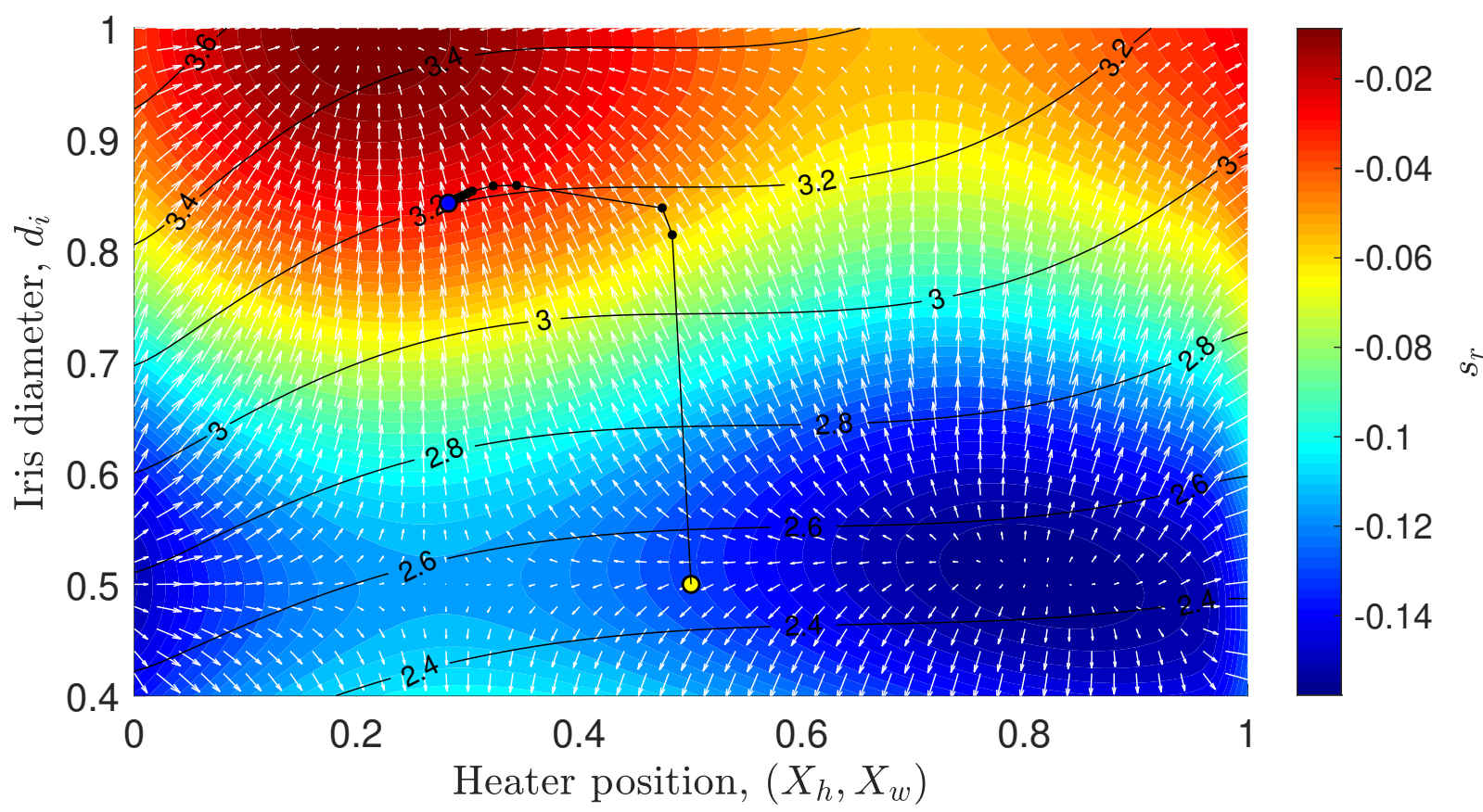

FIG. 8. (Color online) Growth rates (color contours) and non-dimensional frequencies (black line contours) for the Rijke tube with a variable-diameter iris placed at the downstream boundary and a variable heater position, $\left(X_{h}, X_{w}\right)$. A quasi-Newton algorithm uses adjoint-based gradient information (white arrows) to converge to the point with maximum growth rate at a user-defined frequency, in this case 3.2. This figure is created with Fig_008.m

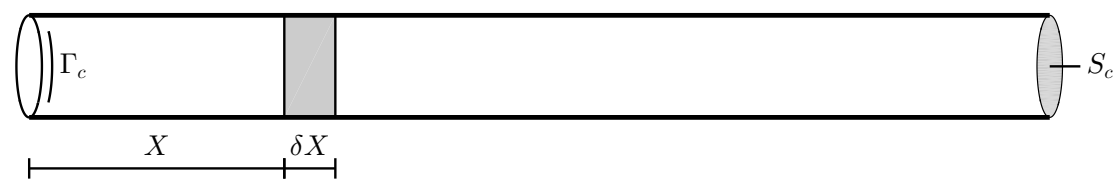

FIG. 9. Diagram of a Rijke tube with perimeter $\Gamma_{c}$ and cross-sectional area $S_{c}$ containing a passive feedback device at position $X$ with width $\delta X$. 


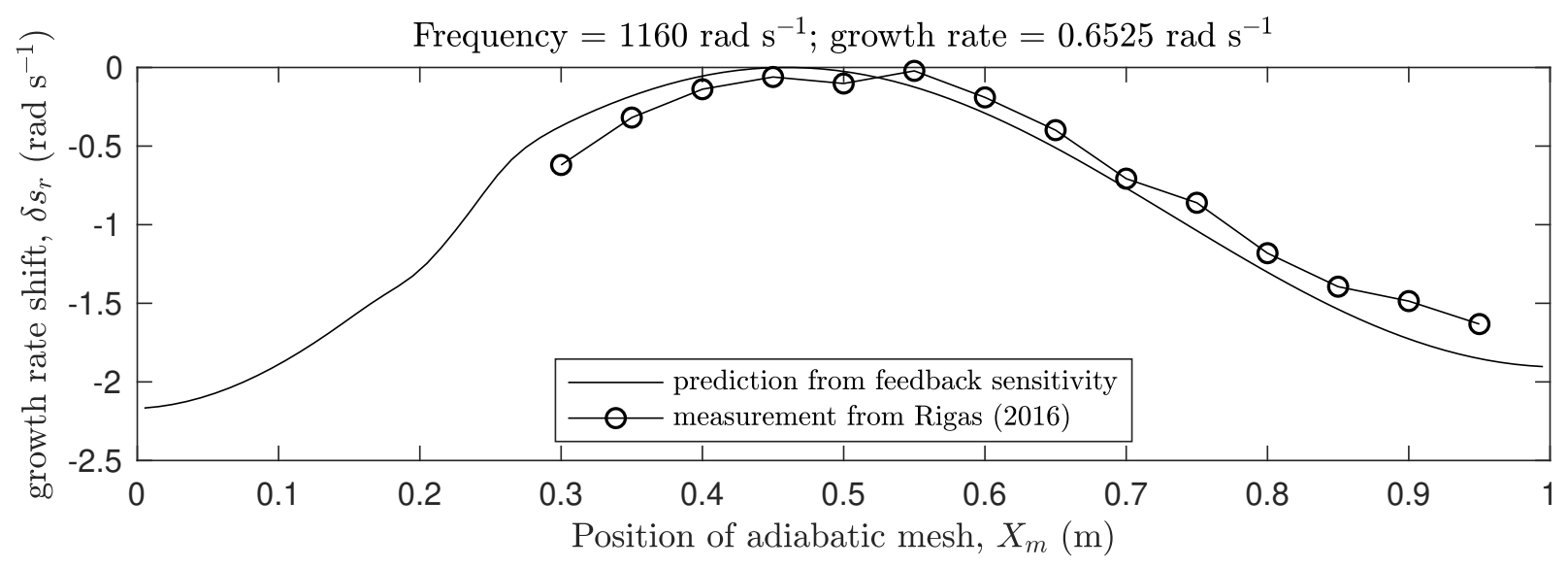

FIG. 10. Growth rate shift caused by drag from an adiabatic mesh placed at position $X_{m}$.

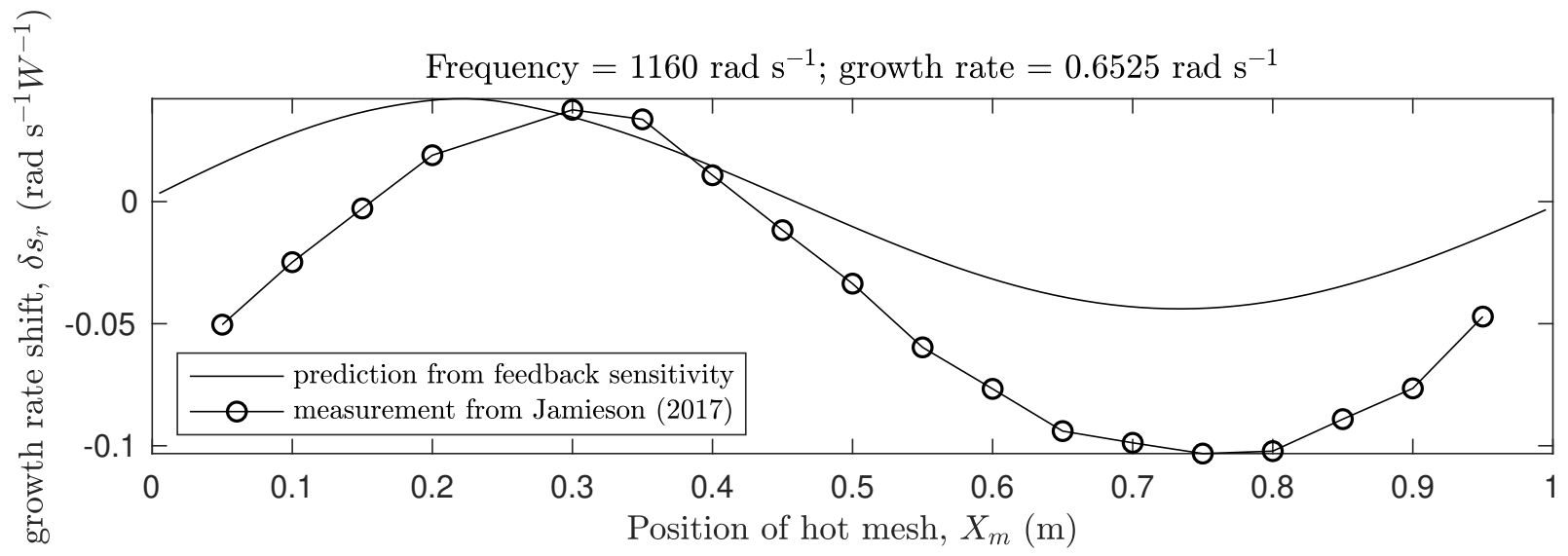

FIG. 11. Growth rate shift caused by drag from a secondary hot mesh placed at position $X_{m}$. The off-set is caused by a change to the base state induced by the secondary hot mesh [42]. 


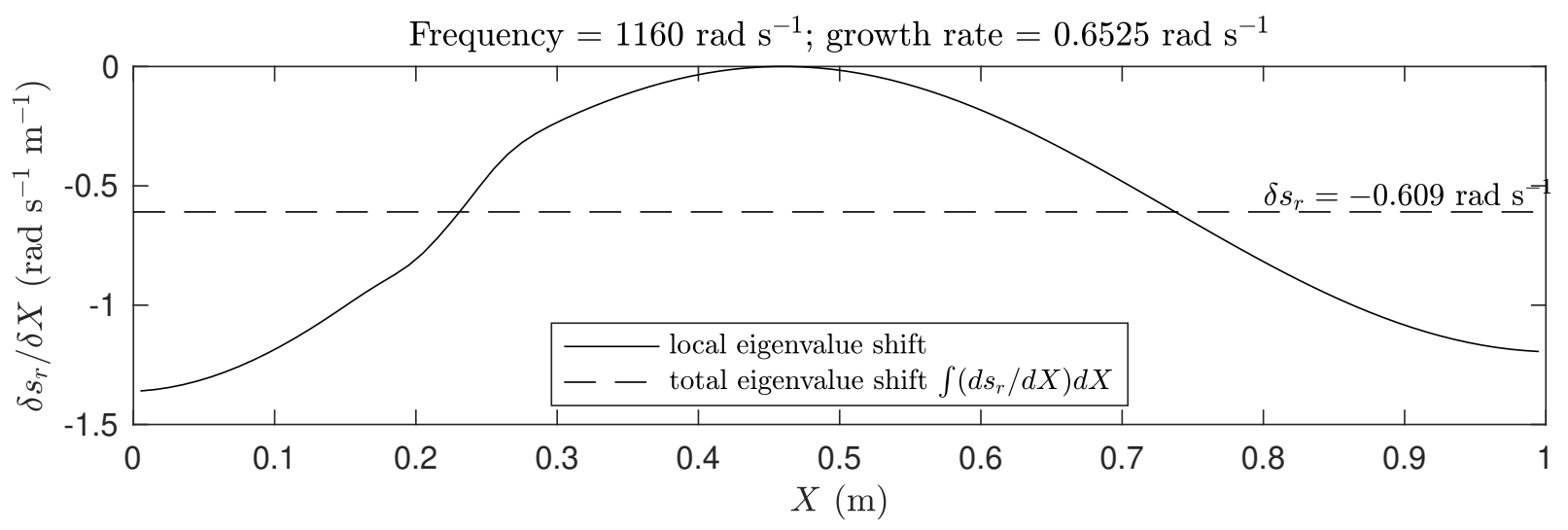

FIG. 12. Growth rate shift caused by the viscous acoustic boundary layer, estimated with the feedback sensitivity.

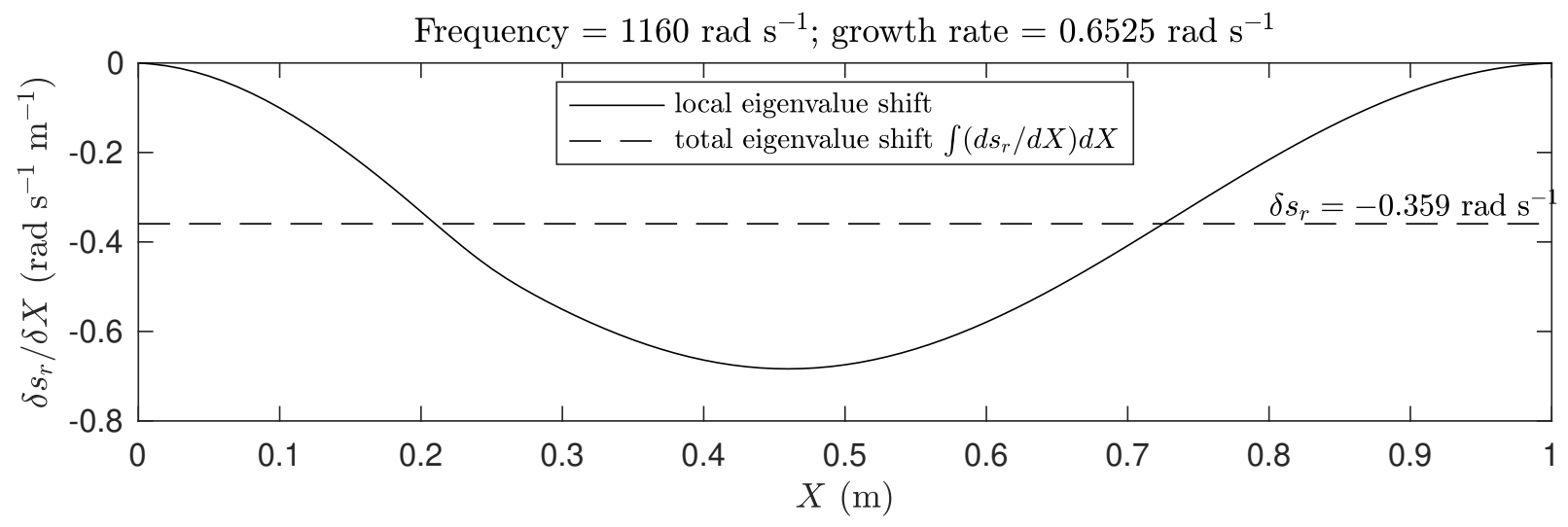

FIG. 13. Growth rate shift caused by the thermal acoustic boundary layer, estimated with the feedback sensitivity. 


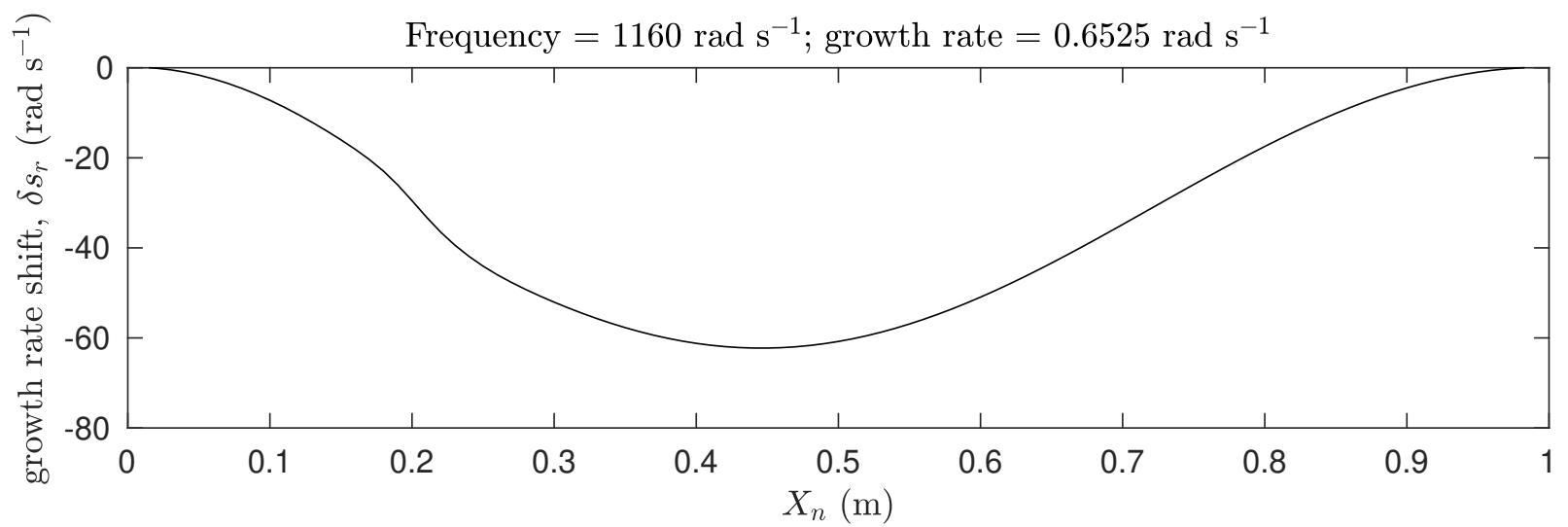

FIG. 14. Growth rate shift of an oscillation at $\omega=1160 \mathrm{rad} \mathrm{s}^{-1}$ due to a Helmholtz Resonator with natural frequency $\omega_{R}=1020 \mathrm{rad} \mathrm{s}^{-1}$, as a function of the resonator position $X_{n}$. 


\section{MATLAB SCRIPTS AND FUNCTIONS}

Run_Helm.m

Read in the dimensional parameters with fun_param_dim.m

Calculate the reference scales and nondimensional parameters with fun_nondim.m

Set the numerical scheme, starting $s$, and number of iterations, $J$

Call fun_Helm.m

Plot the requested output

Perform a Taylor Test if requested.

\begin{tabular}{|c|c|c|}
\hline \multicolumn{3}{|c|}{ Input arguments } \\
\hline name & values/type & description/contents \\
\hline discretization & FDS, FEW, FDW, or SBP & as for fun_Helm.m \\
\hline type_of_adjoint & DA or $\mathrm{CA}$ & as for fun_Helm.m \\
\hline lin_or_nonlin & nonlin or linear & as for fun_Helm.m \\
\hline plot_type & emode, bs_sens, & Output to be plotted: eigenmode, base state \\
\hline (optional) & fb_sens, rec & sensitivities, feedback sensitivities, or receptivities. \\
\hline TT_int (optional) & cell array & as for fun Helm.m \\
\hline TT_ext (optionsl) & cell array & as for fun_Helm.m \\
\hline
\end{tabular}

Example calls from the run line

$\gg$ Run_Helm('FDS', 'CA', 'nonlin')

$\gg$ Run_Helm('FEW', 'DA', 'nonlin', 'emode')

$\gg$ Run_Helm ('FDW', 'CA', 'linear', 'bs_sens')

$\gg$ Run_Helm('SBP', 'DA', 'linear', 'fb_sens', $\{$ 't' $\},\{\}$ )

$\gg$ Run_Helm ('FDW', 'DA', 'linear', 'rec', $\{$ 'all' $\},\{$ 'all' $\}$ ) 


\section{fun_Helm.m}

Read in the gridpoint positions and discretization matrices (FE, FE, or SBP).

Construct matrices $\mathbf{A}$ and $\mathbf{C}$ using the relevant discretization (FDS, FEW, FDW, or SBP) and b.c.'s

Iterate to find the left eigenvector, eigenvalue, and right eigenvector.

Read in the matrices that transform the eigenvectors into receptivities

Wrap the outputs into the emode structure and return if no sensitivities are requested.

Calculate and return the internal sensitivities ds_int

Calculate and return the external sensitivities ds_ext

Perform a Taylor Test if requested.

\begin{tabular}{|c|c|c|}
\hline \multicolumn{3}{|c|}{ Input arguments } \\
\hline name & values/type & description/contents \\
\hline discretization & FDS, FEW, FDW, or SBP & Type of spatial discretization \\
\hline type_of_adjoint & DA or CA, & $\begin{array}{l}\text { Type of adjoint: discrete adjoint or continuous } \\
\text { adjoint }\end{array}$ \\
\hline lin_or_nonlin & nonlin or linear & $\begin{array}{l}\text { Iteration procedure: nonlinear using a Newton } \\
\text { method, or linear using the active iteration method }\end{array}$ \\
\hline param & structure & $\begin{array}{l}\text { The nondimensional parameters: } \gamma, \zeta, n, \tau, X_{w}, L_{w} \text {, } \\
X_{h}, L_{h}, R_{u}, R_{d}, \rho_{u}, \rho_{d}, k_{u} s^{-1}, k_{d} s^{-1}, c_{u}, c_{d} .\end{array}$ \\
\hline scheme & .N.s0 .itmax & $\begin{array}{l}\text { The number of elements, } N \text {, the starting value of } s \text {, } \\
\text { and the number of iterations, } J \text {, for the active } \\
\text { iteration method }\end{array}$ \\
\hline TT_int & cell array & $\begin{array}{l}\text { the base state sensitivities to be tested, e.g. } \\
\left\{\text { t' }^{\prime},{ }^{\prime} \mathrm{h} \text { ' }\right\},\left\{\text { 'all }^{\prime}\right\} \text {, or }\{\}\end{array}$ \\
\hline TT_ext & cell array & $\begin{array}{l}\text { the external sensitivities to be tested, e.g. } \\
\left\{{ }^{\prime} \mathrm{X}_{-} \mathrm{w}^{\prime},{ }^{\prime} \mathrm{R}_{-} \mathrm{u}^{\prime}\right\},\left\{\text { ' }^{\prime} \mathrm{all} \mathrm{I}^{\prime}\right\} \text {, or }\{\}\end{array}$ \\
\hline
\end{tabular}




\begin{tabular}{|c|c|c|}
\hline \multicolumn{3}{|c|}{ Output arguments (Fun_Helm.m) } \\
\hline name & values/type & description/contents \\
\hline emode & structure & $\begin{array}{l}\text { For DA: discretization points, } \mathbf{x} \text {, left eigenvector, } \mathbf{p}^{\dagger} \text {, } \\
\text { eigenvalue, } s \text {, right eigenvector, } \mathbf{p} \text {, discrete receptivities } \\
\dot{m}_{\bar{\rho}}^{\dagger}, \mathbf{f}_{\bar{\rho}}^{\dagger}, \dot{q}_{\bar{p}}^{\dagger} \text {, mass matrix } \mathbf{M} \text {. } \\
\text { For CA: discretization points, } \mathbf{x} \text {, right eigenvector, } \mathbf{p}^{\dagger} \text {, } \\
\text { continuous receptivities } \dot{m}_{\bar{\rho}}^{\dagger}, \mathbf{f}_{\bar{\rho}}^{\dagger}, \dot{q}_{\bar{p}}^{\dagger}\end{array}$ \\
\hline ds_int & structure & $\begin{array}{l}\text { For DA: base state sensitivities }(\partial s / \partial n),\left(\partial s / \partial k_{u}\right), \\
\left(\partial s / \partial k_{d}\right), \text { discrete base state sensitivities }(\partial s / \partial \tau), \\
(\partial s / \partial \mathbf{h}),\left(\partial s / \partial \mathbf{w}_{\bar{\rho}}\right),(\partial s / \partial \overline{\mathbf{v}}), \text { discrete feedback } \\
\text { sensitivities }\left(\partial s / \partial \dot{m}_{\bar{\rho}, u}\right),\left(\partial s / \partial \dot{m}_{\bar{\rho}, p}\right),\left(\partial s / \partial \mathbf{f}_{\bar{\rho}, u}\right) \\
\left(\partial s / \partial \mathbf{f}_{\bar{\rho}, p}\right),\left(\partial s / \partial \dot{q}_{\bar{p}, u}\right),\left(\partial s / \partial \dot{q}_{\bar{p}, p}\right) \\
\text { For CA: base state sensitivities }(\partial s / \partial n),\left(\partial s / \partial k_{u}\right) \\
\left(\partial s / \partial k_{d}\right), \text { continuous base state sensitivities }(\partial s / \partial \tau)_{\text {cont }}, \\
(\partial s / \partial \mathbf{h})_{\text {cont }},\left(\partial s / \partial \mathbf{w}_{\bar{\rho}}\right)_{\text {cont }},(\partial s / \partial \overline{\mathbf{v}})_{\text {cont }}, \text { continuous } \\
\text { feedback sensitivities }\left(\partial s / \partial \dot{m}_{\bar{\rho}, u}\right)_{\text {cont }},\left(\partial s / \partial \dot{m}_{\bar{\rho}, p}\right)_{\text {cont }}, \\
\left(\partial s / \partial \mathbf{f}_{\bar{\rho}, u}\right)_{\text {cont }},\left(\partial s / \partial \mathbf{f}_{\bar{\rho}, p}\right)_{\text {cont }},\left(\partial s / \partial \dot{q}_{\bar{p}, u}\right)_{\text {cont }},\left(\partial s / \partial \dot{q}_{\bar{p}, p}\right)_{\text {cont }},\end{array}$ \\
\hline ds_ext & structure & $\begin{array}{l}\partial s / \partial n, \partial s / \partial \tau, \partial s / \partial X_{w}, \partial s / \partial L_{w}, \partial s / \partial X_{h}, \partial s / \partial L_{h} \\
\partial s / \partial R_{u}, \partial s / \partial R_{d}\end{array}$ \\
\hline
\end{tabular}

\section{mat_FD.m}

Generate the Gauss-Lobatto gridpoints, x, and the differentiation matrix, D Generate the mass matrix $\mathbf{M}$ for the finite difference schemes FDS and FDW. Calculate the density at $\mathbf{x}$ with fun_rh.m and create the density matrix $\mathbf{V}$. Calculate the heat release at $\mathbf{x}$ with fun $\mathrm{h} . \mathrm{m}$.

Calculate the measurement profile and its derivative at $\mathbf{x}$ with fun_wr.m. Generate the time delay vector, $\tau$, at $\mathbf{x}$.

\begin{tabular}{|l|l|l|}
\hline \hline \multicolumn{2}{|l|}{} \\
\hline name & values/type & Input arguments \\
\hline param & structure & as for fun_Helm.m. \\
\hline N & N & The number of elements, $N$. \\
\hline \hline \multicolumn{2}{l|}{} & Output arguments \\
\hline name & values/type & description/contents \\
\hline pos &. $\mathrm{x}$ & The vector $\mathbf{x}$ \\
\hline mat & structure & The matrices $\mathbf{D}, \mathbf{M}, \mathbf{V}$, and the vectors $\tau, \mathbf{h}, \mathbf{w}_{\bar{\rho}}, \mathbf{w}_{\bar{\rho}}^{\prime}$ \\
\hline
\end{tabular}


mat_FE.m

Generate equi-spaced points for P0 and P1 functions, $\mathbf{x}_{0}$ and $\mathbf{x}_{1}$.

Generate the differentiation matrix, $\mathbf{D}_{01}$, and mass matrices $\mathbf{M}_{00}, \mathbf{M}_{01}$, and $\mathbf{M}_{11}$

Calculate the density at $\mathbf{x}_{0}$ with fun_rh.m and create the density matrix $\mathbf{V}_{00}$.

Calculate the heat release at $\mathbf{x}_{1}$ with fun_h.m.

Calculate the measurement profile at $\mathbf{x}_{0}$ with fun_wr.m

Calculate the derivative of the measurement profile at $\mathbf{x}_{1}$.

Generate the time delay vector, $\tau$, at $\mathbf{x}_{1}$.

\begin{tabular}{|c|c|c|}
\hline \multicolumn{3}{|r|}{ Input arguments } \\
\hline name & values/type & description/contents \\
\hline param & structure & as for fun_Helm.m. \\
\hline $\mathrm{N}$ & N & The number of elements, $N$. \\
\hline \multicolumn{3}{|r|}{ Output arguments } \\
\hline name & values/type & description/contents \\
\hline pos & $. \mathrm{x} 0, . \mathrm{x} 1$ & The vectors $\mathbf{x}_{0}$ and $\mathbf{x}_{1}$ \\
\hline mat & structure & $\begin{array}{l}\text { The matrices } \mathbf{D}_{01}, \mathbf{M}_{00}, \mathbf{M}_{01}, \mathbf{M}_{11}, \mathbf{V}_{00} \text {, and the vectors } \\
\mathbf{h}_{1}, \mathbf{w}_{\bar{\rho} 0}, \mathbf{w}_{\bar{\rho} 1}^{\prime}\end{array}$ \\
\hline
\end{tabular}

mat_SBP.m

Generate $N+1$ equi-spaced points, $\mathbf{x}_{1}$, and their mid-points, $\mathbf{x}_{0}$

Generate the first order differentiation matrix, $\mathbf{D}_{1}$, and mass matrices $\mathbf{M}_{00}, \mathbf{M}_{01}$, and $\mathbf{M}$

Generate the first derivative operator at the boundaries, $\mathbf{S}$

Generate a building block matrix for the second derivative operator: $\mathbf{D}_{01}$

Generate building block matrices for the simultaneous approximation term: $\mathbf{E}_{d}, \mathbf{E}_{u}, \mathbf{I}$

Calculate the density at $\mathbf{x}_{0}$ and $\mathbf{x}_{1}$ with fun_rh.m and create the density matrices $\mathbf{V}_{00}$ and $\mathbf{V}_{11}$

Calculate the heat release at $\mathbf{x}_{1}$ with fun_h.m

Calculate the measurement profile at $\mathbf{x}_{1}$ with fun_wr.m

Calculate the derivative of the measurement profile at $\mathbf{x}_{1}$.

Generate the time delay vector, $\tau$, at $\mathbf{x}_{1}$.

\begin{tabular}{|c|c|c|}
\hline \multicolumn{3}{|r|}{ Input arguments } \\
\hline name & values/type & description/contents \\
\hline param & structure & as for fun_Helm.m. \\
\hline $\mathrm{N}$ & $\mathrm{N}$ & The number of elements, $N$. \\
\hline \multicolumn{3}{|r|}{ Output arguments } \\
\hline name & values/type & description/contents \\
\hline pos & $. \mathrm{x} 0, . \mathrm{x} 1$ & The vectors $\mathbf{x}_{0}$ and $\mathbf{x}_{1}$. \\
\hline mat & structure & $\begin{array}{l}\text { The matrices } \mathbf{D}_{1}, \mathbf{D}_{01}, \mathbf{M}, \mathbf{M}_{00}, \mathbf{M}_{01}, \mathbf{V}_{00}, \mathbf{V}_{11}, \mathbf{S}, \mathbf{E}_{d}, \\
\mathbf{E}_{u}, \mathbf{I}, \text { and the vectors } \mathbf{h}, \mathbf{w}_{\bar{\rho}}, \mathbf{w}_{\bar{\rho}}^{\prime}\end{array}$ \\
\hline
\end{tabular}


mat_AC_**_DA.m

Construct the $\mathbf{A}, \mathbf{C}$, and $\mathrm{d} \mathbf{A} / \mathrm{d} s$ matrices for the direct equations in FDS (13), FEW (15), FDW (16), SBP $(17)$

Apply boundary conditions with fun_bcs_strong.m or fun_bcs_weak.m.

Generate the matrices that map from $\mathbf{p}$ to $\mathbf{u}$ and from $\mathbf{p}^{\dagger}$ to the receptivities $\dot{m}_{\bar{\rho}}^{\dagger}, \mathbf{F}_{\bar{\rho}}^{\dagger}$, and $\dot{q}_{\bar{p}}^{\dagger}$.

Generate $(\mathrm{d} \mathbf{A} / \mathrm{d} b)_{l}$ and $(\mathrm{d} \mathbf{A} / \mathrm{d} b)_{r}$ for all the internal parameters, $b$.

(These are the terms in Table VII before they have been multiplied by $\mathbf{p}^{\dagger}$ and $\mathbf{p}$.)

Apply boundary conditions to $(\mathrm{d} \mathbf{A} / \mathrm{d} b)_{l}$ and $(\mathrm{d} \mathbf{A} / \mathrm{d} b)_{r}$.

\begin{tabular}{|c|c|c|}
\hline \multicolumn{3}{|r|}{ Input arguments } \\
\hline name & values/type & description/contents \\
\hline mat & structure & as for mat_FD.m, mat_FE.m, or mat_SBP.m \\
\hline param & structure & as for fun_Helm.m. \\
\hline $\mathrm{N}$ & $\mathrm{N}$ & The number of elements, $N$. \\
\hline $\mathrm{s}$ & $\mathrm{S}$ & The eigenvalue, $s$. \\
\hline \multicolumn{3}{|r|}{ Output arguments } \\
\hline name & values/type & description/contents \\
\hline A & matrix & The matrix $\mathbf{A}$ with boundary conditions applied \\
\hline C & matrix & The matrix $\mathbf{C}$ with boundary conditions applied \\
\hline dAds & matrix & The matrix $\mathrm{dA} / \mathrm{d} s$ with boundary conditions applied \\
\hline $\mathrm{T}$ & structure & matrices that convert $\mathbf{p}$ and $\mathbf{p}^{\dagger}$ into $\mathbf{u}$ and the receptivities \\
\hline $\mathrm{dA}$ & structure & matrices and vectors $(\mathrm{d} \mathbf{A} / \mathrm{d} b)_{l}$ and $(\mathrm{d} \mathbf{A} / \mathrm{d} b)_{r}$ \\
\hline ds_int & structure & The structure in fun_Helm.m with all values set to zero \\
\hline
\end{tabular}

mat_AC_**_CA.m

Construct A, C, and dA/d $s$ matrices for the continuous adjoint in FDS (27), FEW (28), FDW (29), SBP (30).

Apply boundary conditions with fun_bcs_strong.m or fun_bcs_weak.m.

Generate the matrices that convert from $\mathbf{p}^{\dagger}$ to the receptivities $\dot{m}_{\bar{\rho}}^{\dagger}, \mathbf{F}_{\bar{\rho}}^{\dagger}$, and $\dot{q}_{\bar{p}}^{\dagger}$.

\begin{tabular}{|c|c|c|}
\hline \multicolumn{3}{|r|}{ Input arguments } \\
\hline name & values/type & description/contents \\
\hline mat & structure & as for mat_FD.m, mat_FE.m, or mat_SBP.m \\
\hline param & structure & as for fun_Helm.m. \\
\hline $\mathrm{N}$ & $\mathrm{N}$ & The number of elements, $N$. \\
\hline conjs & conjs & The complex conjugate of the eigenvalue, $s$. \\
\hline \multicolumn{3}{|r|}{ Output arguments } \\
\hline name & values/type & description/contents \\
\hline A & matrix & The matrix $\mathbf{A}$ with boundary conditions applied \\
\hline C & matrix & The matrix $\mathbf{C}$ with boundary conditions applied \\
\hline dAds & matrix & The matrix $\mathrm{d} \mathbf{A} / \mathrm{d} s$ with boundary conditions applied \\
\hline $\mathrm{T}$ & structure & matrices that convert $\mathbf{p}^{\dagger}$ into the receptivities \\
\hline
\end{tabular}




\begin{tabular}{|l|l|}
\hline \multicolumn{2}{|c|}{ Remaining functions } \\
\hline \hline name & description \\
\hline fun_bcs_strong.m & Apply the boundary conditions to the strong form equations \\
\hline fun_bcs_weak.m & Apply the boundary conditions to the weak form equations \\
\hline fun_ds_CA.m & Evaluate the internal sensitivities in the continuous adjoint framework \\
\hline fun_ds_DA.m & Evaluate the internal sensitivities in the discrete adjoint framework \\
\hline fun_eig_nearest.m & Solve $\mathbf{A p}=s^{2} \mathbf{C}$ p and select the eigenmode with $s$ closest to $s_{0}$. \\
\hline fun_ext_int_CA.m & Evaluate the external sensitivities in the continuous adjoint framework. \\
\hline fun_ext_int_DA.m & Evaluate the external sensitivities in the discrete adjoint framework. \\
\hline fun_h.m & Set the heat release envelope, h, and its partial derivatives. \\
\hline fun_kukd.m & Calculate the Robin boundary coefficients, $k_{u}$ and $k_{d}$. \\
\hline fun_nondim.m & Convert the dimensional parameters to nondimensional parameters. \\
\hline fun_normalize.m & normalize p such that $\mathbf{p}^{T} \mathbf{M p}=1$. \\
\hline fun_param_dim.m & Set the dimensional parameters. \\
\hline fun_rh.m & Set the density profile and its partial derivatives. \\
\hline fun_set_s0.m & Use a travelling wave method to estimate $s_{0}$ for the requested mode. \\
\hline fun_TT.m & Perform a Taylor Test on the fields held in TT_int and TT_ext. \\
\hline fun_wr.m & Set the measurement envelope, w, and its partial derivatives. \\
\hline unwrap_*.m & Unwrap variables from structure $*$. \\
\hline wrap_*.m & Wrap variables into structure $*$. \\
\hline
\end{tabular}


[1] F. Nicoud, L. Benoit, C. Sensiau, and T. Poinsot. Acoustic Modes in Combustors with Complex Impedances and Multidimensional Active Flames. AIAA Journal, 45(2):426-441, 2007.

[2] T. C. Lieuwen and V. Yang. Combustion Instabilities in Gas Turbine Engines: Operational Experience, Fundamental Mechanisms, and Modeling. AIAA, 2005.

[3] J. W. S. B. Rayleigh. The Explanation of Certain Acoustical Phenomena. Nature, 18(455):319$321,1878$.

[4] B.-T. Chu. On the energy transfer to small disturbances in fluid flow (Part I). Acta Mechanica, 1(3):215-234, 1965.

[5] M. P. Juniper and R. I. Sujith. Sensitivity and Nonlinearity of Thermoacoustic Oscillations. Annual Review of Fluid Mechanics, 50:661-689, 2018.

[6] T. C. Lieuwen and K. R. McManus. Combustion Dynamics in Lean-Premixed Prevaporized (LPP) Gas Turbines. Journal of Propulsion and Power, 19(5):721, 2003.

[7] H. C. Mongia, T. J. Held, G. C. Hsiao, and R. P. Pandalai. Challenges and Progress in Controlling Dynamics in Gas Turbine Combustors Introduction. Journal of Propulsion and Power, 19(5):822-829, 2003.

[8] Y. Huang and V. Yang. Dynamics and stability of lean-premixed swirl-stabilized combustion. Progress in Energy and Combustion Science, 35(4):293-364, 2009.

[9] G. Richards, D. L. Strauss, and E. H. Robey. Passive Control of Combustion Dynamics in Stationary Gas Turbines. Journal of Propulsion and Power, 19(5):795-810, 2003.

[10] L. Crocco and S.-I. Cheng. Theory of Combustion Instability in Liquid Propellant Rocket Motors. Butterworths, London, 1956.

[11] P. Luchini and A. Bottaro. Adjoint Equations in Stability Analysis. Annual Review of Fluid Mechanics, 46(1):493-517, 2014.

[12] L. Magri and M. P. Juniper. Sensitivity analysis of a time-delayed thermo-acoustic system via an adjoint-based approach. Journal of Fluid Mechanics, 719:183-202, 2013.

[13] L. Magri and M. P. Juniper. Global modes, receptivity, and sensitivity analysis of diffusion flames coupled with duct acoustics. Journal of Fluid Mechanics, 752:237-265, 2014. 
[14] T. Sayadi, V. Le Chenadec, P. J. Schmid, F. Richecoeur, and M. Massot. Thermoacoustic instability - a dynamical system and time domain analysis. Journal of Fluid Mechanics, 753:448-471, 2014.

[15] J. G. Aguilar, L. Magri, and M. P. Juniper. Adjoint-based sensitivity analysis of low-order thermoacoustic networks using a wave-based approach. Journal of Computational Physics, 341:163-181, 2017.

[16] J. G. Aguilar and M. P. Juniper. Shape optimization in low-order thermoacoustic networks. In Proceedings of GPPS Forum 18, number 0024, 2018.

[17] J. G. Aguilar and M. P. Juniper. Adjoint methods for elimination of thermoacoustic oscillations in a model annular combustor via small geometry modifications. In ASME Turbo Expo, page $75692,2018$.

[18] S. R. Stow and A. P. Dowling. Thermoacoustic oscillations in an annular combustor. In ASME Turbo Expo, 2001-GT-0037, New Orleans, 2001. ASME.

[19] S. R. Stow and A. P. Dowling. Low order modelling of thermoacoustic limit cycles. In ASME Turbo Expo, GT2004-54245, Vienna, 2004. ASME.

[20] M. P. Juniper, L. Magri, M. Bauerheim and F. Nicoud. Sensitivity analysis of thermo-acoustic eigenproblems with adjoint methods. In Centre for Turbulence Research, Proceedings of the Summer Program, 2014.

[21] G. A. Mensah and J. P. Moeck. Acoustic Damper Placement and Tuning for Annular Combustors: an Adjoint-Based Optimization Study. Journal of Engineering for Gas Turbines and Power, 139:061501, 2017.

[22] G. A. Mensah, L. Magri, and J. P. Moeck. Methods for the Calculation of Thermoacoustic Stability Boundaries and Monte Carlo-Free Uncertainty Quantification. Journal of Engineering for Gas Turbines and Power, 140:1-10, 2018.

[23] C. F. Silva, L. Magri, T. Runte, and W. Polifke. Uncertainty Quantification of Growth Rates of Thermoacoustic Instability by an Adjoint Helmholtz Solver. Journal of Engineering for Gas Turbines and Power, 139:011901, 2018.

[24] G. A. Mensah, L. Magri, A. Orchini and J. P. Moeck. Effects of asymmetry on thermoacoustic modes in annular combustors: a higher order perturbation study. In ASME Turbo Expo, GT2018-76797, 2018. 
[25] A. Ndiaye. Quantification des incertitudes pour la prédiction des instabilités thermoacoustiques dans les chambres de combustion. PhD thesis, Université de Montpellier, 2017.

[26] L. N.ZTrefethen. Spectral Methods in Matlab. SIAM, Philadelphia, 2000.

[27] R. Kamakoti and C. Pantano. High-Order Narrow Stencil Finite-Difference Approximations of Second-Order Derivatives involving Variable Coefficients. SIAM Journal of Scientific Computing, 31(6):4222-4243, 2009.

[28] J. E. Hicken and D. W. Zingg. Superconvergent Functional Estimates form Summation-byParts Finite-Difference Discretizations. SIAM Journal of Scientific Computing, 33(2):893-922, 2011.

[29] J. Berg and J. Nordström. Duality based boundary conditions and dual consistent finite difference discretizations of the Navier-Stokes and Euler equations. Journal of Computational Physics, 259:135-153, 2014.

[30] S. Eriksson. A Dual Consistent Finite Difference Method with Narrow Stencil Second Derivative Operators. Journal of Scientific Computing, 75(2):906-940, 2018.

[31] K. Mattsson and J. Nordström. Summation by parts operators for finite difference approximations of second derivatives. Journal of Computational Physics, 199:503-540, 2004.

[32] T. Nystrand. Summation by Part Methods for Poisson's Equation with Discontinuous Variable Coefficients. PhD thesis, Uppsala Universitet, 2014.

[33] G. Rigas, N. P. Jamieson, L. K. B. Li, and M. P. Juniper. Experimental sensitivity analysis and control of thermoacoustic systems. Journal of Fluid Mechanics, 787:R1 1-11, 2016.

[34] G. J. Chandler, M. P. Juniper, J. W. Nichols, and P. J. Schmid. Adjoint algorithms for the Navier-Stokes equations in the low Mach number limit. Journal of Computational Physics, 231:1900-1916, 2012.

[35] L. Magri, M. Bauerheim, and M. P. Juniper. Stability analysis of thermo-acoustic nonlinear eigenproblems in annular combustors. Part I Sensitivity. Journal of Computational Physics, 325:395-410, 2016.

[36] J.-M. Chomaz. Global Instabilities in Spatially Developing Flows: Non-Normality and Nonlinearity. Annual Review of Fluid Mechanics, 37(1):357-392, 2005.

[37] D. Sipp, O. Marquet, P. Meliga and A. Barbagallo. Dynamics and Control of Global Instabilities in Open-Flows: A Linearized Approach. Applied Mechanics Reviews, 63:030801, 2010. 
[38] M. P. Juniper and L. Magri. Application of receptivity and sensitivity analysis to thermoacoustic instability. In Von Karman Institute course notes, June, 2014.

[39] L. Magri, M. Bauerheim, F. Nicoud and M. P. Juniper. Stability analysis of thermo-acoustic nonlinear eigenproblems in annular combustors. Part II Uncertainty quantification. Journal of Computational Physics, 325:411-421, 2016.

[40] D. S. Miller. Internal Flow Systems. 1978.

[41] N. P. Jamieson and M. P. Juniper. Experimental sensitivity analysis of a linearly stable thermoacoustic system via a pulsed forcing technique. Experiments in Fluids, 58(9):1-12, 2017.

[42] J. Aguilar. Sensitivity analysis and optimization in low order thermoacoustic models. PhD thesis, University of Cambridge, 2018.

[43] S. W. Rienstra and A. Hirschberg. An Introduction to Acoustics. 2006.

[44] D. Funaro and D. Gottlieb. A New Method of Imposing Boundary Conditions in Pseudospectral Approximations of Hyperbolic Equations. Mathematics of Computation, 51(184):599-613, 1988.

[45] D. C. Del Rey Fernández, J. E. Hicken, and D. W. Zingg. Review of summation-by-parts operators with simultaneous approximation terms for the numerical solution of partial differential equations. Computers \& Fluids, 95:171-196, 2014. 